\title{
inorganics
}

ISSN 2304-6740

www.mdpi.com/journal/inorganics

Review

\section{Molecular Pnictogen Activation by Rare Earth and Actinide Complexes}

\section{Zoë R. Turner}

Chemistry Research Laboratory, Department of Chemistry, University of Oxford, Mansfield Road, Oxford OX13TA, UK; E-Mail: zoe.turner@chem.ox.ac.uk; Tel.: +44-1865-285-157

Academic Editors: Stephen Mansell and Steve Liddle

Received: 1 October 2015 / Accepted: 9 December 2015 / Published: 21 December 2015

\begin{abstract}
This review covers the activation of molecular pnictogens (group 15 elements) by homogeneous rare earth and actinide complexes. All examples of molecular pnictogen activation (dinitrogen, white phosphorus, yellow arsenic) by both rare earths and actinides, to date (2015), are discussed, focusing on synthetic methodology and the structure and bonding of the resulting complexes.
\end{abstract}

Keywords: group 3; rare earth; lanthanide; actinide; dinitrogen; white phosphorus; yellow arsenic; pnictogen; small molecule activation

\section{Introduction}

Rare earth (scandium, yttrium and the lanthanides) and actinide complexes remain underexplored with respect to the transition metals and main group elements but often demonstrate both unique reactivity and molecular properties. Understanding of the bonding and electronic structure of these complexes has particular significance for separation of metals in nuclear waste streams [1].

Activation of molecular pnictogens (group 15 elements) is an area of growing importance; atmospheric dinitrogen $\left(\mathrm{N}_{2}\right)$ and white phosphorus $\left(\mathrm{P}_{4}\right)$ are principal sources of $\mathrm{N}$ - and $\mathrm{P}$-containing compounds (e.g., polymers, pharmaceuticals, agrochemicals, explosives, and specialty chemicals) but are both very challenging to selectively activate. Metal-arsenic, -antimony and -bismuth complexes remain rare [2-4], while the study of metal-pnictogen complexes, including these heavier pnictogen homologues, is also of fundamental importance with respect to Ln and An-pnictogen bonding and electronic structure. 
Fixation of $\mathrm{N}_{2}$, the six electron reduction to two molecules of more reactive ammonia, is necessary for further formation of $\mathrm{N}$-element bonds. In nature, nitrogenase enzymes containing metalloproteins (Fe, Mo or $\mathrm{V}$ ) fix $\mathrm{N}_{2}$ through proton-coupled electron transfer under ambient conditions [5,6]. In industry, the Haber-Bosch process combines $\mathrm{N}_{2}$ and high purity $\mathrm{H}_{2}$ at high temperatures and pressures over heterogeneous iron- or ruthenium-based catalysts [7-10]. This highly efficient process produces 100 million tons of ammonia per year but is the largest energy-consuming process in the modern world today; the need for direct activation and functionalisation of $\mathrm{N}_{2}$ under mild conditions is a clear goal. Accessing appropriately reactive phosphorus building blocks presents a different set of challenges based on the sustainability and efficiency of chemical transformations required; from phosphate rock minerals which are mined globally on a 225 million ton scale per year (2013) [11], phosphate fertilisers derived from phosphoric acid are the major products with the remainder used for elemental phosphorus production. Organophosphorus compounds are generally derived from $\mathrm{PCl}_{3}$, obtained by the chlorination of $\mathrm{P}_{4}$, and subsequent multi-step procedures [12-14]. Attention has turned to direct and selective activation of elemental phosphorus under mild conditions; this approach is more atom-efficient (which is important given the limited accessible deposits of phosphate rock), avoids the need for large scale production of $\mathrm{PCl}_{3}$ (which is toxic, corrosive and highly reactive), and is both more economically and environmentally sustainable [15].

The area of dinitrogen activation has been reviewed extensively with particular focus on the HaberBosch process [16-18], and biological nitrogen fixation [5,6,19-26]. There are reviews on transition metal $\mathrm{N}_{2}$ activation which cover $\mathrm{N}_{2}$ binding modes [27-29], multimetallic $\mathrm{N}_{2}$ activation [30,31], the relevance of metal hydride complexes to $\mathrm{N}_{2}$ activation [32,33], $\mathrm{N}_{2}$ cleavage and functionalisation [34,35] (including electrochemical [36] and photolytic $\mathrm{N}_{2}$ cleavage [37]), and $\mathrm{N}_{2}$ activation at bare metal atoms [38] and using surface organometallic chemistry [39]. Specific reviews have also focused on activation by group 4 metals [40-42], iron [31,43,44], molybdenum [24,45-47], and the mid-to-late transition metal centres [48]. In terms of rare earth $\mathrm{N}_{2}$ activation; an account of work from Evans and co-workers to 2004 has been reported [49], and Gardiner more recently reviewed the chemistry of the lanthanides with dinitrogen and reduced derivatives [50]. The area of actinide $\mathrm{N}_{2}$ activation has been discussed in the context of small molecule activation by trivalent uranium complexes $[51,52]$.

Transition metal-mediated white phosphorus activation has been previously reviewed [53-56], with specific reviews on both early transition metal complexes [57,58] and late transition metal complexes [59]. More broadly, reviews of $\mathrm{P}_{4}$ activation by $p$-block compounds have also been reported [60-64].

This review seeks to cover all examples of molecular pnictogen activation (dinitrogen, white phosphorus, yellow arsenic) by both rare earth and actinide complexes to date, focusing on synthetic methodology and the structure and bonding of the resulting complexes. Only well-defined homogeneous complexes will be discussed; heterogeneous and surface chemistry lie beyond the scope of this review.

\section{Dinitrogen Activation by Rare Earth Complexes}

\subsection{Complexes Containing a Formal $\mathrm{N}_{2}{ }^{2-}$ Ligand}

The majority of rare earth complexes that activate dinitrogen $\left(\mathrm{N}_{2}\right)$ result in its formal reduction to the $\mathrm{N}_{2}{ }^{2-}$ anion and the formation of bimetallic complexes of the general form $\left[\mathrm{A}_{2}(\operatorname{thf})_{x} \mathrm{Ln}\right]_{2}\left(\mu-\eta^{2}: \eta^{2}-\mathrm{N}_{2}\right)$ 
where there is side-on binding of $\mathrm{N}_{2}$ (" $\mathrm{A}$ is defined as a group that exists as an anion in $\mathrm{LnA}_{3}$ and provides reductive reactivity in combination with an alkali metal or the equivalent") [65]. The work of Evans and co-workers has led to the development of three key methodologies to access these species: (i) salt metathesis reactions of divalent lanthanide halides with alkali metal salts; (ii) combination of trivalent $\mathrm{Ln}$ complexes with alkali metals ( $\mathrm{LnA}_{3} / \mathrm{M}$ or $\mathrm{LnA}_{2} \mathrm{~A}^{\prime} / \mathrm{M}$ method); (iii) photochemical activation of $\mathrm{LnA}_{2} \mathrm{~A}^{\prime}$ systems (Figure 1).

From divalent Ln

Direct $\mathrm{N}_{2}$ reduction

$$
\begin{aligned}
2 \mathrm{LnI}_{2}+ & 4 \mathrm{MA} \frac{\mathrm{N}_{2}}{-4 \mathrm{Ml}} \\
\mathrm{Ln}= & \mathrm{Nd}, \mathrm{Sm}, \mathrm{Dy}, \mathrm{Tm} \\
\mathrm{MA}= & \mathrm{KCp}{ }^{\mathrm{R}} \\
& \mathrm{KN}\left(\mathrm{SiMe}_{3}\right)_{2} \\
& \mathrm{KO}-2,6-{ }^{\mathrm{B}} \mathrm{Bu}-\mathrm{C}_{6} \mathrm{H}_{3}
\end{aligned}
$$

\section{From trivalent Ln}

Photochemical activation

$\operatorname{LnA}_{3} / M$ or $\operatorname{Ln} A_{2} A^{\prime} / M$

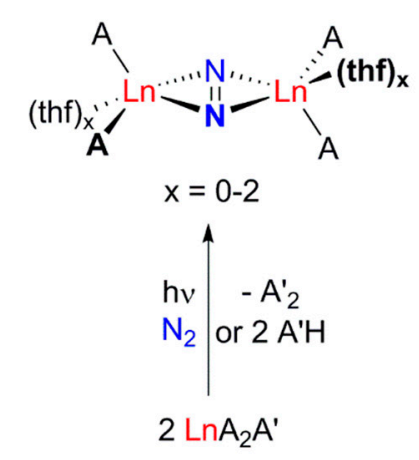

$\operatorname{Ln}=Y, L u, D y$

$\mathrm{A}=\eta^{5}-\mathrm{C}_{5} \mathrm{Me}_{5},-\mathrm{C}_{5} \mathrm{Me}_{4} \mathrm{H}$

$A^{\prime}=\eta^{3}-\mathrm{C}_{5} \mathrm{Me}_{4} \mathrm{H},-\mathrm{C}_{3} \mathrm{H}_{5},-2-\mathrm{Me}-\mathrm{C}_{3} \mathrm{H}_{4}$

$$
\begin{aligned}
& \frac{\mathrm{N}_{2}}{-2 \mathrm{MA}} 2 \mathrm{LnA}_{3}+2 \mathrm{M} \\
& \frac{\mathrm{N}_{2}}{-2 \mathrm{MA}^{\prime}} \quad 2 \mathrm{LnA}_{2} \mathrm{~A}^{\prime}+2 \mathrm{M} \\
& \text { Ln = Sc, Y, La, Ce, Pr, Nd, Gd } \\
& \text { Tb, Dy, Ho, Er, Tm, Lu } \\
& \mathrm{M}=\mathrm{K}, \mathrm{KC}_{8}, \mathrm{Na} \\
& \mathrm{A}=\mathrm{KC}_{5} \mathrm{Me}_{5}, \mathrm{KC}_{5} \mathrm{Me}_{4} \mathrm{H}, \mathrm{KC}_{5} \mathrm{H}_{2}{ }^{\mathrm{t}} \mathrm{Bu}_{3} \\
& \mathrm{KC}_{5} \mathrm{H}_{4} \mathrm{SiMe}_{3}, \mathrm{KC}_{5} \mathrm{H}_{3}\left(\mathrm{SiMe}_{3}\right)_{2} \\
& \mathrm{KN}\left(\mathrm{SiMe}_{3}\right)_{2} \\
& \mathrm{KO}-2,6-{ }^{\mathrm{t}} \mathrm{Bu}-\mathrm{C}_{6} \mathrm{H}_{3} \\
& \mathrm{~A}^{\prime}=\mathrm{BPh}_{4}, \mathrm{BH}_{4}, \mathrm{H}, \mathrm{I}
\end{aligned}
$$

\begin{tabular}{|c|c|c|c|c|}
\hline Complex (\#) [Reference] & $\begin{array}{l}\text { N-N Bond } \\
\text { Length }(\AA)\end{array}$ & $\begin{array}{c}\left.\text { Ln-N(N } \mathbf{N}_{2}\right) \\
\text { Bond } \\
\text { Lengths }(\AA ̊)\end{array}$ & $\begin{array}{c}\mathrm{N}-\mathrm{N} \\
\text { Frequency } \\
\left(\mathrm{cm}^{-1}\right)\end{array}$ & $\begin{array}{c}{ }^{14 / 15} \mathrm{~N}-\mathrm{NMR} \\
\text { Spectroscopy } \\
(\mathrm{ppm})^{\mathrm{a}}\end{array}$ \\
\hline $\mathrm{N}_{2}$ & $1.0975[66]$ & - & $2331[67]$ & $-75[68]$ \\
\hline$\left[\left(\eta^{5}-\mathrm{C}_{5} \mathrm{Me}_{4} \mathrm{H}\right)_{2} \mathrm{Sc}\right]_{2}\left(\mu-\eta^{2}: \eta^{2}-\mathrm{N}_{2}\right)(\mathbf{1})[69]$ & $1.239(3)$ & $\begin{array}{l}2.216(1) \\
2.220(1) \\
\end{array}$ & - & - \\
\hline$\left[\left(\eta^{5}-\mathrm{C}_{5} \mathrm{Me}_{4} \mathrm{H}\right)_{2} \mathrm{Sc}\right]_{2}\left(\mu-\eta^{2}: \eta^{2}-\mathrm{N}_{2}\right)\left(\mathbf{1}^{\prime}\right)[70]$ & $1.229(3)$ & $\begin{array}{l}2.197(2) \\
2.179(2) \\
\end{array}$ & - & 385 \\
\hline$\left[\left(\eta^{5}-\mathrm{C}_{5} \mathrm{Me}_{5}\right)_{2} \mathrm{Y}\right]_{2}\left(\mu-\eta^{2}: \eta^{2}-\mathrm{N}_{2}\right)(2)[71]$ & $1.172(6)$ & $\begin{array}{l}2.279(3) \\
2.292(3) \\
\end{array}$ & - & 496 \\
\hline$\left[\left(\eta^{5}-1,2,4-{ }^{t} \mathrm{Bu}-\mathrm{C}_{5} \mathrm{H}_{2}\right)_{2} \mathrm{Nd}\right]_{2}\left(\mu-\eta^{2}: \eta^{2}-\mathrm{N}_{2}\right)(3)[67]$ & $1.226(12)$ & $\begin{array}{l}2.495(2) \\
2.497(2)\end{array}$ & $\begin{array}{l}1622\left({ }^{14} \mathrm{~N}_{2}\right) \\
1569\left({ }^{15} \mathrm{~N}_{2}\right)\end{array}$ & - \\
\hline$\left[\left(\eta^{5}-\mathrm{C}_{5} \mathrm{Me}_{5}\right)_{2} \mathrm{Sm}\right]_{2}\left(\mu-\eta^{2}: \eta^{2}-\mathrm{N}_{2}\right)^{\mathrm{b}}(4)[72]$ & $1.088(12)$ & $\begin{array}{l}2.348(6) \\
2.367(6)\end{array}$ & - & $\begin{array}{l}-117(263 \mathrm{~K}) \\
-161(203 \mathrm{~K})\end{array}$ \\
\hline
\end{tabular}

Figure 1. Routes to $\mathrm{N}_{2}{ }^{2-}$ complexes using rare earth metals.

For reference, $\mathrm{N}-\mathrm{N}$ and $\mathrm{M}-\mathrm{N}\left(\mathrm{N}_{2}\right)$ bond lengths obtained from single crystal $\mathrm{X}$-ray diffraction experiments, N-N stretching frequencies (obtained by IR or Raman spectroscopy) and ${ }^{14 / 15} \mathrm{~N}-\mathrm{NMR}$ spectroscopic data are summarised in Table 1.

Table 1. Summary of rare earth $\mathrm{N}_{2}{ }^{2-}$ complexes. 
Table 1. Cont.

\begin{tabular}{|c|c|c|c|c|}
\hline Complex (\#) [Reference] & $\begin{array}{l}\text { N-N Bond } \\
\text { Length }(\AA)\end{array}$ & 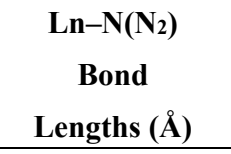 & $\begin{array}{c}\mathrm{N}-\mathrm{N} \\
\text { Frequency } \\
\left(\mathrm{cm}^{-1}\right)\end{array}$ & $\begin{array}{c}{ }^{14 / 15} \mathrm{~N}-\mathrm{NMR} \\
\text { Spectroscopy } \\
(\mathrm{ppm})^{\mathrm{a}}\end{array}$ \\
\hline$\left[\left(\eta^{5}-\mathrm{C}_{5} \mathrm{Me}_{5}\right)_{2} \mathrm{Dy}\right]_{2}\left(\mu-\eta^{2}: \eta^{2}-\mathrm{N}_{2}\right)(\mathbf{5})[73]$ & - & - & - & - \\
\hline$\left[\left(\eta^{5}-\mathrm{SiMe}_{3}-\mathrm{C}_{5} \mathrm{H}_{4}\right)_{2} \mathrm{Dy}\right]_{2}\left(\mu-\eta^{2}: \eta^{2}-\mathrm{N}_{2}\right)(\mathbf{6})[74]$ & & Connectivity only & & - \\
\hline$\left[\left(\eta^{5}-\mathrm{C}_{5} \mathrm{Me}_{5}\right)_{2} \mathrm{Tm}\right]_{2}\left(\mu-\eta^{2}: \eta^{2}-\mathrm{N}_{2}\right)(7)[75]$ & & Connectivity only & & - \\
\hline$\left[\left(\eta^{5}-1,3-\mathrm{SiMe}_{3}-\mathrm{C}_{5} \mathrm{H}_{3}\right)_{2} \mathrm{Tm}\right]_{2}\left(\mu-\eta^{2}: \eta^{2}-\mathrm{N}_{2}\right)(\mathbf{8})[75]$ & $1.259(4)$ & $\begin{array}{l}2.273(2) \\
2.272(2) \\
\end{array}$ & - & - \\
\hline$\left.\left[\left(\eta^{5}-\mathrm{C}_{5} \mathrm{Me}\right)_{2}\right)_{2} \mathrm{Lu}\right]_{2}\left(\mu-\eta^{2}: \eta^{2}-\mathrm{N}_{2}\right)(\mathbf{9})[71]$ & & Connectivity only & & 527 \\
\hline$\left[\left(\eta^{5}-\mathrm{C}_{5} \mathrm{Me}_{5}\right)\left(\eta^{5}-\mathrm{C}_{5} \mathrm{Me}_{4} \mathrm{H}\right) \mathrm{Lu}\right]_{2}\left(\mu-\eta^{2}: \eta^{2}-\mathrm{N}_{2}\right)(\mathbf{1 0})[76]$ & $1.275(3)$ & $\begin{array}{l}2.291(3) \\
2.295(3) \\
\end{array}$ & $\begin{array}{l}1736\left({ }^{14} \mathrm{~N}_{2}\right) \\
1678\left({ }^{15} \mathrm{~N}_{2}\right) \\
\end{array}$ & - \\
\hline$\left[\left(\eta^{5}-\mathrm{C}_{5} \mathrm{Me}_{4} \mathrm{H}\right)_{2} \mathrm{Y}(\text { thf })\right]_{2}\left(\mu-\eta^{2}: \eta^{2}-\mathrm{N}_{2}\right)^{\mathrm{c}}(\mathbf{1 1})[77]$ & $1.252(5)$ & $\begin{array}{l}2.338(3) \\
2.370(3) \\
\end{array}$ & - & 468 \\
\hline$\left[\left(\eta^{5}-\mathrm{SiMe}_{3}-\mathrm{C}_{5} \mathrm{H}_{4}\right)_{2} \mathrm{Y}(\mathrm{thf})\right]_{2}\left(\mu-\eta^{2}: \eta^{2}-\mathrm{N}_{2}\right)^{\mathrm{c}}(\mathbf{1 2})[78]$ & $1.244(2)$ & $\begin{array}{l}2.3214(14) \\
2.3070(14) \\
\end{array}$ & - & - \\
\hline$\left[\left(\eta^{5}-\mathrm{C}_{5} \mathrm{Me}_{5}\right)_{2} \mathrm{La}(\mathrm{thf})\right]_{2}\left(\mu-\eta^{2}: \eta^{2}-\mathrm{N}_{2}\right){ }^{\mathrm{d}}(\mathbf{1 3})[79]$ & $1.233(5)$ & $\begin{array}{l}2.537(4) \\
2.478(4) \\
\end{array}$ & - & 569 \\
\hline$\left[\left(\eta^{5}-\mathrm{C}_{5} \mathrm{Me}_{4} \mathrm{H}\right)_{2} \mathrm{La}(\text { thf })\right]_{2}\left(\mu-\eta^{2}: \eta^{2}-\mathrm{N}_{2}\right)^{\mathrm{c}}(\mathbf{1 4})[79]$ & $1.243(4)$ & $\begin{array}{l}2.457(2) \\
2.503(2) \\
\end{array}$ & - & 495 \\
\hline$\left[\left(\eta^{5}-\mathrm{C}_{5} \mathrm{Me}_{5}\right)_{2} \mathrm{Ce}(\text { thf })\right]_{2}\left(\mu-\eta^{2}: \eta^{2}-\mathrm{N}_{2}\right)^{\mathrm{c}}(\mathbf{1 5})[68]$ & $1.258(9)$ & $\begin{array}{c}2.4548(15) \\
2.542(2) \\
\end{array}$ & - & 871 \\
\hline$\left[\left(\eta^{5}-\mathrm{C}_{5} \mathrm{Me}_{4} \mathrm{H}\right)_{2} \mathrm{Ce}(\mathrm{thf})\right]_{2}\left(\mu-\eta^{2}: \eta^{2}-\mathrm{N}_{2}\right)^{\mathrm{d}}(\mathbf{1 6})[68]$ & $1.235(6)$ & $\begin{array}{l}2.428(3) \\
2.475(3)\end{array}$ & - & 1001 \\
\hline$\left[\left(\eta^{5}-\mathrm{C}_{5} \mathrm{Me}_{5}\right)_{2} \operatorname{Pr}(\mathrm{thf})\right]_{2}\left(\mu-\eta^{2}: \eta^{2}-\mathrm{N}_{2}\right)^{\mathrm{d}}(\mathbf{1 7})[68]$ & $1.242(9)$ & $\begin{array}{c}2.4459(14) \\
2.512(2)\end{array}$ & - & 2231 \\
\hline$\left[\left(\eta^{5}-\mathrm{C}_{5} \mathrm{Me}_{4} \mathrm{H}\right)_{2} \operatorname{Pr}(\operatorname{thf})\right]_{2}\left(\mu-\eta^{2}: \eta^{2}-\mathrm{N}_{2}\right)^{\mathrm{c}}(\mathbf{1 8})[68]$ & $1.235(7)^{\mathrm{e}}$ & $\begin{array}{l}2.418(4)^{\mathrm{e}} \\
2.455(3)^{\mathrm{e}}\end{array}$ & - & 2383 \\
\hline$\left[\left(\eta^{5}-\mathrm{C}_{5} \mathrm{Me}_{4} \mathrm{H}\right)_{2} \mathrm{Nd}(\text { thf })\right]_{2}\left(\mu-\eta^{2}: \eta^{2}-\mathrm{N}_{2}\right)^{\mathrm{c}}(\mathbf{1 9})[79]$ & $1.241(5)^{\mathrm{e}}$ & $\begin{array}{l}2.404(3)^{\mathrm{e}} \\
2.451(2)^{\mathrm{e}}\end{array}$ & - & - \\
\hline$\left[\left(\eta^{5}-\mathrm{SiMe}_{3}-\mathrm{C}_{5} \mathrm{H}_{4}\right)_{2} \mathrm{Tm}(\mathrm{thf})\right]_{2}\left(\mu-\eta^{2}: \eta^{2}-\mathrm{N}_{2}\right)^{\mathrm{c}}(\mathbf{2 0})[75]$ & $1.236(8)$ & $\begin{array}{l}2.274(4) \\
2.302(4) \\
\end{array}$ & - & - \\
\hline$\left[\left(\eta^{5}-\mathrm{C}_{5} \mathrm{Me}_{4} \mathrm{H}\right)_{2} \mathrm{Lu}(\mathrm{thf})\right]_{2}\left(\mu-\eta^{2}: \eta^{2}-\mathrm{N}_{2}\right)^{\mathrm{c}}(\mathbf{2 1})[80]$ & $1.243(12)$ & $\begin{array}{l}2.290(6) \\
2.311(6) \\
\end{array}$ & - & 521 \\
\hline$\left[\left\{\left(\mathrm{Me}_{3} \mathrm{Si}\right)_{2} \mathrm{~N}\right\}_{2} \mathrm{Y}(\text { thf })\right]_{2}\left(\mu-\eta^{2}: \eta^{2}-\mathrm{N}_{2}\right)^{\mathrm{c}}(\mathbf{2 2})[81]$ & $1.274(3)$ & $\begin{array}{l}2.297(2) \\
2.308(2) \\
\end{array}$ & $\begin{array}{l}1425\left({ }^{14} \mathrm{~N}_{2}\right) \\
1377\left({ }^{15} \mathrm{~N}_{2}\right)\end{array}$ & $+513(\mathrm{t})$ \\
\hline$\left[\left\{\left(\mathrm{Me}_{3} \mathrm{Si}\right)_{2} \mathrm{~N}\right\}_{2} \mathrm{La}(\mathrm{thf})\right]_{2}\left(\mu-\eta^{2}: \eta^{2}-\mathrm{N}_{2}\right)(\mathbf{2 3})[81]$ & - & - & - & 516 \\
\hline$\left[\left\{\left(\mathrm{Me} e_{3} \mathrm{Si}\right)_{2} \mathrm{~N}\right\}_{2} \mathrm{Nd}(\text { thf })\right]_{2}\left(\mu-\eta^{2}: \eta^{2}-\mathrm{N}_{2}\right)^{\mathrm{c}}(\mathbf{2 4})[81]$ & $1.258(3)$ & $\begin{array}{l}2.3758(16) \\
2.3938(16) \\
\end{array}$ & - & - \\
\hline$\left[\left\{\left(\mathrm{Me} e_{3} \mathrm{Si}\right)_{2} \mathrm{~N}\right\}_{2} \mathrm{Gd}(\mathrm{thf})\right]_{2}\left(\mu-\eta^{2}: \eta^{2}-\mathrm{N}_{2}\right)^{\mathrm{c}}(\mathbf{2 5})[81]$ & $1.278(4)$ & $\begin{array}{l}2.326(2) \\
2.353(2)\end{array}$ & - & - \\
\hline$\left[\left\{\left(\mathrm{Me} \mathrm{S}_{3} \mathrm{Si}\right)_{2} \mathrm{~N}\right\}_{2} \mathrm{~Tb}(\mathrm{thf})\right]_{2}\left(\mu-\eta^{2}: \eta^{2}-\mathrm{N}_{2}\right)^{\mathrm{c}}(\mathbf{2 6})[81]$ & $1.271(4)$ & $\begin{array}{l}2.301(2) \\
2.328(2)\end{array}$ & - & - \\
\hline$\left[\left\{\left(\mathrm{Me}{ }_{3} \mathrm{Si}\right)_{2} \mathrm{~N}\right\}_{2} \mathrm{Dy}(\text { thf })\right]_{2}\left(\mu-\eta^{2}: \eta^{2}-\mathrm{N}_{2}\right)^{\mathrm{c}}(\mathbf{2 7})[82]$ & $1.305(6)$ & $\begin{array}{l}2.287(3) \\
2.312(3)\end{array}$ & - & - \\
\hline
\end{tabular}


Table 1. Cont.

\begin{tabular}{|c|c|c|c|c|}
\hline Complex (\#) [Reference] & $\begin{array}{l}\text { N-N Bond } \\
\text { Length }(\AA)\end{array}$ & $\begin{array}{c}\left.\text { Ln-N(N } \mathbf{N}_{2}\right) \\
\text { Bond } \\
\text { Lengths }(\AA ̊) \\
\end{array}$ & $\begin{array}{c}\mathrm{N}-\mathrm{N} \\
\text { Frequency } \\
\left(\mathrm{cm}^{-1}\right)\end{array}$ & $\begin{array}{c}{ }^{14 / 15} \mathrm{~N}-\mathrm{NMR} \\
\text { Spectroscopy } \\
(\mathrm{ppm})^{\mathrm{a}} \\
\end{array}$ \\
\hline$\left[\left\{\left(\mathrm{Me}_{3} \mathrm{Si}\right)_{2} \mathrm{~N}\right\}_{2} \mathrm{Ho}(\text { thf })\right]_{2}\left(\mu-\eta^{2}: \eta^{2}-\mathrm{N}_{2}\right)^{\mathrm{c}}(\mathbf{2 8})[83]$ & $1.264(4)$ & $\begin{array}{l}2.296(2) \\
2.315(2)\end{array}$ & - & - \\
\hline$\left[\left\{(\mathrm{Me})_{3} \mathrm{Si}\right)_{2} \mathrm{~N}\right\}_{2} \operatorname{Er}($ thf $\left.)\right]_{2}\left(\mu-\eta^{2}: \eta^{2}-\mathrm{N}_{2}\right)^{\mathrm{c}}(\mathbf{2 9})[81]$ & $1.276(5)$ & $\begin{array}{l}2.271(3) \\
2.302(3)\end{array}$ & - & - \\
\hline$\left[\left\{\left(\mathrm{Me}{ }_{3} \mathrm{Si}\right)_{2} \mathrm{~N}\right\}_{2} \mathrm{Tm}(\text { thf })\right]_{2}\left(\mu-\eta^{2}: \eta^{2}-\mathrm{N}_{2}\right)^{\mathrm{c}}(\mathbf{3 0})[82]$ & $1.261(4)$ & $\begin{array}{l}2.271(2) \\
2.296(2)\end{array}$ & - & - \\
\hline$\left[\left\{\left(\mathrm{Me}{ }_{3} \mathrm{Si}\right)_{2} \mathrm{~N}\right\}_{2} \mathrm{Lu}(\text { thf })\right]_{2}\left(\mu-\eta^{2}: \eta^{2}-\mathrm{N}_{2}\right)^{\mathrm{c}}(\mathbf{3 1})[83]$ & $1.285(4)$ & $\begin{array}{l}2.241(2) \\
2.272(2)\end{array}$ & $1451\left({ }^{14} \mathrm{~N}_{2}\right)$ & 557 \\
\hline$\left[\left\{\left(\mathrm{Me} e_{3} \mathrm{Si}\right)_{2} \mathrm{~N}\right\}_{2} \mathrm{Y}(\mathrm{PhCN})\right]_{2}\left(\mu-\eta^{2}: \eta^{2}-\mathrm{N}_{2}\right)^{\mathrm{c}}(\mathbf{3 2})[84]$ & $1.258(2)$ & $\begin{array}{l}2.2848(13) \\
2.3092(13)\end{array}$ & - & - \\
\hline$\left[\left\{\left(\mathrm{Me}{ }_{3} \mathrm{Si}\right)_{2} \mathrm{~N}\right\}_{2} \mathrm{Y}\left(\mathrm{C}_{5} \mathrm{H}_{5} \mathrm{~N}\right)\right]_{2}\left(\mu-\eta^{2}: \eta^{2}-\mathrm{N}_{2}\right)^{\mathrm{c}}(\mathbf{3 3})[84]$ & $1.255(3)$ & $\begin{array}{l}2.2917(16) \\
2.3107(17)\end{array}$ & - & - \\
\hline$\left[\left\{\left(\mathrm{Me}_{3} \mathrm{Si}\right)_{2} \mathrm{~N}\right\}_{2} \mathrm{Y}\left(4-\mathrm{NMe}_{2}-\mathrm{C}_{5} \mathrm{H}_{4} \mathrm{~N}\right)\right]_{2}\left(\mu-\eta^{2}: \eta^{2}-\mathrm{N}_{2}\right)^{\mathrm{c}}(\mathbf{3 4})[84]$ & $1.259(2)$ & $\begin{array}{l}2.2979(12) \\
2.3132(12)\end{array}$ & - & - \\
\hline$\left[\left\{\left(\mathrm{Me}{ }_{3} \mathrm{Si}\right)_{2} \mathrm{~N}\right\}_{2} \mathrm{Y}(\mathrm{Ph} 33 \mathrm{PO})\right]_{2}\left(\mu-\eta^{2}: \eta^{2}-\mathrm{N}_{2}\right){ }^{\mathrm{c}}(\mathbf{3 5})[84]$ & $1.262(2)$ & $\begin{array}{l}2.3000(14) \\
2.3022(14)\end{array}$ & - & - \\
\hline$\left[\left\{\left(\mathrm{Me} e_{3} \mathrm{Si}\right)_{2} \mathrm{~N}\right\}_{2} \mathrm{Y}\left(\mathrm{Me}_{3} \mathrm{NO}\right)\right]_{2}\left(\mu-\eta^{2}: \eta^{2}-\mathrm{N}_{2}\right)^{\mathrm{c}}(\mathbf{3 6})[84]$ & $1.198(3)$ & $\begin{array}{l}2.2925(17) \\
2.2941(18)\end{array}$ & - & - \\
\hline$\left[\left(2,6-{ }^{t} \mathrm{Bu}-\mathrm{C}_{6} \mathrm{H}_{3} \mathrm{O}\right)_{2} \mathrm{Nd}(\mathrm{thf})_{2}\right]_{2}\left(\mu-\eta^{2}: \eta^{2}-\mathrm{N}_{2}\right)(37)[82]$ & $1.242(7)$ & $\begin{array}{l}2.397(4) \\
2.401(3)\end{array}$ & - & - \\
\hline$\left[\left(2,6-{ }^{t} \mathrm{Bu}-\mathrm{C}_{6} \mathrm{H}_{3} \mathrm{O}\right)_{2} \mathrm{Dy}(\text { thf })_{2}\right]_{2}\left(\mu-\eta^{2}: \eta^{2}-\mathrm{N}_{2}\right)(\mathbf{3 8})[82]$ & $1.257(7)^{\mathrm{f}}$ & $\begin{array}{l}2.328(4)^{f} \\
2.340(4)^{f} \\
2.336(5)^{g} \\
2.336(5)^{g} \\
\end{array}$ & $1526\left({ }^{14} \mathrm{~N}_{2}\right)$ & - \\
\hline $\begin{array}{c}{\left[\mathrm{Na}_{4}(\mathrm{thf})_{8}\right]\left[\left(\eta^{5}: \eta^{1}: \eta^{5}: \eta^{1}-{ }^{\mathrm{Et} 2} \text { calix }[4] \text { pyrrole }\right) \operatorname{Pr}\right]_{2}\left(\mu-\eta^{2}: \eta^{2}-\mathrm{N}_{2}\right)(\mathbf{3 9})} \\
{[85]}\end{array}$ & - & - & - & - \\
\hline $\begin{array}{c}{\left[\mathrm{Na}_{4}(\mathrm{dme})_{5}\right]\left[\left(\eta^{5}: \eta^{1}: \eta^{5}: \eta^{1}-\mathrm{Et} 2 \text { calix }[4] \text { pyrrole }\right) \operatorname{Pr}\right]_{2}\left(\mu-\eta^{2}: \eta^{2}-\mathrm{N}_{2}\right)} \\
\text { (40) }[85]\end{array}$ & $1.254(7)$ & $\begin{array}{l}2.414(5) \\
2.457(5)\end{array}$ & - & - \\
\hline $\begin{array}{c}{\left[\mathrm{Na}_{4}(\mathrm{thf}) 8\right]\left[\left(\eta^{5}: \eta^{1}: \eta^{5}: \eta^{1}-\mathrm{Et}{ }^{2} \text { calix }[4] \text { pyrrole }\right) \mathrm{Nd}\right]_{2}\left(\mu-\eta^{2}: \eta^{2}-\mathrm{N}_{2}\right)(\mathbf{4 1})} \\
{[85]}\end{array}$ & - & - & - & - \\
\hline $\begin{array}{c}{[\mathrm{Na} 4(\text { dioxane }) 6]\left[\left(\eta^{5}: \eta^{1}: \eta^{5}: \eta^{1}-{ }^{\mathrm{Et} 2} \text { calix }[4] \text { pyrrole }\right) \mathrm{Nd}_{2}\left(\mu-\eta^{2}: \eta^{2}-\mathrm{N}_{2}\right)\right.} \\
\text { (42) }[85]\end{array}$ & $1.234(8)$ & $\begin{array}{l}2.511(4) \\
2.508(4)\end{array}$ & - & - \\
\hline $\begin{array}{c}{\left[\left\{\mathrm{HB}\left(3-{ }^{t} \mathrm{Bu}-5-\mathrm{Me}-\mathrm{pz}\right)\right\} \operatorname{Tm}\left\{\mathrm{NH}\left(2,5-{ }^{t} \mathrm{Bu}-\mathrm{C}_{6} \mathrm{H}_{3}\right)\right\}\right] 2\left(\mu-\eta^{2}: \eta^{2}-\mathrm{N}_{2}\right)} \\
(\mathbf{4 3})[86]\end{array}$ & $1.215(10)$ & $\begin{array}{l}2.274(8) \\
2.286(9)\end{array}$ & - & - \\
\hline
\end{tabular}

${ }^{\text {a }}$ Referenced to $\mathrm{CH}_{3}{ }^{15} \mathrm{NO} ;{ }^{\text {b }}$ In equilibrium with $\left[\left(\eta^{5}-\mathrm{C}_{5} \mathrm{Me}_{5}\right)_{2} \mathrm{Sm}\right] 2$; ${ }^{\mathrm{c}}$ trans arrangement of donor solvent; ${ }^{\mathrm{d}}$ cis arrangement of donor solvent; ${ }^{\mathrm{e}}$ The authors indicate poor data quality or significant disorder in these structures; ${ }^{\mathrm{f}}$ thf solvent of crystallisation in unit cell; ${ }^{\mathrm{g}}$ toluene solvent of crystallisation in unit cell.

\subsubsection{Cyclopentadienyl Ancillary Ligands}

The first isolated, structurally characterised dinitrogen complex of an f-element metal was reported by Evans and co-workers [72]. $\left[\left(\eta^{5}-\mathrm{C}_{5} \mathrm{Me}_{5}\right)_{2} \mathrm{Sm}\right]_{2}\left(\mu-\eta^{2}: \eta^{2}-\mathrm{N}_{2}\right)(4)$ was isolated by slow crystallisation 
of a toluene solution of the bent metallocene $\left[\left(\eta^{5}-\mathrm{C}_{5} \mathrm{Me}_{5}\right)_{2} \mathrm{Sm}\right]_{2}$ under an $\mathrm{N}_{2}$ atmosphere (Figure 2). 4 exists in dynamic equilibrium with the metallocene starting material involving reversible $\mathrm{Sm}^{\mathrm{II}} / \mathrm{Sm}^{\mathrm{III}}$ interconversion. In the solid state, 4 displays tetrahedral coordination around each $\mathrm{Sm}$ centre with gearing of the $\left[\mathrm{Sm}\left(\mathrm{C}_{5} \mathrm{Me}_{5}\right)_{2}\right]$ units and the first example of a co-planar $\mathrm{M}_{2} \mathrm{~N}_{2}$ diamond core for any metal. The bridging, side-on bound $\mathrm{N}_{2}$ has a short $\mathrm{N}-\mathrm{N}$ distance of 1.088(12) $\AA$ (free $\mathrm{N}_{2}: 1.0975 \AA$ [66]) and does not imply reduction to $\mathrm{N}_{2}{ }^{2-}$; however, recent studies by Arnold and co-workers have shown that $\mathrm{N}-\mathrm{N}$ bond lengths determined using $\mathrm{X}$-ray diffraction experiments can be underestimated and so may not provide the best way of assessing the level of dinitrogen reduction [87,88]. Both the Sm-N/C bond lengths and the ${ }^{13} \mathrm{C}-\mathrm{NMR}$ spectral data support formulation of the complex as $\left[\mathrm{Sm}^{\mathrm{III}}\right]_{2}\left(\mathrm{~N}_{2}{ }^{2-}\right)$. Maron and co-workers have reported calculations on the interaction of $\mathrm{N}_{2}$ with $\left[\left(\eta^{5}-\mathrm{C}_{5} \mathrm{Me}_{5}\right)_{2} \mathrm{Ln}\right](\mathrm{Ln}=$ $\mathrm{Sm}, \mathrm{Eu}, \mathrm{Yb}$ ) [89].

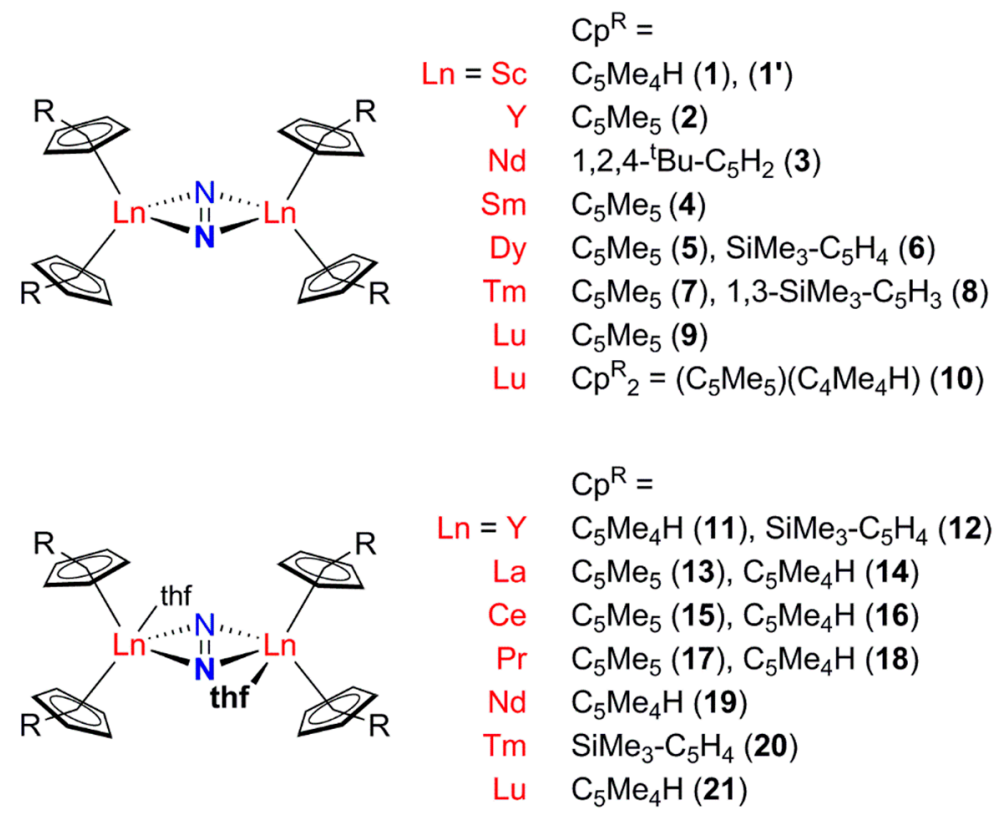

Figure 2. Rare earth complexes with cyclopentadienyl ligands resulting from $\mathrm{N}_{2}$ activation to $\mathrm{N}_{2}^{2-}$.

Since this landmark discovery, the methodology of using reducing divalent rare earth metal complexes to activate $\mathrm{N}_{2}$ has resulted in analogous cyclopentadienyl complexes of Dy $(\mathbf{5}, \mathbf{6})[74]$ and $\operatorname{Tm}(7,20)$ [75]. Structurally, these complexes all demonstrate a common planar $\mathrm{Ln}_{2} \mathrm{~N}_{2}$ core $(\mathrm{Ln}-\mathrm{N}-$ $\mathrm{N}$-Ln dihedral angle $=0^{\circ}$ ), with the arrangement of the cyclopentadienyl ligands being dependent on the metal centre and the nature of the ligand itself (Figure 3).

The number of dinitrogen complexes has been expanded significantly by the report that combination of trivalent lanthanide complexes $\mathrm{LnCp}^{\mathrm{R}}{ }_{3}$ or $\mathrm{LnCp}^{\mathrm{R}}{ }_{2} \mathrm{~A}^{\prime}$ with an alkali metal can also reduce dinitrogen affording side-on bound $\mathrm{N}_{2}$ complexes $\left[\left(\eta^{5}-\mathrm{Cp}^{\mathrm{R}}\right)_{2} \mathrm{Ln}\right]_{2}\left(\mu-\eta^{2}: \eta^{2}-\mathrm{N}_{2}\right)(\mathrm{Ln}=\operatorname{Sc}(\mathbf{1})$, $\mathrm{Y}(\mathbf{2}), \mathrm{Nd}(\mathbf{3})$, Dy $(\mathbf{5}, \mathbf{6}), \mathrm{Lu}(\mathbf{9}))[67,69-71,76]$ or $\left[\left(\eta^{5}-\mathrm{Cp}^{\mathrm{R}}\right)_{2} \operatorname{Ln}(\operatorname{thf})\right]_{2}\left(\mu-\eta^{2}: \eta^{2}-\mathrm{N}_{2}\right)(\operatorname{Ln}=\mathrm{Y}(\mathbf{1 1}, \mathbf{1 2})$, La $(\mathbf{1 3}, \mathbf{1 4}), \mathrm{Ce}(\mathbf{1 5}, \mathbf{1 6}), \operatorname{Pr}(\mathbf{1 7}, \mathbf{1 8}), \mathrm{Nd}(\mathbf{1 9}), \mathrm{Lu}(\mathbf{2 1}))[68,78-80,83]$. The generality of this method has been demonstrated by the wide range of metals utilised as well as the use of both homo- and heteroleptic trivalent lanthanide starting materials with a variety of cyclopentadienyl, amide, aryloxide, hydride, halide and borohydride ligands. 

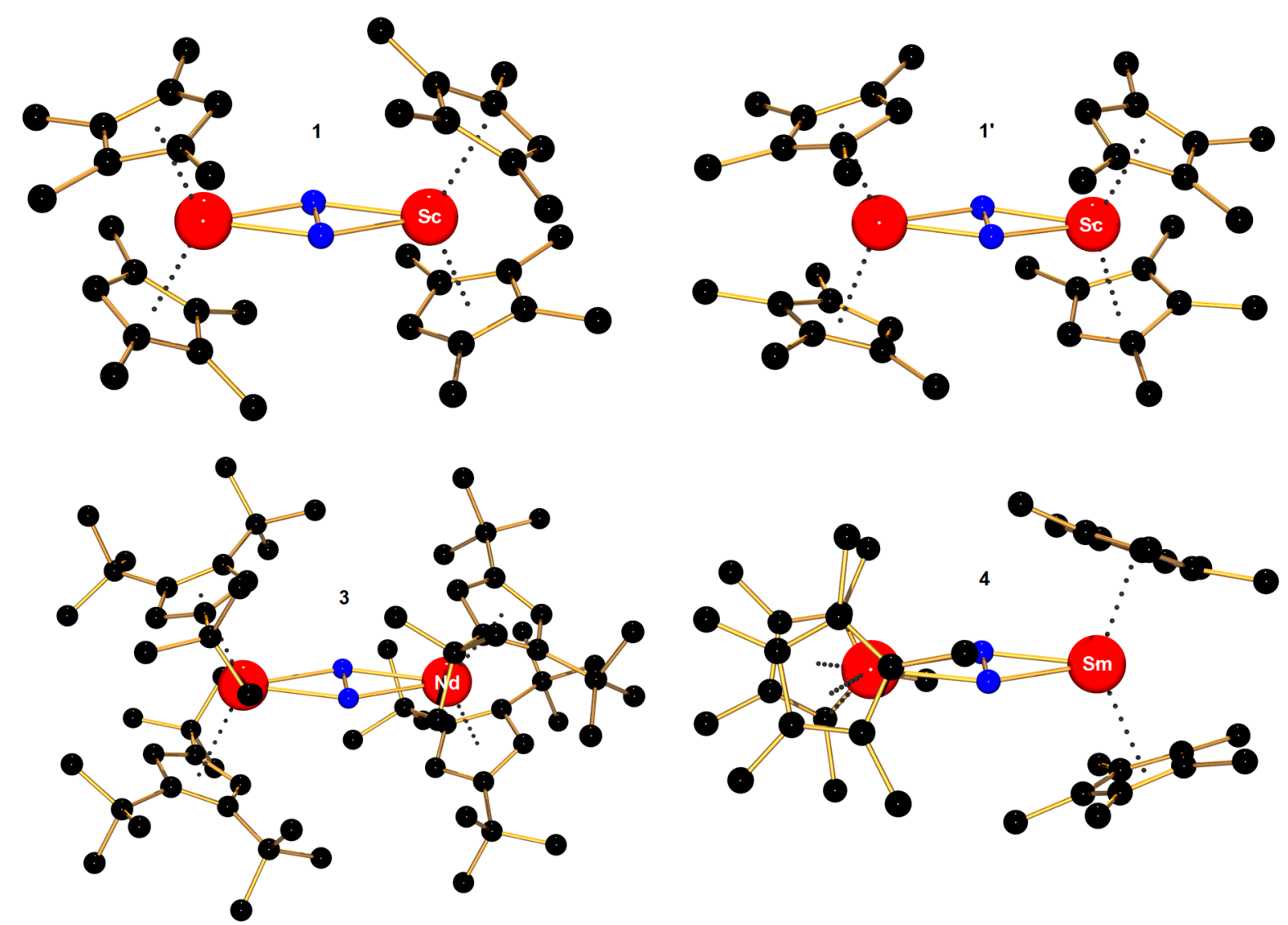

Figure 3. Structural variations in the solid state structures of $\left[\left(\eta^{5}-\mathrm{Cp}^{\mathrm{R}}\right)_{2} \mathrm{Ln}\right]_{2}\left(\mu-\eta^{2}: \eta^{2}-\mathrm{N}_{2}\right)$ $\left(\operatorname{Ln}=\mathrm{Sc}(\mathbf{1})\right.$ and $\left.\left(\mathbf{1}^{\prime}\right) ; \mathrm{Nd}(\mathbf{3}) ; \mathrm{Sm}(\mathbf{4})\right)$.

Complexes 11-21 (with donor solvent bound) also have planar $\mathrm{Ln}_{2} \mathrm{~N}_{2}$ cores. However, only 13 (La), $15(\mathrm{Ce})$ and $17(\mathrm{Pr})$ have a cis arrangement of thf molecules and an asymmetrically-bound $\mathrm{N}_{2}{ }^{2-}$ ligand (as a result of crystallographically non-equivalent $\mathrm{N}$ atoms). This is most clearly seen though the difference in $\mathrm{Ln}-\mathrm{N}-\mathrm{Ln}^{\prime}$ angles (11: 145.77(16) and 157.33(18) $; 1$ 13: 156.9(3) and 144.7(3) ; 15: 157.0(3) and $\left.145.1(3)^{\circ}\right)($ Figure 4$)$.

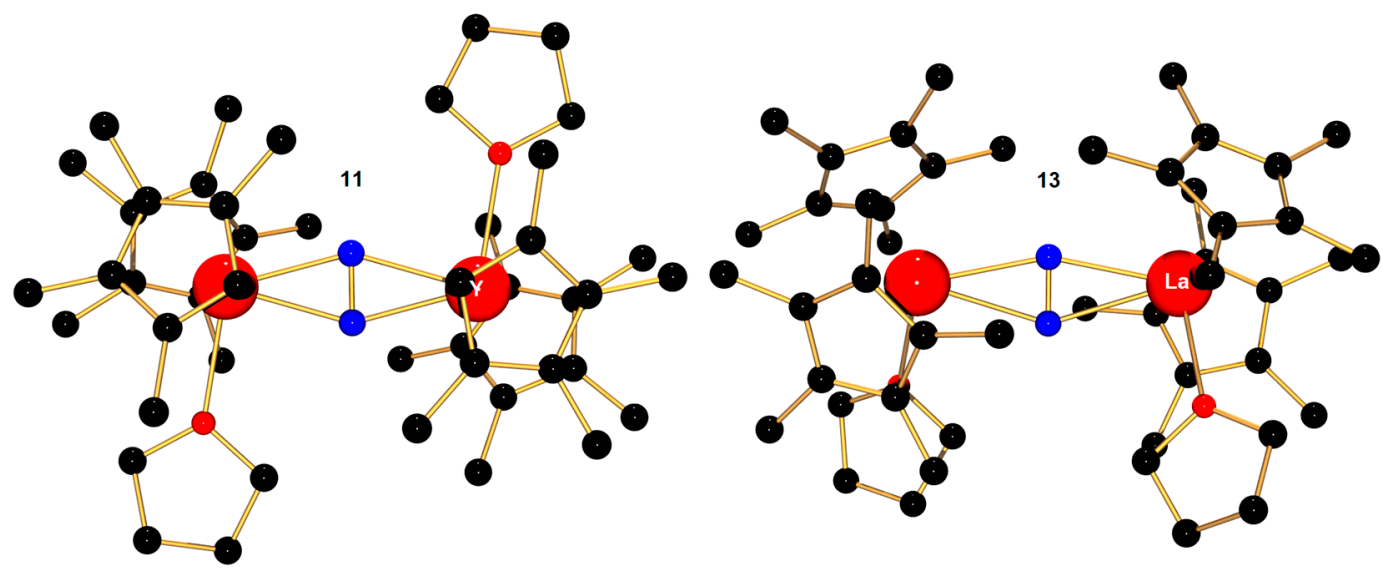

Figure 4. Structural variations in the solid state structures of $\left[\left(\eta^{5}-\mathrm{C}_{5} \mathrm{Me}_{4} \mathrm{H}\right)_{2} \mathrm{Ln}(\mathrm{thf})\right]_{2}$ $\left(\mu-\eta^{2}: \eta^{2}-\mathrm{N}_{2}\right)(\mathbf{1 1}, 14,16,18,19,21)(11$ depicted, left $)$ and $\left[\left(\eta^{5}-\mathrm{C}_{5} \mathrm{Me}\right)_{2} \operatorname{Ln}(\text { thf })\right]_{2}\left(\mu-\eta^{2}: \eta^{2}-\right.$ $\left.\mathrm{N}_{2}\right)(13,15,17)\left(13\right.$ depicted, right). One $\mathrm{C}_{5} \mathrm{Me}_{4}$ ring in 11 is disordered. 
In terms of bonding, calculations were carried out on 1 and the $\mathrm{Sc}-\mathrm{N}\left(\mathrm{N}_{2}\right)$ bonding interaction was found to be a polar covalent two-electron four-centre bond resulting from donation from a filled Sc $3 \mathrm{~d}$ orbital into an empty $\mathrm{N}_{2} \pi_{\mathrm{g}}$ antibonding orbital in the $\mathrm{Sc}_{2} \mathrm{~N}_{2}$ plane. The lowest unoccupied molecular orbital (LUMO) of $\mathbf{1}$ is an unperturbed antibonding $\pi_{\mathrm{g}}$ orbital based on $\mathrm{N}_{2}$. This bonding scheme can likely be extended for all compounds 1-21, with the donor nd orbital varying based on the nature of the metal ion [69].

Complexes $\mathbf{4}$ and 15-18 were characterised by ${ }^{15} \mathrm{~N}-\mathrm{NMR}$ spectroscopy; the first reported examples of such spectra for paramagnetic $\mathrm{N}_{2}$ complexes [68]. Trivalent $\mathrm{Sm}, \mathrm{Ce}$ and $\mathrm{Pr}$ were chosen due to the low magnetic susceptibility of these ions $\left(4 \mathrm{f}^{5} \mathrm{Sm}^{\mathrm{III}}, \mu=0.84 \mu_{\mathrm{B}} ; 4 \mathrm{f}^{1} \mathrm{Ce}^{\mathrm{III}}, \mu=2.54 \mu_{\mathrm{B}} ; 4 \mathrm{f}^{2} \operatorname{Pr}^{\mathrm{III}}\right.$, $\mu=3.58 \mu_{\mathrm{B}}$ ). Broad singlets at high frequency were observed for 15 (871 ppm), 16 (1001 ppm), 17 (2231 ppm) and $18(2383 \mathrm{ppm})$. Consistent with the reversible $\mathrm{N}_{2}$ coordination to 4 , only a singlet at -75 ppm corresponding to free $\mathrm{N}_{2}$ is observed at $298 \mathrm{~K}$ [68]. Cooling resulted in a new resonance at $-117 \mathrm{ppm}$ at $263 \mathrm{~K}$ which shifted linearly to $-161 \mathrm{ppm}$ at $203 \mathrm{~K}$ and accounts for bound $\mathrm{N}_{2}$. In the context of pioneering NMR spectroscopic characterisation of organometallic complexes, the solid state ${ }^{15} \mathrm{~N}$ - and ${ }^{139}$ La-NMR spectra of $\mathbf{1 4 -}{ }^{\mathbf{1 5}} \mathbf{N}_{\mathbf{2}}$ have also been reported [90].

Most recently, it has been demonstrated by Evans and co-workers that photochemical activation of the closed shell $\mathrm{LnA}_{2} \mathrm{~A}^{\prime}$ complexes $\operatorname{Ln}\left(\eta^{5}-\mathrm{C}_{5} \mathrm{Me}_{5}\right)_{2}\left(\eta^{3}-\mathrm{C}_{5} \mathrm{Me}_{4} \mathrm{H}\right)$ or $\operatorname{Ln}\left(\eta^{5}-\mathrm{C}_{5} \mathrm{Me}_{5}\right)\left(\eta^{5}-\mathrm{C}_{5} \mathrm{Me}_{4} \mathrm{H}\right)\left(\eta^{3}-\right.$ $\left.\mathrm{C}_{5} \mathrm{Me}_{4} \mathrm{H}\right)$, which feature a novel $\eta^{3}$ binding mode of a cyclopentadienyl ligand, yields side-on dinitrogen complexes $\left[\left(\eta^{5}-\mathrm{C}_{5} \mathrm{Me}_{5}\right)_{2} \mathrm{M}\right]_{2}\left(\mu-\eta^{2}: \eta^{2}-\mathrm{N}_{2}\right)\left(\mathrm{M}=\mathrm{Y}(\mathbf{2})\right.$, Dy (5), Lu (9)) and $\left[\left(\eta^{5}-\mathrm{C}_{5} \mathrm{Me}_{5}\right)\left(\eta^{5}-\right.\right.$ $\left.\left.\mathrm{C}_{5} \mathrm{Me}_{4} \mathrm{H}\right) \mathrm{Lu}\right]_{2}\left(\mu-\eta^{2}: \eta^{2}-\mathrm{N}_{2}\right)$ (10), with concomitant formation of $\left(\mathrm{C}_{5} \mathrm{Me}_{4} \mathrm{H}\right)_{2}$ [73]. These reactions typically take place in under $5 \mathrm{~h}$ but, in the absence of photochemical activation, normally require a number of weeks. Full conversion to $\mathbf{2}$ and $\mathbf{9}$ is achieved via this methodology; this is notable given that other synthetic methods afforded yields of $26 \%-51 \%$ and $49 \%-59 \%$ respectively. Sterically induced reduction, typified by bulky $\mathrm{M}\left(\eta^{5}-\mathrm{C}_{5} \mathrm{Me}_{5}\right)_{3}$ complexes, does not account for this process since the less sterically hindered $\left[\mathrm{C}_{5} \mathrm{Me}_{4} \mathrm{H}\right]^{-}$ligand acts as the reductant [91]. Calculations support a mechanism involving electron transfer from the $\left[\eta^{3}-\mathrm{C}_{5} \mathrm{Me}_{4} \mathrm{H}\right]^{-}$ligand into an empty $4 \mathrm{~d}_{z^{2}}$ orbital on the metal centre. This affords a $\left[\mathrm{C}_{5} \mathrm{Me}_{4} \mathrm{H}\right] \cdot$ radical which dimerises, and excited $\left[\mathrm{Cp}_{2} \mathrm{M}\right]^{*} \mathrm{nd}^{1}$ fragment which reduces $\mathrm{N}_{2}$. Similarly, the allyl complexes $\operatorname{Ln}\left(\mathrm{C}_{5} \mathrm{Me}_{5}\right)_{2}\left(\eta^{3}-\mathrm{C}_{3} \mathrm{RH}_{4}\right)(\mathrm{R}=\mathrm{H}$ or $\mathrm{Me})$ can be photochemically activated to yield $\mathbf{2}$ and $\mathbf{9}$; in this case, propene and isobutene are observed as by-products due to $\mathrm{H}$-atom abstraction from solvent rather than radical dimerisation [92].

\subsubsection{Amide Ancillary Ligands}

The simple silylamide ligand $\left[\mathrm{N}\left(\mathrm{SiMe}_{3}\right)_{2}\right]^{-}$has also proved suitable to prepare related complexes of the form $\left[\left\{\left(\mathrm{Me}_{3} \mathrm{Si}\right)_{2} \mathrm{~N}\right\}_{2} \operatorname{Ln}(\text { thf })\right]_{2}\left(\mu-\eta^{2}: \eta^{2}-\mathrm{N}_{2}\right)(\mathrm{Ln}=\mathrm{Y}$ (22), La (23), Nd (24), Gd (25), Tb (26), Dy (27), Ho (28), Er (29), Tm (30), Lu (31)) (Figure 5, left) [65,81-83,93-95]. All complexes can be prepared using the $\mathrm{LnA}_{3} / \mathrm{M}$ or $\mathrm{LnA}_{2} \mathrm{~A}^{\prime} / \mathrm{M}$ method and in addition, 24, 27 and 30 can be prepared directly from the divalent starting materials $\mathrm{NdI}_{2}, \mathrm{DyI}_{2}$ and $\mathrm{TmI}_{2}(\text { thf) })_{3}$ respectively (though 24 is notable in that it is only isolated in 4\% yield using this synthetic approach) [82].

Lewis base coordination of yttrium complex 22 was investigated through a series of substitution reactions to afford $\left[\left\{\left(\mathrm{Me}_{3} \mathrm{Si}\right)_{2} \mathrm{~N}\right\}_{2} \mathrm{Ln}(\mathrm{L})\right]_{2}\left(\mu-\eta^{2}: \eta^{2}-\mathrm{N}_{2}\right)\left(\mathrm{L}=\mathrm{PhCN}(32), \mathrm{C}_{5} \mathrm{H}_{5} \mathrm{~N}\right.$ (33), 4- $\mathrm{NMe}_{2}-\mathrm{C}_{5} \mathrm{H}_{4} \mathrm{~N}$ (34), $\mathrm{Ph}_{3} \mathrm{PO}$ (35), $\mathrm{Me}_{3} \mathrm{NO}(\mathbf{3 6})$ ) (Figure 5, right) [84]. Varying the donor ligand had little effect on the 
planar $\mathrm{Ln}_{2} \mathrm{~N}_{2}$ structural core of 22 and 32-35, though the $\mathrm{N}-\mathrm{N}$ distance in $\mathbf{3 6}$ was unexpectedly short at 1.198(3) A. Similarly for complexes 1-21, calculations on $\left[\left\{\left(\mathrm{Me}_{3} \mathrm{Si}\right)_{2} \mathrm{~N}\right\}_{2} \mathrm{Y}(\mathrm{L})\right]_{2}\left(\mu-\eta^{2}: \eta^{2}-\mathrm{N}_{2}\right)$ (22) indicate that the $\mathrm{Y}-\mathrm{N}\left(\mathrm{N}_{2}\right)$ bonding interaction involves donation from a filled $\mathrm{Y} 4 \mathrm{~d}$ orbital into an antibonding $\mathrm{N}_{2} \pi_{\mathrm{g}}$ orbital in the $\mathrm{Y}_{2} \mathrm{~N}_{2}$ plane. The LUMO is an unperturbed antibonding $\mathrm{N}_{2} \pi_{\mathrm{g}}$ orbital [84,93]. UV-Vis spectra of 22 and 31-35 all contained a low energy, low intensity absorption around $700 \mathrm{~nm}$ which corresponds to the formally electric-dipole forbidden HOMO-LUMO $\left(\mathrm{a}_{\mathrm{g}}-\mathrm{ag}_{\mathrm{g}}\right)$ transition and act as a fingerprint of the electronic structure of the $\mathrm{Y}_{2} \mathrm{~N}_{2}$ core.

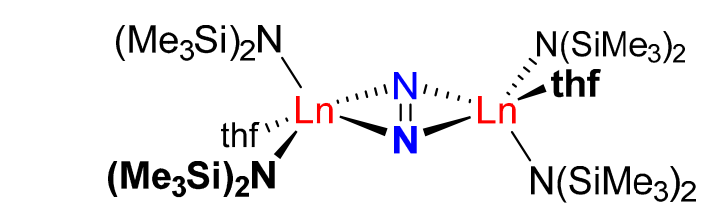

$\operatorname{Ln}=\mathrm{Y}(\mathbf{2 2}), \mathrm{La}(\mathbf{2 3}), \mathrm{Nd}(\mathbf{2 4}), \mathrm{Gd}(\mathbf{2 5}), \mathrm{Tb}(\mathbf{2 6})$

Dy (27), Ho (28), Er (29), Tm (30), Lu (31)

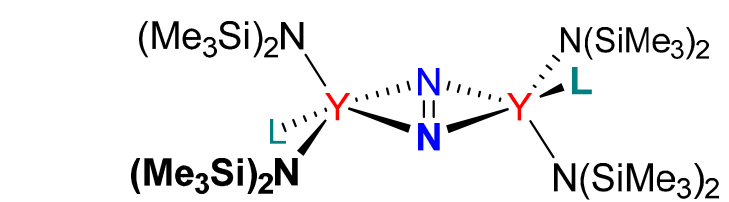

$\mathrm{L}=\mathrm{PhCN}$ (32), $\mathrm{C}_{5} \mathrm{H}_{5} \mathrm{~N}$ (33), 4- $\mathrm{NMe}_{2}-\mathrm{C}_{5} \mathrm{H}_{4} \mathrm{~N}$ (34)

$\mathrm{Ph}_{3} \mathrm{PO}$ (35), $\mathrm{Me}_{3} \mathrm{NO}$ (36)

Figure 5. Rare earth complexes with amide ligands resulting from $\mathrm{N}_{2}$ activation to $\mathrm{N}_{2}{ }^{2-}$.

In terms of bonding, the closed shell $4 \mathrm{f}^{14} \mathrm{Lu}^{\mathrm{III}}$ ion in $\mathbf{3 1}$ provides an interesting contrast to yttrium complex 22 with $4 \mathrm{f}^{\circ} \mathrm{Y}^{\mathrm{III}}$ ions. Calculations support that the bonding is described in analogy with 22, but using higher energy, radially diffuse $5 \mathrm{~d}$ orbitals which have a good energy match with the $\mathrm{N}_{2}$ antibonding $\pi_{\mathrm{g}}$ orbital and are the correct symmetry for overlap [65]. Hughbanks and co-workers have also reported calculations on the $4 \mathrm{f}^{7} \mathrm{Gd}^{\mathrm{III}}$ complex 33 to analyse magnetic coupling [96].

\subsubsection{Aryloxide Ancillary Ligands}

Aryloxide ancillary ligands have been used to prepare side-on $\mathrm{N}_{2}$ complexes of the form $\left[\left(2,6-{ }^{t} \mathrm{Bu}-\mathrm{C}_{6} \mathrm{H}_{3} \mathrm{O}\right)_{2} \mathrm{Ln}(\text { thf })_{2}\right]_{2}\left(\mu-\eta^{2}: \eta^{2}-\mathrm{N}_{2}\right) \quad(\mathrm{Ln}=\mathrm{Nd}(\mathbf{3 7})$ and Dy (38)) which now contain two molecules of coordinating solvent per metal (Figure 6) $[65,82,93]$. Calculations on 38, which contains open shell $4 \mathrm{f}^{9}$ Dy ${ }^{\mathrm{III}}$ ions, indicate that Dy- $\mathrm{N}_{2}$ bonding is derived from a $5 \mathrm{~d}-\pi_{\mathrm{g}}$ interaction in the $\mathrm{Dy} \mathrm{N}_{2}$ plane [65].

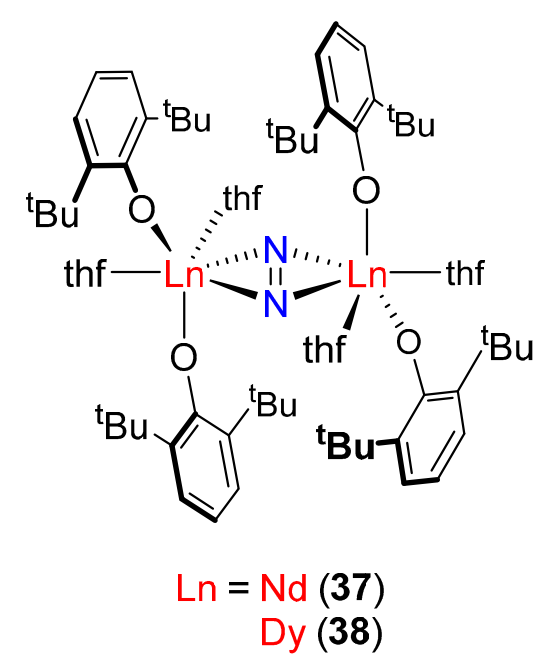

Figure 6. Rare earth complexes with aryloxide ligands resulting from $\mathrm{N}_{2}$ activation to $\mathrm{N}_{2}{ }^{2-}$. 


\subsubsection{Multidentate Ancillary Ligands}

Floriani and co-workers reported $\mathrm{N}_{2}$ complexes of $\operatorname{Pr}{ }^{\mathrm{III}}(\mathbf{3 9}, \mathbf{4 0})$ and $\mathrm{Nd}^{\mathrm{III}} \mathbf{( 4 1 , 4 2 )}$ using a calix[4]pyrrole ligand; these complexes were obtained as single crystals suitable for X-ray diffraction studies and no isolated yields were reported (Figure 7) [85]. [Na4(thf) 8$]\left[\left(\eta^{5}: \eta^{1}: \eta^{5}: \eta^{1}\right.\right.$ ${ }^{\mathrm{Et}}$ calix[4]pyrrole)Ln]2 $]_{2}\left(\mu-\eta^{2}: \eta^{2}-\mathrm{N}_{2}\right) \quad(\mathrm{Ln}=\operatorname{Pr}$ (39), $\mathrm{Nd}(41))$ were prepared by reduction of $\left[\mathrm{Na}(\mathrm{thf})_{2}\right]\left[\left(\eta^{5}: \eta^{1}: \eta^{5}: \eta^{1}{ }^{\mathrm{Et} 2}\right.\right.$ calix[4]pyrrole)Ln(thf)] ( $\mathrm{Ln}=\mathrm{Pr}$ or $\left.\mathrm{Nd}\right)$ using sodium metal with catalytic napthalene. Addition of dimethoxyethane (dme) to 39 led to solvent exchange to afford $\left[\mathrm{Na}_{4}(\mathrm{dme})_{5}\right]\left[\left(\eta^{5}: \eta^{1}: \eta^{5}: \eta^{1}{ }^{1}{ }^{\mathrm{Et} 2} \text { calix[4]pyrrole)Ln] }\right]_{2}\left(\mu-\eta^{2}: \eta^{2}-\mathrm{N}_{2}\right)(\mathbf{4 0})\right.$. Similarly, addition of dioxane to 41 affords [Na4(dioxane) 6$]\left[\left(\eta^{5}: \eta^{1}: \eta^{5}: \eta^{1}{ }^{\mathrm{E}}{ }^{2}\right.\right.$ calix[4] pyrrole)Ln] ${ }_{2}\left(\mu-\eta^{2}: \eta^{2}-\mathrm{N}_{2}\right)(\mathbf{4 2})$. The solid state structures of 40 and 42 revealed different coordination modes of the sodium counterions and feature $\mathrm{N}-\mathrm{N}$ bond distances of 1.254(7) and 1.234(8) $\AA$ which are consistent with reduction to $\mathrm{N}_{2}{ }^{2-}$. Measured magnetic moments are also consistent with $\mathrm{Ln}^{\mathrm{III}}$ oxidation state for both metal centres.

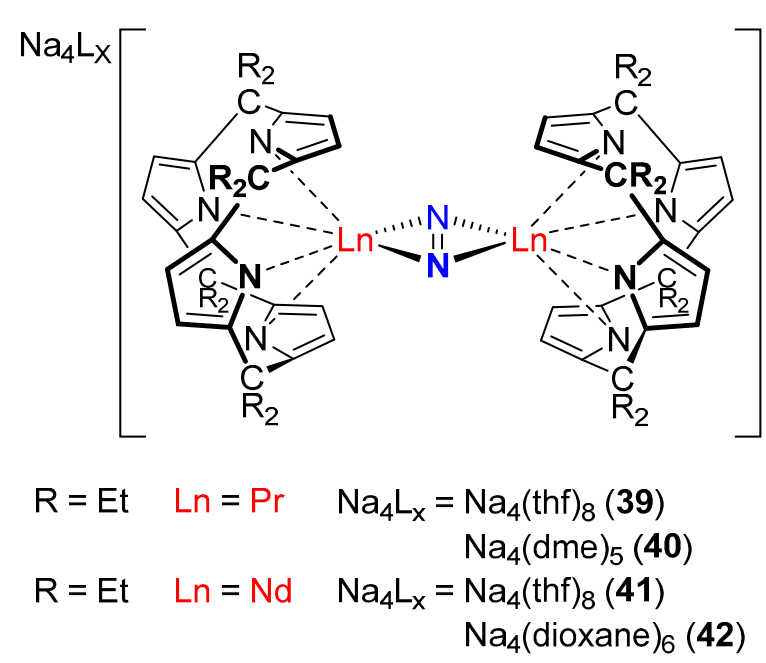

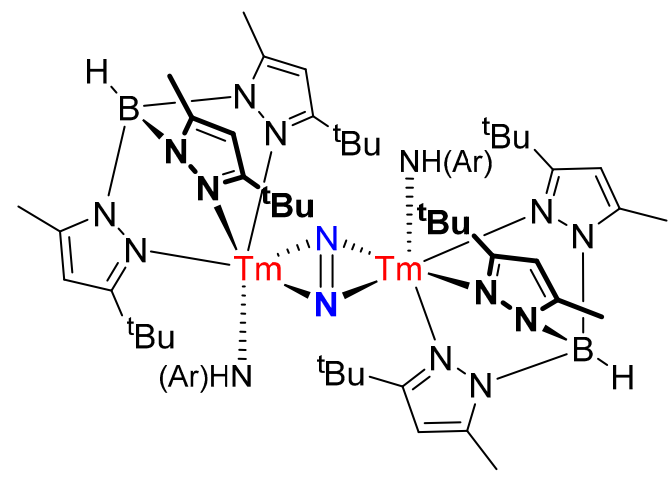

(43)

$\mathrm{Ar}=2,5-{ }^{\mathrm{t}} \mathrm{Bu}-\mathrm{C}_{6} \mathrm{H}_{3}$

Figure 7. Rare earth complexes with multidentate ligands resulting from $\mathrm{N}_{2}$ activation to $\mathrm{N}_{2}{ }^{2-}$.

Takats and co-workers reported the scorpionate complex $\left[\left\{\mathrm{HB}\left(3-{ }^{t} \mathrm{Bu}-5-\mathrm{Me}-\mathrm{pz}\right)\right\} \mathrm{Tm}\left\{\mathrm{NH}\left(2,5-{ }^{t} \mathrm{Bu}-\right.\right.\right.$ $\left.\left.\left.\mathrm{C}_{6} \mathrm{H}_{3}\right)\right\}\right]_{2}\left(\mu-\eta^{2}: \eta^{2}-\mathrm{N}_{2}\right)\left(\right.$ 43) $\left(\mathrm{pz}=\mathrm{C}_{3} \mathrm{HN}_{2}=\right.$ pyrazolyl) which was prepared by the protonolysis reaction of $2,5-{ }^{t} \mathrm{Bu}-\mathrm{C}_{6} \mathrm{H}_{3} \mathrm{NH}_{2}$ with the heteroleptic $\mathrm{Tm}^{\mathrm{II}}$ hydrocarbyl compound $\left\{\mathrm{HB}\left(3-{ }^{t} \mathrm{Bu}-5-\mathrm{Me}-\right.\right.$ $\mathrm{pz})\} \operatorname{Tm}\left\{\mathrm{CH}\left(\mathrm{SiMe}_{3}\right)_{2}\right\}$ [86]. The $\mathrm{N}-\mathrm{N}$ bond distance of 1.215(10) $\AA$ is consistent with reduction to $\mathrm{N}_{2}{ }^{2-}$ and the $\mathrm{Ln}_{2} \mathrm{~N}_{2}$ core is slightly bent with an $\mathrm{Ln}-\mathrm{N}-\mathrm{N}-\mathrm{Ln}$ torsion angle of $0.37^{\circ}$.

\subsection{Complexes Containing a Formal $\mathrm{N}_{2}{ }^{3-}$ Ligand}

\subsubsection{Amide Ancillary Ligands}

For reference, $\mathrm{N}-\mathrm{N}$ and $\mathrm{M}-\mathrm{N}\left(\mathrm{N}_{2}\right)$ bond lengths obtained from single crystal X-ray diffraction experiments and $\mathrm{N}-\mathrm{N}$ stretching frequencies (obtained by IR or Raman spectroscopy) are summarised in Table 2. 
Table 2. Summary of rare earth $\mathrm{N}_{2}{ }^{3-}$ complexes.

\begin{tabular}{|c|c|c|c|c|}
\hline Complex (\#) [Reference] & $\begin{array}{l}\text { N-N Bond } \\
\text { Length }(\AA)\end{array}$ & $\begin{array}{c}\text { Ln-N }\left(\mathbf{N}_{2}\right) \text { Bond } \\
\text { Lengths }(\AA)\end{array}$ & $\begin{array}{c}\text { Ln-N-N-Ln } \\
\text { Torsion } \\
\text { Angle }\left(^{\circ}\right) \\
\end{array}$ & $\begin{array}{c}\mathrm{N}-\mathrm{N} \\
\text { Frequency } \\
\left(\mathrm{cm}^{-1}\right) \\
\end{array}$ \\
\hline $\mathrm{N}_{2}$ & $1.0975[66]$ & - & - & $2331[67]$ \\
\hline \multirow{2}{*}[\mathrm{K}(\text{thf})_{6}]{$\left[\left\{\left(\mathrm{Me} \mathrm{S}_{3} \mathrm{Si}\right)_{2} \mathrm{~N}\right\}_{2} \mathrm{Y}(\text { thf })\right]_{2}\left(\mu-\eta^{2}: \eta^{2}-\mathrm{N}_{2}\right)^{\mathrm{b}}(\mathbf{4 4})[93]$} & $1.401(6)$ & $\begin{array}{l}2.194(3) \\
2.218(3)\end{array}$ & \multirow{2}{*}{0} & \multirow{2}{*}{$\begin{array}{l}1002\left({ }^{14} \mathrm{~N}_{2}\right) \\
\text { (calculated) }\end{array}$} \\
\hline & $1.401(6)^{\mathrm{a}}$ & $\begin{array}{l}2.190(3)^{\mathrm{a}} \\
2.213(3)^{\mathrm{a}}\end{array}$ & & \\
\hline$\left[\mathrm{K}(\text { thf })_{6}\right]\left[\left\{(\mathrm{Me} 3 \mathrm{Si})_{2} \mathrm{~N}\right\}_{2} \mathrm{La}(\text { thf })\right]_{2}\left(\mu-\eta^{2}: \eta^{2}-\mathrm{N}_{2}\right)(\mathbf{4 5})[65]$ & - & - & 0 & - \\
\hline$\left[\mathrm{K}(\text { thf })_{6}\right]\left[\left\{\left(\mathrm{Me}{ }_{3} \mathrm{Si}\right)_{2} \mathrm{~N}\right\}_{2} \mathrm{Lu}(\text { thf })\right]_{2}\left(\mu-\eta^{2}: \eta^{2}-\mathrm{N}_{2}\right)^{\mathrm{b}}(\mathbf{4 6})[65]$ & $1.414(8)$ & $\begin{array}{l}2.163(4) \\
2.180(4) \\
\end{array}$ & 0 & $979\left({ }^{14} \mathrm{~N}_{2}\right)$ \\
\hline$[\mathrm{K}]\left[\left\{\left(\mathrm{Me}{ }_{3} \mathrm{Si}\right)_{2} \mathrm{~N}\right\}_{2} \mathrm{Y}(\text { thf })\right]_{2}\left(\mu_{3}-\eta^{2}: \eta^{2}: \eta^{2}-\mathrm{N}_{2}\right)^{\mathrm{b}}(47)[93]$ & $1.405(3)$ & $\begin{array}{l}2.225(2) \\
2.242(2) \\
\end{array}$ & 14.22 & $\begin{array}{l}989\left({ }^{14} \mathrm{~N}_{2}\right) \\
956\left({ }^{15} \mathrm{~N}_{2}\right)\end{array}$ \\
\hline$[\mathrm{K}]\left[\left\{\left(\mathrm{Me}_{3} \mathrm{Si}\right){ }_{2} \mathrm{~N}\right\}_{2} \mathrm{Gd}(\mathrm{thf})\right]_{2}\left(\mu_{3}-\eta^{2}: \eta^{2}: \eta^{2}-\mathrm{N}_{2}\right){ }^{\mathrm{b}}(\mathbf{4 8})[97]$ & $1.395(3)$ & $\begin{array}{l}2.248(2) \\
2.274(2) \\
\end{array}$ & 13.64 & - \\
\hline$[\mathrm{K}]\left[\left\{\left(\mathrm{Me}_{3} \mathrm{Si}\right)_{2} \mathrm{~N}\right\}_{2} \mathrm{~Tb}(\text { thf })\right]_{2}\left(\mu_{3}-\eta^{2}: \eta^{2}: \eta^{2}-\mathrm{N}_{2}\right){ }^{\mathrm{b}}(\mathbf{4 9})[97]$ & $1.401(3)$ & $\begin{array}{l}2.235(2) \\
2.260(2)\end{array}$ & 16.12 & - \\
\hline$[\mathrm{K}]\left[\left\{\left(\mathrm{Me}_{3} \mathrm{Si}\right)_{2} \mathrm{~N}\right\}_{2} \mathrm{Dy}(\mathrm{thf})\right]_{2}\left(\mu_{3}-\eta^{2}: \eta^{2}: \eta^{2}-\mathrm{N}_{2}\right)^{\mathrm{b}}(\mathbf{5 0})[97]$ & $1.404(5)$ & $\begin{array}{l}2.229(4) \\
2.242(4)\end{array}$ & 15.27 & - \\
\hline $\begin{array}{c}{\left[\mathrm{K}(18 \mathrm{c} 6)(\text { thf })_{2}\right]\left[\left\{\left(\mathrm{Me}_{3} \mathrm{Si}\right)_{2} \mathrm{~N}\right\}_{2} \mathrm{Y}(\text { thf })\right]_{2}\left(\mu-\eta^{2}: \eta^{2}-\mathrm{N}_{2}\right)^{\mathrm{b}}} \\
\text { (51) [97] }\end{array}$ & $1.396(3)$ & $\begin{array}{l}2.1909(17) \\
2.2136(16)\end{array}$ & 0 & - \\
\hline $\begin{array}{c}{\left[\mathrm{K}(18 \mathrm{c} 6)(\text { thf })_{2}\right]\left[\left\{\left(\mathrm{Me} \mathrm{S}_{3} \mathrm{Si}\right)_{2} \mathrm{~N}\right\}_{2} \mathrm{Gd}(\mathrm{thf})\right]_{2}\left(\mu-\eta^{2}: \eta^{2}-\mathrm{N}_{2}\right)^{\mathrm{b}}(\mathbf{5 2})} \\
{[94]}\end{array}$ & $1.401(4)$ & $\begin{array}{l}2.224(2) \\
2.249(2)\end{array}$ & 0 & - \\
\hline $\begin{array}{c}{\left[\mathrm{K}(18 \mathrm{c} 6)(\text { thf })_{2}\right]\left[\left\{\left(\mathrm{Me} \mathrm{e}_{3} \mathrm{Si}\right)_{2} \mathrm{~N}\right\}_{2} \mathrm{~Tb}(\mathrm{thf})\right]_{2}\left(\mu-\eta^{2}: \eta^{2}-\mathrm{N}_{2}\right)^{\mathrm{b}}} \\
(\mathbf{5 3})[94]\end{array}$ & $1.394(3)$ & $\begin{array}{l}2.2056(15) \\
2.2345(15)\end{array}$ & 0 & - \\
\hline $\begin{array}{c}{\left[\mathrm{K}(18 \mathrm{c} 6)(\text { thf })_{2}\right]\left[\left\{\left(\mathrm{Me} \mathrm{e}_{3} \mathrm{Si}\right)_{2} \mathrm{~N}\right\}_{2} \mathrm{Dy}(\mathrm{thf})\right]_{2}\left(\mu-\eta^{2}: \eta^{2}-\mathrm{N}_{2}\right)^{\mathrm{b}}} \\
(\mathbf{5 4})[94]\end{array}$ & $1.393(7)$ & $\begin{array}{l}2.199(4) \\
2.213(4)\end{array}$ & 0 & - \\
\hline $\begin{array}{c}{\left[\mathrm{K}(18 \mathrm{c} 6)(\text { thf })_{2}\right]\left[\left\{\left(\mathrm{Me}{ }_{3} \mathrm{Si}\right)_{2} \mathrm{~N}\right\}_{2} \mathrm{Ho}(\mathrm{thf})\right]_{2}\left(\mu-\eta^{2}: \eta^{2}-\mathrm{N}_{2}\right){ }^{\mathrm{b}}} \\
(\mathbf{5 5})[95]\end{array}$ & $1.404(4)$ & $\begin{array}{l}2.188(2) \\
2.210(2)\end{array}$ & 0 & - \\
\hline $\begin{array}{c}{\left[\mathrm{K}(18 \mathrm{c} 6)(\text { thf })_{2}\right]\left[\left\{\left(\mathrm{Me}_{3} \mathrm{Si}\right)_{2} \mathrm{~N}\right\}_{2} \operatorname{Er}(\text { thf })\right]_{2}\left(\mu-\eta^{2}: \eta^{2}-\mathrm{N}_{2}\right)^{\mathrm{b}}} \\
(\mathbf{5 6})[65]\end{array}$ & $1.409(4)$ & $\begin{array}{l}2.178(2) \\
2.204(2)\end{array}$ & 0 & - \\
\hline$\left[\mathrm{Na}(\text { thf })_{6}\right]\left[\left\{\left(\mathrm{Me}_{3} \mathrm{Si}\right)_{2} \mathrm{~N}\right\}_{2} \mathrm{Y}(\text { thf })\right]_{2}\left(\mu-\eta^{2}: \eta^{2}-\mathrm{N}_{2}\right)^{\mathrm{b}}(\mathbf{5 7})[65]$ & $1.393(7)$ & $\begin{array}{l}2.199(4) \\
2.213(4) \\
\end{array}$ & 0 & - \\
\hline$\left[\mathrm{Na}(\text { thf })_{6}\right]\left[\left\{\left(\mathrm{Me} e_{3} \mathrm{Si}\right)_{2} \mathrm{~N}\right\}_{2} \operatorname{Er}(\text { thf })\right]_{2}\left(\mu-\eta^{2}: \eta^{2}-\mathrm{N}_{2}\right)^{\mathrm{b}}(\mathbf{5 8})[65]$ & $1.403(4)$ & $\begin{array}{l}2.1817(19) \\
2.2019(19)\end{array}$ & 0 & - \\
\hline $\begin{array}{c}{\left[\mathrm{K}(\text { thf })_{6}\right]\left[\left(2,6-{ }^{t} \mathrm{Bu}-\mathrm{C}_{6} \mathrm{H}_{3} \mathrm{O}\right)_{2} \mathrm{Dy}(\text { thf })\right]_{2}\left(\mu-\eta^{2}: \eta^{2}-\mathrm{N}_{2}\right)} \\
\text { (59) }[93]\end{array}$ & $1.396(7)$ & $\begin{array}{l}2.197(3) \\
2.203(4)\end{array}$ & 0 & $962\left({ }^{14} \mathrm{~N}_{2}\right)$ \\
\hline $\begin{array}{c}{[\mathrm{K}(\mathrm{thf})]\left[\left(2,6-{ }^{t} \mathrm{Bu}-\mathrm{C}_{6} \mathrm{H}_{3} \mathrm{O}\right)_{2} \mathrm{Dy}(\mathrm{thf})\right]_{2}\left(\mu_{3}-\eta^{2}: \eta^{2}: \eta^{2}-\mathrm{N}_{2}\right)(\mathbf{6 0})} \\
{[93]}\end{array}$ & $1.402(7)$ & $\begin{array}{l}2.235(5) \\
2.209(5)\end{array}$ & 6.59 & - \\
\hline
\end{tabular}

${ }^{a}$ Second independent molecule in unit cell; ${ }^{\mathrm{b}}$ trans arrangement of thf.

The first definitive evidence for an $\mathrm{N}_{2}{ }^{3-}$ reduction product of dinitrogen was demonstrated by Evans and co-workers [93]. The $\mathrm{LnA}_{3} / \mathrm{M}$ system of $\mathrm{Y}\left\{\mathrm{N}\left(\mathrm{SiMe}_{3}\right)_{2}\right\}_{3}$ with $\mathrm{KC}_{8}$ in thf afforded a mixture of $\left[\left\{\left(\mathrm{Me}{ }_{3} \mathrm{Si}\right)_{2} \mathrm{~N}\right\}_{2} \mathrm{Y}(\text { thf })\right]_{2}\left(\mu-\eta^{2}: \eta^{2}-\mathrm{N}_{2}\right)(\mathbf{2 2}),\left[\mathrm{K}(\text { thf })_{6}\right]\left[\left\{\left(\mathrm{Me} \mathrm{Si}_{3}\right)_{2} \mathrm{~N}\right\}_{2} \mathrm{Y}(\text { thf })\right]_{2}\left(\mu-\eta^{2}: \eta^{2}-\mathrm{N}_{2}\right)$ (44) and $[\mathrm{K}]\left[\left\{\left(\mathrm{Me}{ }_{3} \mathrm{Si}\right)_{2} \mathrm{~N}\right\}_{2} \mathrm{Y}(\text { thf })\right]_{2}\left(\mu_{3}-\eta^{2}: \eta^{2}: \eta^{2}-\mathrm{N}_{2}\right)(47)$ from which 44 and 47 could be isolated (Figure 8). 
The EPR spectrum of $\mathbf{4 4}^{\mathbf{1 5}} \mathbf{N}_{\mathbf{2}}$ has a 9-line pattern consistent with a triplet of triplets due to two ${ }^{15} \mathrm{~N}$ and two ${ }^{89} \mathrm{Y}$ nuclei and has a hyperfine coupling constant of $8.2 \mathrm{G}$ implying a N-centred radical, while ${ }_{47-15} \mathbf{N}_{2}$ shows extra coupling to potassium; all spectra indicate the presence of the $\mathrm{N}_{2}{ }^{3-}$ ion. The N-N bond distances are 1.401(6) and 1.405(3) $\AA$ respectively and are intermediate between $\mathrm{N}-\mathrm{N}$ single bonds $\left(1.47 \AA\right.$ in $\left.\mathrm{N}_{2} \mathrm{H}_{4}\right)$ and $\mathrm{N}=\mathrm{N}$ double bonds $(1.25 \AA$ in $\mathrm{PhN}=\mathrm{NPh})$ [98]. The $\mathrm{N}-\mathrm{N}$ vibrational stretching frequency in 47 is $989 \mathrm{~cm}^{-1}$, significantly reduced from $1425 \mathrm{~cm}^{-1}$ for 22 . Similarly to complexes 22-36, the $\mathrm{Y}-\mathrm{N}_{2}$ bonding interaction in these complexes can be described by the donation from a filled $\mathrm{Y} 4 \mathrm{~d}$ orbital into an antibonding $\mathrm{N}_{2} \pi_{\mathrm{g}}$ orbital (HOMO). However, the orthogonal antibonding $\mathrm{N}_{2} \pi_{\mathrm{g}}$ orbital is now also occupied by a single electron.

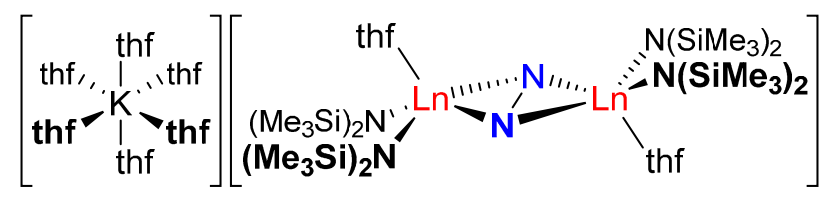

$\operatorname{Ln}=Y(\mathbf{4 4}), \operatorname{La}(\mathbf{4 5}), \operatorname{Lu}(\mathbf{4 6})$

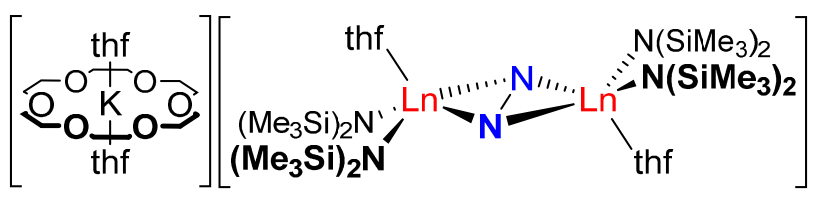

$\operatorname{Ln}=Y(51), \operatorname{Gd}(52), \operatorname{Tb}(53), \operatorname{Dy}(54)$, Ho (55), Er (56)

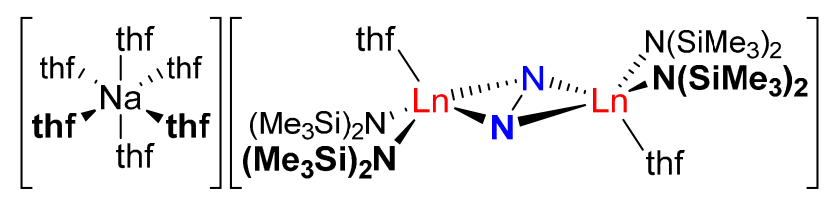

$$
\operatorname{Ln}=Y(57), \operatorname{Er}(58)
$$

Figure 8. Rare earth complexes with amide ligands resulting from $\mathrm{N}_{2}$ activation to $\mathrm{N}_{2}{ }^{3-}$.

Following this remarkable report, these types of complexes have been extended to more rare earth metal centres; $\left[\mathrm{K}(\text { thf })_{6}\right]\left[\left\{\left(\mathrm{Me}{ }_{3} \mathrm{Si}\right)_{2} \mathrm{~N}\right\}_{2} \operatorname{Ln}(\operatorname{thf})\right]_{2}\left(\mu-\eta^{2}: \eta^{2}-\mathrm{N}_{2}\right)(\mathrm{Ln}=\mathrm{La}(\mathbf{4 5}), \mathrm{Lu}(\mathbf{4 6}))$ and $[\mathrm{K}]\left[\left\{\left(\mathrm{Me}{ }_{3} \mathrm{Si}\right)_{2} \mathrm{~N}\right\}_{2} \operatorname{Ln}(\mathrm{thf})\right]_{2}\left(\mu_{3}-\eta^{2}: \eta^{2}: \eta^{2}-\mathrm{N}_{2}\right) \quad(\operatorname{Ln}=\mathrm{Gd}(\mathbf{4 8}), \mathrm{Tb}$ (49), Dy (50)). Additionally, the solvated counterion can also be varied to include 18-crown-6 in $\left[\mathrm{K}(18 \mathrm{c} 6)(\text { thf })_{2}\right]\left[\left\{\left(\mathrm{Me}_{3} \mathrm{Si}\right)_{2} \mathrm{~N}\right\}_{2} \mathrm{Ln}(\mathrm{thf})\right]_{2}$ $\left(\mu-\eta^{2}: \eta^{2}-\mathrm{N}_{2}\right)(\operatorname{Ln}=\mathrm{Y}(\mathbf{5 1}), \mathrm{Gd}(\mathbf{5 2}), \mathrm{Tb}(\mathbf{5 3})$, Dy (54), Ho (55), Er (56)) or can be exchanged for sodium in $\left[\mathrm{Na}(\text { thf })_{6}\right]\left[\left\{\left(\mathrm{Me}_{3} \mathrm{Si}\right)_{2} \mathrm{~N}\right\}_{2} \operatorname{Ln}(\text { thf })\right]_{2}\left(\mu-\eta^{2}: \eta^{2}-\mathrm{N}_{2}\right)(\operatorname{Ln}=\mathrm{Y}(\mathbf{5 7}), \operatorname{Er}(\mathbf{5 8}))[65,94,95,97]$.

The solid state structures of 44-46 and 51-58 which contain outer-sphere counterions have the common $\mathrm{Ln}_{2} \mathrm{~N}_{2}$ planar core like those of rare earth $\mathrm{N}_{2}{ }^{2-}$ complexes 1-42 which have been structurally characterised. However, complexes 47-50, which have inner-sphere $\mathrm{K}^{+}$ions, display bent $\mathrm{Ln}_{2} \mathrm{~N}_{2}$ cores with torsion angles of $14.22^{\circ}(\mathbf{4 7}), 13.64^{\circ}(\mathbf{4 8}), 16.12^{\circ}(\mathbf{4 9})$ and $15.27^{\circ}(\mathbf{5 0})$.

Complexes 48-50 (inner-sphere $\mathrm{K}^{+}$) and 52-56 (outer-sphere $\mathrm{K}^{+}$) all display interesting magnetic properties; 48 and $\mathbf{5 2}$ have the strongest magnetic exchange couplings in a $\mathrm{Gd}^{\mathrm{III}}$ complex with exchange constants of $-27 \mathrm{~cm}^{-1}$, and 49, 50 and 53-56 demonstrate single-molecule-magnet 
behaviour $[94,95,99,100]$. Combination of rare earth complexes which demonstrate both high anisotropy and strong exchange coupling potentially provides a route to single-molecule magnets with high blocking temperatures. The diffuse nature of the $\mathrm{N}_{2}{ }^{3-}$ radical facilitates strong coupling in these systems by overlap of the Ln $4 \mathrm{f}$ orbitals with the bridging dinitrogen ligand. 53 and 54 exhibit magnetic hysteresis up to record blocking temperatures of $13.9 \mathrm{~K}\left(0.9 \mathrm{mTs}^{-1}\right.$ sweep rate) and $8.3 \mathrm{~K}$ (0.08 $\mathrm{Ts}^{-1}$ sweep rate) respectively. Competing $\mathrm{Ln}^{\mathrm{III}}-\mathrm{Ln}^{\mathrm{III}}$ antiferromagnetic coupling is observed in complexes at low temperatures in $\mathbf{4 8 - 5 0}$, which have a non-zero $\mathrm{Ln}-\mathrm{N}-\mathrm{N}-\mathrm{Ln}$ dihedral angle, demonstrating the importance of geometry of the $\mathrm{Ln}_{2} \mathrm{~N}_{2}$ unit to magnetic behaviour.

\subsubsection{Aryloxide Ancillary Ligands}

As for yttrium complexes 44 and $\mathbf{4 7}$, the dysprosium aryloxide complexes $[\mathrm{K}(\mathrm{thf}) 6]\left[\left(2,6-{ }^{t} \mathrm{Bu}-\right.\right.$ $\left.\mathrm{C}_{6} \mathrm{H}_{3} \mathrm{O}\right)_{2} \mathrm{Dy}($ thf $\left.)\right]_{2}\left(\mu-\eta^{2}: \eta^{2}-\mathrm{N}_{2}\right)(\mathbf{5 9})$ and $[\mathrm{K}($ thf $)]\left[\left(2,6-{ }^{-}{ }^{\mathrm{Bu}}-\mathrm{C}_{6} \mathrm{H}_{3} \mathrm{O}\right)_{2} \mathrm{Dy}(\text { thf })\right]_{2}\left(\mu_{3}-\eta^{2}: \eta^{2}: \eta^{2}-\mathrm{N}_{2}\right)(\mathbf{6 0})$ were first isolated from reaction of $\mathrm{DyI}_{2}$ with $\mathrm{KO}-2,6-{ }^{t} \mathrm{Bu}-\mathrm{C}_{6} \mathrm{H}_{3}$ (Figure 9) $[65,93]$. Reoxidation of 59 with $\mathrm{AgBPh}_{4}$ affords the $\mathrm{N}_{2}{ }^{2-}$ complex $\left[\left(2,6-{ }^{t} \mathrm{Bu}-\mathrm{C}_{6} \mathrm{H}_{3} \mathrm{O}\right)_{2} \mathrm{Dy}(\mathrm{thf})_{2}\right]_{2}\left(\mu-\eta^{2}: \eta^{2}-\mathrm{N}_{2}\right)(\mathbf{3 8})$. From the solid state structures the $\mathrm{N}-\mathrm{N}$ bond lengths are 1.396(7) (59) and 1.402(7) $\AA$ (60), which fall in the range of reported $\mathrm{N}_{2}{ }^{3-}$ complexes (Table 2). As anticipated, 59 has a planar $\mathrm{Ln}_{2} \mathrm{~N}_{2}$ core in the solid state whereas it is bent in complex 60 with a $\mathrm{Ln}-\mathrm{N}-\mathrm{N}-\mathrm{Ln}$ torsion angle of $6.59^{\circ}$.

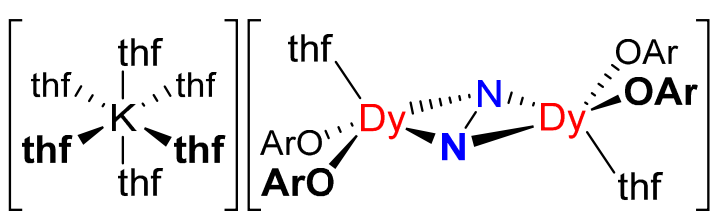

(59)

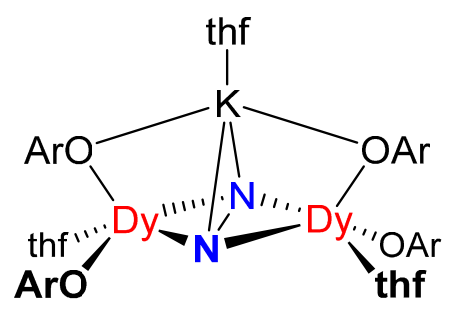

(60)

$$
\mathrm{Ar}=2,6-{ }^{\mathrm{t}} \mathrm{Bu}-\mathrm{C}_{6} \mathrm{H}_{3}
$$

Figure 9. Rare earth complexes with aryloxide ligands resulting from $\mathrm{N}_{2}$ activation to $\mathrm{N}_{2}{ }^{3-}$.

\subsection{Complexes Containing a Formally $\mathrm{N}_{2}{ }^{4-}$ Ligand}

For reference, $\mathrm{N}-\mathrm{N}$ and $\mathrm{M}-\mathrm{N}\left(\mathrm{N}_{2}\right)$ bond lengths obtained from single crystal X-ray diffraction experiments are summarised in Table 3. 
Table 3. Summary of rare earth $\mathrm{N}_{2}{ }^{4-}$ complexes.

\begin{tabular}{|c|c|c|c|}
\hline Complex (\#) [Reference] & $\begin{array}{l}\text { N-N Bond } \\
\text { Length }(\AA)\end{array}$ & $\begin{array}{c}\text { Ln-N }\left(\mathbf{N}_{2}\right) \\
\text { Bond Lengths } \\
(\text { Side-on) }(\AA) \\
\end{array}$ & $\begin{array}{c}\text { Ln-N }\left(\mathrm{N}_{2}\right) \\
\text { Bond Lengths } \\
(\text { End-on) }(\AA) \\
\end{array}$ \\
\hline $\mathrm{N}_{2}$ & $1.0975[66]$ & - & - \\
\hline$\left[\mathrm{Li}(\text { thf })_{2}\right]_{2}\left[\left({ }^{\mathrm{Et} 2}\right.\right.$ calix $[4]$ pyrrole $) \mathrm{Sm}_{2}\left(\mathrm{~N}_{2} \mathrm{Li}_{4}\right)(\mathbf{6 1})[101]$ & $1.525(4)$ & $\begin{array}{l}2.357(2) \\
2.342(2)\end{array}$ & - \\
\hline$\left[\left\{\mathrm{Ph}_{2} \mathrm{C}\left(\mathrm{C}_{4} \mathrm{H}_{3} \mathrm{~N}\right)_{2}\right\} \mathrm{Sm}(\mathrm{thf})\right]_{4}\left(\mu_{4}-\eta^{1}: \eta^{1}: \eta^{2}: \eta^{2}-\mathrm{N}_{2}\right)(\mathbf{6 2})[102]$ & $1.412(17)$ & $\begin{array}{l}2.327(3) \\
2.327(3)\end{array}$ & $\begin{array}{l}2.177(8) \\
2.177(8)\end{array}$ \\
\hline$\left[\left\{\mathrm{CyC}\left(\mathrm{C}_{4} \mathrm{H}_{3} \mathrm{~N}\right)_{2}\right\} \operatorname{Sm}(\text { thf })\right]_{4}\left(\mu_{4}-\eta^{1}: \eta^{1}: \eta^{2}: \eta^{2}-\mathrm{N}_{2}\right)(\mathbf{6 3})[103]$ & $1.392(16)$ & $\begin{array}{l}2.339(3) \\
2.339(3)\end{array}$ & $\begin{array}{l}2.160(8) \\
2.160(8)\end{array}$ \\
\hline$\left[\left\{\mathrm{Et}_{2} \mathrm{C}\left(\mathrm{C}_{4} \mathrm{H}_{3} \mathrm{~N}\right)_{2}\right\} \operatorname{Sm}(\text { thf })\right]_{4}\left(\mu_{4}-\eta^{1}: \eta^{1}: \eta^{2}: \eta^{2}-\mathrm{N}_{2}\right)(\mathbf{6 4})[104]$ & $1.415(4)$ & $\begin{array}{l}2.328(3) \\
2.342(3)\end{array}$ & $2.145(3)$ \\
\hline$\left[\left\{\mathrm{Ph}(\mathrm{Me}) \mathrm{C}\left(\mathrm{C}_{4} \mathrm{H}_{3} \mathrm{~N}\right)_{2}\right\} \mathrm{Sm}(\mathrm{dme})\right]_{4}\left(\mu_{4}-\eta^{1}: \eta^{1}: \eta^{2}: \eta^{2}-\mathrm{N}_{2}\right)(\mathbf{6 5})[104]$ & $1.42(2)$ & $\begin{array}{l}2.316(13) \\
2.316(12)\end{array}$ & $2.149(11)$ \\
\hline $\begin{array}{c}{[\mathrm{Na}(\text { thf })]_{2}\left[\left\{\mathrm{CyC}\left(\mathrm{C}_{4} \mathrm{H}_{3} \mathrm{~N}\right)_{2}\right\} \mathrm{Sm}(\mathrm{thf})\right]_{4}\left(\mu_{6}-\eta^{1}: \eta^{1}: \eta^{1}: \eta^{1}: \eta^{2}: \eta^{2}-\mathrm{N}_{2}\right)} \\
(\mathbf{6 6})[103]\end{array}$ & $1.371(16)$ & $\begin{array}{l}2.332(11) \\
2.324(11)\end{array}$ & $2.178(10)$ \\
\hline $\begin{array}{c}{\left[\{\mathrm{Li}(\mathrm{thf})\}_{3}\left(\mu_{3}-\mathrm{Cl}\right)\right]\left[\left({ }^{\mathrm{Cy}} \text { calix }[4] \text { pyrrole }\right) \mathrm{Sm}\right]_{2}\left(\mu-\eta^{2}: \eta^{2}-\mathrm{N}_{2}\right)(\mathbf{6 7})} \\
{[105]}\end{array}$ & $1.08(3)$ & $\begin{array}{l}2.880(18) \\
2.974(18)\end{array}$ & - \\
\hline $\begin{array}{c}{\left[\left(\mathrm{Li}(\text { thf })_{2}\right]\left[\left({ }^{\mathrm{Cy}} \text { calix }[4] \text { pyrrole }\right)_{2} \mathrm{Sm}_{3} \mathrm{Li}_{2}\right]\left(\mu_{5}-\eta^{1}: \eta^{1}: \eta^{2}: \eta^{2}: \eta^{2}-\mathrm{N}_{2}\right)\right.} \\
(\mathbf{6 8})[105]\end{array}$ & $1.502(5)$ & $\begin{array}{c}2.249(4) \\
(\mathrm{Sm}(1)-\mathrm{N}(1)) \\
2.253(4) \\
(\mathrm{Sm}(1)-\mathrm{N}(1)) \\
2.355(4) \\
(\mathrm{Sm}(2)-\mathrm{N}(1)) \\
2.370(4) \\
(\mathrm{Sm}(2)-\mathrm{N}(1)) \\
2.398(4) \\
(\mathrm{Sm}(3)-\mathrm{N}(1)) \\
2.376(4) \\
(\mathrm{Sm}(3)-\mathrm{N}(1))\end{array}$ & - \\
\hline
\end{tabular}

To date, all examples of complexes containing an $\mathrm{N}_{2}{ }^{4-}$ ligand, derived from dinitrogen activation by a rare earth metal centre involve multidentate ligands (Figure 10) [101-106]. The first example of a structurally characterised rare earth complex containing an $\mathrm{N}_{2}{ }^{4-}$ ligand was reported by Gambarotta and co-workers [101]. The octametallic complex $\left[\mathrm{Li}(\mathrm{thf})_{2}\right]_{2}\left[\left({ }^{\mathrm{Et} 2}\right.\right.$ calix[4]pyrrole $) \mathrm{Sm}_{2}\left(\mathrm{~N}_{2} \mathrm{Li}{ }_{4}\right)(\mathbf{6 1})$ was prepared by reaction of $\mathrm{SmCl}_{3}(\text { thf })_{3}$ with $[\mathrm{Li}(\mathrm{thf})]_{4}\left[{ }^{\mathrm{Et} 2}\right.$ calix[4]pyrrole] and subsequent reduction with $\mathrm{Li}$ metal under an argon atmosphere, followed by exposure to $\mathrm{N}_{2}$. The reaction by-products were not determined. In the solid state structure, there is an octahedron of metal ions coordinating to the $\mathrm{N}_{2}{ }^{4-}$ ligand which is $\eta^{2}$-bound to the two $\mathrm{Sm}^{\mathrm{III}}$ in the apical sites, and both $\eta^{2}$ - and $\eta^{1}$-bound to the opposite pairs of the four $\mathrm{Li}^{+}$ions in the equatorial plane. The $\mathrm{N}-\mathrm{N}$ distance of 1.525(4) $\AA$, combined with a total magnetic moment of $2.72 \mu \mathrm{B}$, is consistent with formulation of the complex as $\left[\mathrm{Sm}^{\mathrm{III}}\right]_{2}\left(\mathrm{~N}_{2}{ }^{4-}\right)$. 


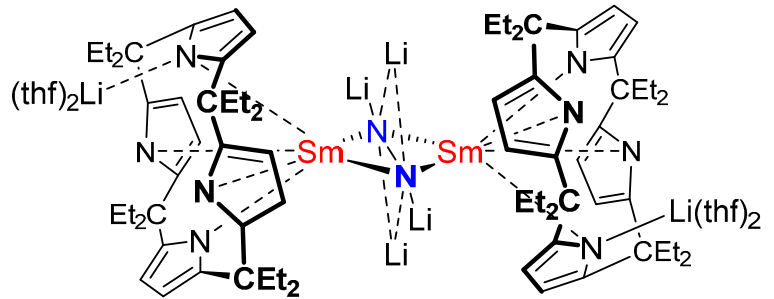

(61)

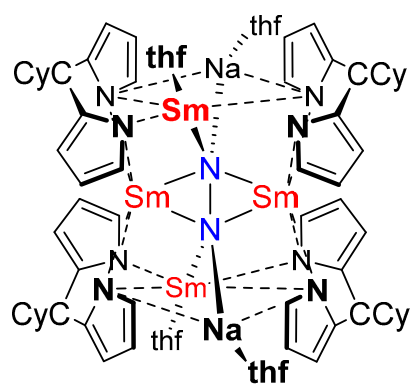

(66)

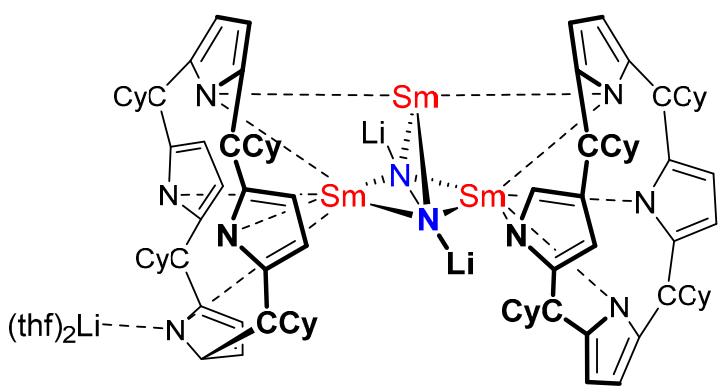

(68)

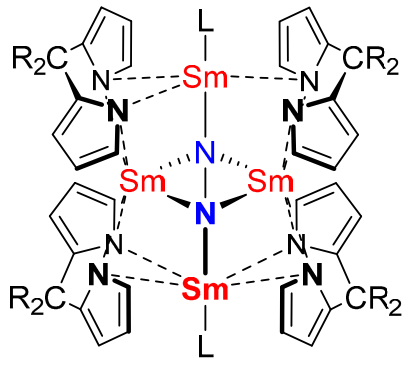

$\mathrm{CR}_{2}=$

$$
\mathrm{L}=\text { thf } \quad \mathrm{CPh}_{2}(62)
$$

CCy $(63)$

$\mathrm{CEt}_{2}(64)$

$\mathrm{L}=\mathrm{dme} \quad \mathrm{C}(\mathrm{Me}) \mathrm{Ph}(\mathbf{6 5})$

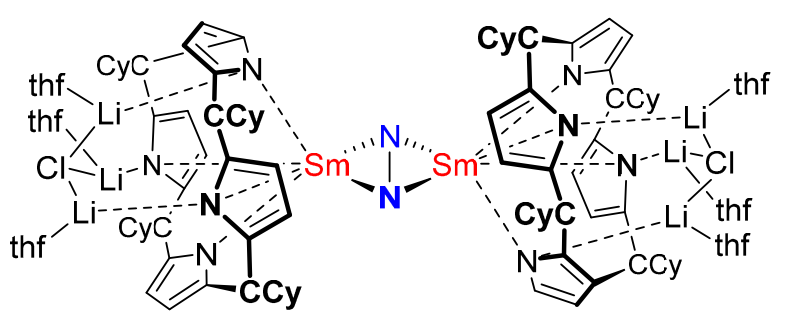

(67)

Figure 10. Lanthanide complexes with multidentate donors resulting from $\mathrm{N}_{2}$ activation to $\mathrm{N}_{2}{ }^{4-}$.

A family of tetrametallic Sm dipyrrolide complexes $\left[\left\{\mathrm{R}_{2} \mathrm{C}\left(\mathrm{C}_{4} \mathrm{H}_{3} \mathrm{~N}\right)_{2}\right\} \operatorname{Sm}(\mathrm{L})\right]_{4}\left(\mu_{4}-\eta^{1}: \eta^{1}: \eta^{2}: \eta^{2}-\mathrm{N}_{2}\right)$ $\left(\mathrm{L}=\right.$ thf, $\left.\mathrm{CR}_{2}=\mathrm{CPh}_{2}(\mathbf{6 2}), \mathrm{CCy}(\mathbf{6 3}), \mathrm{CEt}_{2}(\mathbf{6 4}) ; \mathrm{L}=\mathrm{dme}, \mathrm{CR}_{2}=\mathrm{C}(\mathrm{Me}) \mathrm{Ph}(\mathbf{6 5})\right)$ was prepared by reaction of $\mathrm{SmI}_{2}(\mathrm{thf})_{2}$ with the corresponding alkali metal dipyrrolide salt, and display both side-on and end-on $\mathrm{N}_{2}$ coordination [102-104]. 62-65 were stable both thermally and in vacuo. All compounds were structurally characterised using X-ray diffraction experiments; the $\mathrm{N}-\mathrm{N}$ bond lengths range from $1.392(16)$ to $1.42(2) \AA$, and variable coordination modes of the pyrrolide ligands are observed across all complexes. Reduction of $\mathbf{6 3}$ with $\mathrm{Na}$ afforded $[\mathrm{Na}(\text { thf })]_{2}\left[\left\{\mathrm{R}_{2} \mathrm{C}\left(\mathrm{C}_{4} \mathrm{H}_{3} \mathrm{~N}\right)_{2}\right\} \mathrm{Sm}(\mathrm{thf})\right]_{4}\left(\mu_{6}-\eta^{1}: \eta^{1}: \eta^{1}: \eta^{1}: \eta^{2}: \eta^{2}-\mathrm{N}_{2}\right)(66)$. The $\mathrm{N}_{2}{ }^{4-}$ ligand is bound end-on to two $\mathrm{Sm}$ ions and side-on to the other two, as well as being end-on bound to two $\mathrm{Na}^{+}$ions. Assignment of the reduction level of dinitrogen to $\mathrm{N}_{2}{ }^{4-}$ leads to a formal oxidation state of +2.5 for each samarium centre. This is proposed on the basis of the N-N bond distance (1.371(16) $\AA$; slightly shorter than that expected for an $\mathrm{N}-\mathrm{N}$ single bond), magnetic moment (4.05 $\mu_{\mathrm{B}}$; lower than the analogous divalent complex 63) and short Sm-Sm contacts in the solid state which may promote magnetic couplings. 
Tetra-calix-pyrrole complexes $\left[\{\mathrm{Li}(\mathrm{thf})\}_{3}\left(\mu_{3}-\mathrm{Cl}\right)\right]\left[\left({ }^{\mathrm{Cy}} \text { calix }[4] \text { pyrrole }\right) \operatorname{Sm}\right]_{2}\left(\mu-\eta^{2}: \eta^{2}-\mathrm{N}_{2}\right)(67)$ and the unusual trimetallic [Li(thf) $\left.)_{2}\right]\left[{ }^{C y}\right.$ calix[4]pyrrole) $\left.{ }_{2} \mathrm{Sm}_{3} \mathrm{Li}_{2}\right]\left(\mu 5-\eta^{1}: \eta^{1}: \eta^{2}: \eta^{2}: \eta^{2}-\mathrm{N}_{2}\right)$ (68) contain formal $\mathrm{N}_{2}{ }^{4-}$ ligands, on the basis of charge neutrality implied from the presence of $\mathrm{Sm}^{\mathrm{III}}$ centres but have

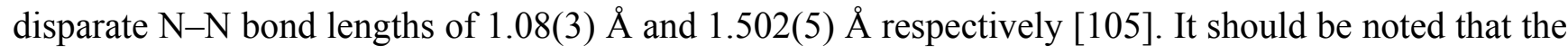
crystallographic data for $\mathbf{6 7}$ was only of a good enough quality to obtain structural connectivity.

\section{Dinitrogen Activation by Actinide Complexes}

\subsection{Complexes Containing an Activated $N_{2}$ Ligand}

Perhaps surprisingly, given the number of examples of dinitrogen activation by rare earth complexes, there are very few examples of $\mathrm{N}_{2}$ activation with actinide complexes despite the presence of uranium in early catalysts for the Haber-Bosch process (Figure 11) [107]. Actinide-element bonding in the model system $\left[\mathrm{X}_{3} \mathrm{An}\right]_{2}\left(\mu-\eta^{2}: \eta^{2}-\mathrm{N}_{2}\right)(\mathrm{An}=\mathrm{Th}-\mathrm{Pu}, \mathrm{X}=\mathrm{F}, \mathrm{Cl}, \mathrm{Br}, \mathrm{Me}, \mathrm{H}, \mathrm{OPh})$ has recently been studied using relativistic DFT calculations [108].

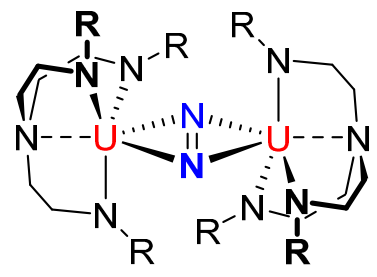

(69)

$\mathrm{R}=\mathrm{SiMe}_{2}{ }^{\mathrm{t}} \mathrm{Bu}$

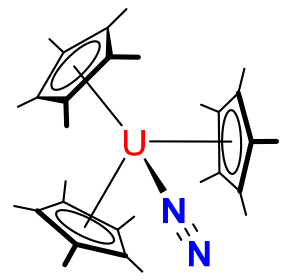

(73)

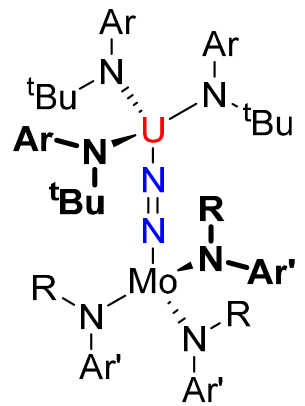

$\mathrm{Ar}=3,5-\mathrm{Me}-\mathrm{C}_{6} \mathrm{H}_{3}$

$\mathrm{Ar} \mathrm{r}^{\prime}=\mathrm{Ph}, \quad \mathrm{R}={ }^{\mathrm{t}} \mathrm{Bu}(\mathbf{7 0})$

$A r^{\prime}=A r, \quad R=A d(71)$

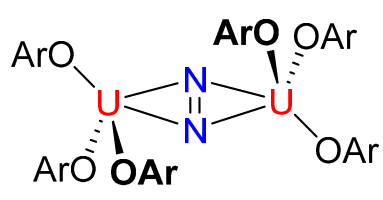

$\mathrm{Ar}=2,6-{ }^{\mathrm{t}} \mathrm{Bu}-\mathrm{C}_{6} \mathrm{H}_{3} \quad(\mathbf{7 4})$

$2,4,6-{ }^{\mathrm{t}} \mathrm{Bu}-\mathrm{C}_{6} \mathrm{H}_{2}(\mathbf{7 5})$

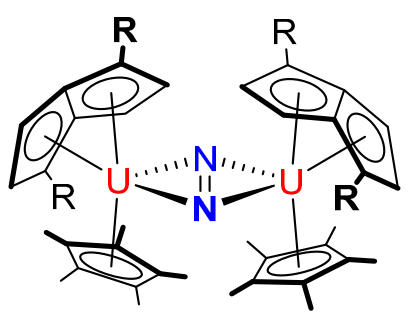

$\mathrm{R}=\mathrm{Si}^{\mathrm{i}} \mathrm{Pr}_{3}$

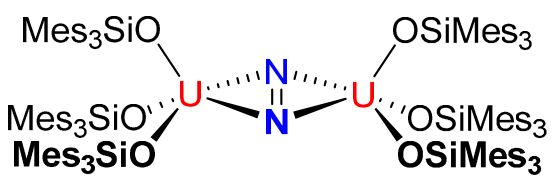

(76-Eclipsed)

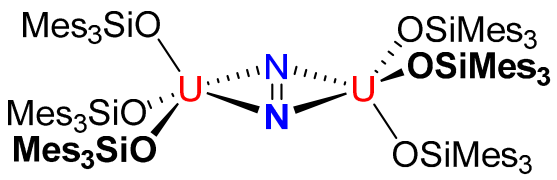

(76-Staggered)

Mes $=2,4,6-\mathrm{Me}-\mathrm{C}_{6} \mathrm{H}_{2}$

Figure 11. Actinide complexes resulting from $\mathrm{N}_{2}$ activation (no $\mathrm{N}-\mathrm{N}$ bond cleavage).

For reference, $\mathrm{N}-\mathrm{N}$ bond lengths obtained from single crystal $\mathrm{X}$-ray diffraction experiments, $\mathrm{N}-\mathrm{N}$ stretching frequencies (obtained by IR or Raman spectroscopy) and ${ }^{14 / 15} \mathrm{~N}-\mathrm{NMR}$ spectroscopic data of actinide $\mathrm{N}_{2}$ complexes are summarised in Table 4. 
Table 4. Summary of actinide $\mathrm{N}_{2}$ complexes.

\begin{tabular}{|c|c|c|c|c|}
\hline Complex (\#) [Reference] & Stability & $\begin{array}{l}\text { N-N Bond } \\
\text { Length }(\AA)\end{array}$ & $\begin{array}{c}\mathrm{N}-\mathrm{N} \\
\text { Frequency } \\
\left(\mathrm{cm}^{-1}\right)\end{array}$ & $\begin{array}{c}{ }^{14 / 15} \mathrm{~N}-\mathrm{NMR} \\
\text { Spectroscopy } \\
(\mathrm{ppm})^{\mathrm{a}} \\
\end{array}$ \\
\hline $\mathrm{N}_{2}$ & - & $1.0975[66]$ & $2331[67]$ & $-75[68]$ \\
\hline$\left[\left\{\mathrm{N}\left(\mathrm{CH}_{2} \mathrm{CH}_{2} \mathrm{NSiMe}_{2}{ }^{t} \mathrm{Bu}\right)_{3}\right\} \mathrm{U}\right]_{2}\left(\mu-\eta^{2}: \eta^{2}-\mathrm{N}_{2}\right)(\mathbf{6 9})$ & $\begin{array}{l}\text { Stable under } \mathrm{N}_{2} \\
(1 \mathrm{~atm})\end{array}$ & \multirow{2}{*}{$1.109(7)$} & \multirow{2}{*}{-} & \multirow{2}{*}{-} \\
\hline$[109]$ & $\begin{array}{l}\mathrm{N}_{2} \text { dissociation } \\
\text { in vacuo }\end{array}$ & & & \\
\hline \multirow{2}{*}{$\begin{array}{c}\left\{\mathrm{Ph}\left({ }^{t} \mathrm{Bu}\right) \mathrm{N}\right\}_{3} \mathrm{Mo}\left(\mu_{2}-\eta^{1}: \eta^{1}-\mathrm{N}_{2}\right) \mathrm{U}\left\{\mathrm{N}\left({ }^{t} \mathrm{Bu}\right)(3,5-\mathrm{Me}-\right. \\
\left.\left.\mathrm{C}_{6} \mathrm{H}_{3}\right)\right\}_{3}(\mathbf{7 0})[110]\end{array}$} & $\begin{array}{l}\text { Stable in vacuo at } \\
255^{\circ} \mathrm{C}\end{array}$ & \multirow[t]{2}{*}{$1.232(11)$} & $-\left({ }^{14} \mathrm{~N}_{2}\right)$ & \multirow[t]{2}{*}{-} \\
\hline & "Thermally stable" & & $1547\left({ }^{15} \mathrm{~N}_{2}\right)$ & \\
\hline \multirow{2}{*}{$\begin{array}{c}\left\{\left(3,5-\mathrm{Me}-\mathrm{C}_{6} \mathrm{H}_{3}\right)(\mathrm{Ad}) \mathrm{N}\right\}_{3} \mathrm{Mo}\left(\mu_{2}-\eta^{1}: \eta^{1}-\mathrm{N}_{2}\right) \\
\mathrm{U}\left\{\mathrm{N}\left({ }^{t} \mathrm{Bu}\right)\left(3,5-\mathrm{Me}-\mathrm{C}_{6} \mathrm{H}_{3}\right)\right\}_{3}(\mathbf{7 1})[110]\end{array}$} & $\begin{array}{l}\text { Stable in vacuo at } \\
25{ }^{\circ} \mathrm{C} \\
\end{array}$ & \multirow[t]{2}{*}{$1.23(2)$} & $1568\left({ }^{14} \mathrm{~N}_{2}\right)$ & \multirow[t]{2}{*}{-} \\
\hline & "Thermally stable" & & $1527\left({ }^{15} \mathrm{~N}_{2}\right)$ & \\
\hline \multirow{2}{*}{$\begin{array}{c}{\left[\left(\eta^{5}-\mathrm{C}_{5} \mathrm{Me}_{5}\right)\left(\eta^{8}-1,4-\mathrm{Si}^{i} \mathrm{Pr}_{3}-\mathrm{C}_{8} \mathrm{H}_{4}\right) \mathrm{U}_{2}\left(\mu-\eta^{2}: \eta^{2}-\mathrm{N}_{2}\right)\right.} \\
\text { (72) }[111]\end{array}$} & $\begin{array}{c}75 \% \text { conversion to } 72 \\
\text { at } 50 \mathrm{psi} \mathrm{N}_{2}\end{array}$ & \multirow[b]{2}{*}{$1.232(10)$} & \multirow[b]{2}{*}{-} & \multirow[b]{2}{*}{-} \\
\hline & $\begin{array}{c}\mathrm{N}_{2} \text { dissociation } \\
\text { in vacuo, in solution } \\
\text { and solid state }\end{array}$ & & & \\
\hline \multirow{3}{*}{$\left(\eta^{5}-\mathrm{C}_{5} \mathrm{Me}_{5}\right)_{3} \mathrm{U}\left(\eta^{1}-\mathrm{N}_{2}\right)(\mathbf{7 3})[112]$} & $\begin{array}{c}\text { Crystallisation at } \\
80 \mathrm{psi} \mathrm{N}_{2}\end{array}$ & \multirow{3}{*}{$1.120(14)$} & $2207\left({ }^{14} \mathrm{~N}_{2}\right)$ & \multirow{3}{*}{-} \\
\hline & $\mathrm{N}_{2}$ dissociation & & & \\
\hline & $\begin{array}{c}\text { in vacuo or in solution } \\
\text { under } \mathrm{N}_{2}(1 \mathrm{~atm})\end{array}$ & & $2134\left({ }^{15} \mathrm{~N}_{2}\right)$ & \\
\hline \multirow{3}{*}[(2,6-{}^{-}\mathrm{Bu}-\mathrm{C}_{6}\mathrm{H}_{3}\mathrm{O})_{3}\mathrm{U}]{$_{2}\left(\mu-\eta^{2}: \eta^{2}-\mathrm{N}_{2}\right)(\mathbf{7 4})[88]$} & $\mathrm{N}_{2}$ dissociation & $1.163(19)$ & & \multirow{3}{*}{-} \\
\hline & in vacuo and in & $1.204(17)$ & - & \\
\hline & solution at $25^{\circ} \mathrm{C}$ & $1.201(19)$ & & \\
\hline \multirow{2}{*}[(2,4,6-{}^{t}\mathrm{Bu}-\mathrm{C}_{6}\mathrm{H}_{2}\mathrm{O})_{3}\mathrm{U}]{$_{2}\left(\mu-\eta^{2}: \eta^{2}-\mathrm{N}_{2}\right)(75)[88]$} & $\begin{array}{c}\text { Stable in vacuo } \\
\text { at } 25^{\circ} \mathrm{C} \\
\end{array}$ & \multirow{2}{*}{$1.236(5)$} & $1451\left({ }^{14} \mathrm{~N}_{2}\right)$ & \multirow{2}{*}{ - } \\
\hline & $\begin{array}{l}\mathrm{N}_{2} \text { dissociation at } \\
80^{\circ} \mathrm{C} \text { in solution }\end{array}$ & & $1404\left({ }^{15} \mathrm{~N}_{2}\right)$ & \\
\hline \multirow[b]{2}{*}[\{(\mathrm{Mes})_{3}\mathrm{SiO}\}_{3}\mathrm{U}]{$_{2}\left(\mu-\eta^{2}: \eta^{2}-\mathrm{N}_{2}\right)(76)[87]$} & $\begin{array}{l}\text { Stable in vacuo at } \\
225^{\circ} \mathrm{C} \\
\end{array}$ & $\begin{array}{l}1.124(12) \\
\text { (eclipsed) }\end{array}$ & $1437\left({ }^{14} \mathrm{~N}_{2}\right)$ & \multirow[b]{2}{*}{4213.5} \\
\hline & $\begin{array}{c}\text { Slowly forms } \\
\mathrm{U}\left\{\mathrm{OSi}(\mathrm{Mes})_{3}\right\}_{4} \text { at } \\
100^{\circ} \mathrm{C} \text { in solution }\end{array}$ & $\begin{array}{c}1.080(11) \\
\text { (staggered) }\end{array}$ & $1372\left({ }^{15} \mathrm{~N}_{2}\right)$ & \\
\hline
\end{tabular}

${ }^{\text {a }}$ Referenced to $\mathrm{CH}_{3}{ }^{15} \mathrm{NO}$.

The first example of dinitrogen activation by an actinide complex was reported by Scott and co-workers; the trivalent uranium complex $\left\{\mathrm{N}\left(\mathrm{CH}_{2} \mathrm{CH}_{2} \mathrm{NSiMe}_{2}{ }^{t} \mathrm{Bu}\right)_{3}\right\} \mathrm{U}$ reacts with $\mathrm{N}_{2}(1 \mathrm{~atm})$ to yield $\left[\left\{\mathrm{N}\left(\mathrm{CH}_{2} \mathrm{CH}_{2} \mathrm{NSiMe}_{2}{ }^{t} \mathrm{Bu}\right)_{3}\right\} \mathrm{U}_{2}\left(\mu-\eta^{2}: \eta^{2}-\mathrm{N}_{2}\right)(69)\right.$ [109]. In solution, the reaction is reversible and 69 converts back to the trivalent uranium starting material when freeze-pump-thaw degassed (Scheme 1). The solid state structure of 69 illustrates the side-on binding mode of $\mathrm{N}_{2}$ and features an $\mathrm{N}-\mathrm{N}$ bond 
length of 1.109(7) A. Alongside solution magnetic susceptibility measurements of $3.22 \mu$ в per uranium centre, these data agree with the dimer being formulated as $\left[\mathrm{U}^{\mathrm{III}}\right]_{2}\left(\mathrm{~N}_{2}{ }^{0}\right)$.
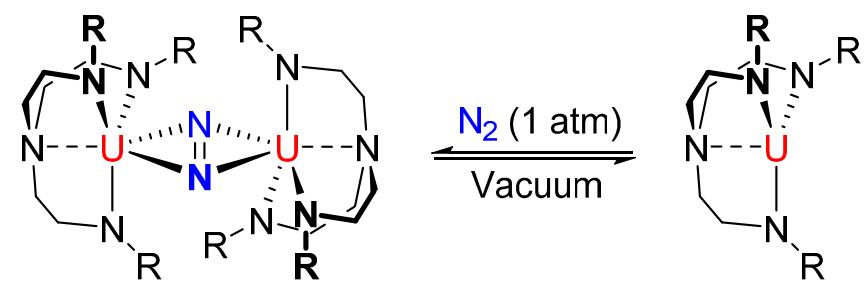

(69)

$\mathrm{R}=\mathrm{SiMe}_{2}{ }^{\mathrm{t}} \mathrm{Bu}$

Scheme 1. Reversible $\mathrm{N}_{2}$ binding in 69.

Cummins and co-workers reported thermally stable heterobimetallic $\mathrm{U}-\mathrm{Mo} \mathrm{N}_{2}$ complexes featuring the end-on binding mode of $\mathrm{N}_{2}$; the $\mathrm{U}^{\mathrm{III}}$ tris(amide) $\mathrm{U}\left\{\mathrm{N}\left({ }^{t} \mathrm{Bu}\right) \operatorname{Ar}\right\}_{3}($ thf $)\left(\mathrm{Ar}=3,5-\mathrm{Me}-\mathrm{C}_{6} \mathrm{H}_{3}\right)$ reacts with $\operatorname{Mo}\left\{\mathrm{N}(\mathrm{R}) \mathrm{Ar}^{\prime}\right\}_{3}$ under an $\mathrm{N}_{2}$ atmosphere $(1$ atm $)$ to yield $\left\{\mathrm{Ar}^{\prime}(\mathrm{R}) \mathrm{N}\right\}_{3} \operatorname{Mo}\left(\mu_{2}-\eta^{1}: \eta^{1}-\right.$ $\left.\mathrm{N}_{2}\right) \mathrm{U}\left\{\mathrm{N}\left({ }^{t} \mathrm{Bu}\right) \mathrm{Ar}_{3}\left(\mathrm{R}={ }^{t} \mathrm{Bu}, \mathrm{Ar}^{\prime}=\mathrm{Ph}(\mathbf{7 0}) ; \mathrm{R}=\mathrm{Ad}, \mathrm{Ar}^{\prime}=3,5-\mathrm{Me}-\mathrm{C}_{6} \mathrm{H}_{3}\right.\right.$ (71)) [110]. The solid state molecular structure of $\mathbf{7 0}$ shows an $\mathrm{N}-\mathrm{N}$ bond distance of $1.232(11) \AA$, consistent with $\mathrm{N}_{2}{ }^{2-}$ and a formal oxidation state of $\mathrm{U}^{\mathrm{IV}}$. In principal, $\mathrm{U}^{\mathrm{III}}$ and $\mathrm{Mo}^{\mathrm{III}}$ metal centres can provide the 6 electrons required for $\mathrm{N}_{2}$ bond cleavage and the stability of $\mathbf{7 0}$ and $\mathbf{7 1}$ should be noted with reference to $\operatorname{Mo}\left\{\mathrm{N}\left({ }^{t} \mathrm{Bu}\right) \mathrm{Ar}^{\prime}\right\}_{3}$ which cleaves $\mathrm{N}_{2}$ and forms a terminal nitride $(\mathrm{Mo} \equiv \mathrm{N})$ under mild conditions [113-115].

Reversible side-on binding and $\mathrm{N}_{2}$ activation was demonstrated by Cloke et al., using a mixed sandwich $U^{\mathrm{III}}$ pentalene complex. $\left(\eta^{5}-\mathrm{C}_{5} \mathrm{Me}_{5}\right)\left(\eta^{8}-1,4-\mathrm{Si}^{i} \mathrm{Pr}_{3}-\mathrm{C}_{8} \mathrm{H}_{4}\right) \mathrm{U}$ reacts with $\mathrm{N}_{2}$ to yield $\left[\left(\eta^{5}-\right.\right.$ $\left.\mathrm{C}_{5} \mathrm{Me}_{5}\right)\left(\eta^{8}-1,4-\mathrm{Si}^{i} \mathrm{Pr}_{3}-\mathrm{C}_{8} \mathrm{H}_{4}\right) \mathrm{U}_{2}\left(\mu-\eta^{2}: \eta^{2}-\mathrm{N}_{2}\right)(72)$ [111]. The solid state structure features an $\mathrm{N}-\mathrm{N}$ bond length of 1.232(10) $\AA$ which is consistent with reduction to $\mathrm{N}_{2}{ }^{2-}$; regardless of the formal reduction level, the relief of steric crowding in $\mathbf{7 2}$ likely drives the facile loss of $\mathrm{N}_{2}$ when there is no overpressure.

To date, the only example of a monometallic f-element complex of $\mathrm{N}_{2}$ was reported by Evans et al.; sterically crowded $\mathrm{U}\left(\eta^{5}-\mathrm{C}_{5} \mathrm{Me}_{5}\right)_{3}$ reacts with $\mathrm{N}_{2}(80 \mathrm{psi})$ to afford $\left(\eta^{5}-\mathrm{C}_{5} \mathrm{Me}_{5}\right)_{3} \mathrm{U}\left(\eta^{1}-\mathrm{N}_{2}\right)$ (73) [112]. $\mathrm{N}_{2}$ binding is reversible and lowering the pressure results in $\mathrm{N}_{2}$ dissociation. The solid state structure of 73 shows the $\mathrm{N}_{2}$ ligand is bound end-on and linearly $\left(\mathrm{U}-\mathrm{N}-\mathrm{N}=180^{\circ}\right)$ and the $\mathrm{N}-\mathrm{N}$ distance is $1.120(14) \AA$ which is statistically equivalent with free $\mathrm{N}_{2}$.

Arnold and co-workers reported that the trivalent uranium aryloxides $\mathrm{U}(\mathrm{OAr})_{3}\left(\mathrm{Ar}=2,6-{ }^{t} \mathrm{Bu}-\mathrm{C}_{6} \mathrm{H}_{3}\right.$ or $\left.2,4,6-{ }^{t} \mathrm{Bu}-\mathrm{C}_{6} \mathrm{H}_{2}\right)$ bind $\mathrm{N}_{2}(1 \mathrm{~atm})$ to form the side-on bound $\mathrm{N}_{2}$ adducts $\left[(\mathrm{ArO})_{3} \mathrm{U}_{2}\left(\mu-\eta^{2}: \eta^{2}-\mathrm{N}_{2}\right)(74\right.$ and $\mathbf{7 5}$ respectively) [88]. Though $\mathbf{7 4}$ was obtained as a minor product, the more sterically hindered $\mathbf{7 5}$ was formed in quantitative yield and was stable under dynamic vacuum and in the presence of coordinating solvents and polar small molecules $\left(\mathrm{CO}\right.$ and $\left.\mathrm{CO}_{2}\right)$ under ambient conditions. $\mathrm{N}_{2}$ loss was observed when 75 was heated to $80{ }^{\circ} \mathrm{C}$ in a toluene solution. The solid state $\mathrm{N}-\mathrm{N}$ bond lengths are 1.163(19), 1.204(17), 1.201(19) $\AA$ (74) and 1.236(5) $\AA$ (75) which indicate significant $\mathrm{N}_{2}$ reduction by the electron rich $U^{\mathrm{III}}$ metal centres. Consistent with this, Raman spectroscopy performed on $\mathbf{7 5}$ showed a strong band at $1451 \mathrm{~cm}^{-1}$ for the $\mathrm{N}-\mathrm{N}$ stretch $\left(1404 \mathrm{~cm}^{-1}\right.$ in the $\left.\mathbf{7 5}^{-15} \mathbf{N}_{2}\right)$ which is significantly lower than in free $\mathrm{N}_{2}\left(2331 \mathrm{~cm}^{-1}\right)$ [67]. DFT calculations indicate a ${ }^{5} \mathrm{Ag}$ ground state which agrees with the $\left[\mathrm{U}^{\mathrm{IV}}\right]_{2}\left(\mathrm{~N}_{2}{ }^{2-}\right)$ description of 75. Significantly, the $\mathrm{U}-\mathrm{N}\left(\mathrm{N}_{2}\right)$ interaction derives from two occupied MOs 
showing $\pi$ backbonding from uranium $\mathrm{f}$ orbitals into an $\mathrm{N}_{2}$ antibonding $\pi_{\mathrm{g}}$ orbitals and that the interaction is strongly polarised. The bonding description is very similar to that in previously calculated models for 69 (formally $\left.\mathrm{N}_{2}{ }^{0}\right)$ [116,117] and $72\left(\mathrm{~N}_{2}{ }^{2-}\right)$ [118] which display very different $\mathrm{N}-\mathrm{N}$ bond lengths. While experimental $\mathrm{N}-\mathrm{N}$ bond distances determined by $\mathrm{X}$-ray diffraction experiments are undeniably useful for quick comparisons, the bond length is likely underestimated since the data is based on electron density rather than atomic positions and thus may not reflect the level of dinitrogen reduction. In these studies, $\mathrm{N}-\mathrm{N}$ stretching wavenumbers were more accurately reproduced by calculation than bond length and it is proposed that this would be a more suitable measurement for probing $\mathrm{N}_{2}$ reduction.

The most robust actinide $\mathrm{N}_{2}$ complex prepared to date is $\left[\left\{(\mathrm{Mes})_{3} \mathrm{SiO}\right\}_{3} \mathrm{U}\right]_{2}\left(\mu-\eta^{2}: \eta^{2}-\mathrm{N}_{2}\right)$ (76) (Mes $=2,4,6-\mathrm{Me}-\mathrm{C}_{6} \mathrm{H}_{2}$ ) and is stable both in vacuo and in toluene solution up to $100{ }^{\circ} \mathrm{C}$, at which point $\mathrm{U}\left\{\mathrm{OSi}(\mathrm{Mes})_{3}\right\}_{4}$ is slowly formed as the major product $(52 \%$ conversion after $18 \mathrm{~h})$ [87]. 76 is isolated from the reaction of $\mathrm{U}\left\{\mathrm{N}\left(\mathrm{SiMe}_{3}\right)_{2}\right\}_{3}$ with 3 equivalents of $\mathrm{HOSi}(\mathrm{Mes})_{3}$ under an $\mathrm{N}_{2}$ atmosphere (1 atm). Raman spectroscopy shows a peak at $1437 \mathrm{~cm}^{-1}$ assigned to the $\mathrm{N}-\mathrm{N}$ stretching mode, indicating a significant level of reduction with respect to free dinitrogen $\left(2331 \mathrm{~cm}^{-1}\right)$ and comparing well with $1451 \mathrm{~cm}^{-1}$ recorded for 75 where reduction to $\mathrm{N}_{2}{ }^{2-}$ was assigned. The N-N distances in the solid state are 1.124(12) $\AA$ (76-Eclipsed) and 1.080(11) $\AA$ (76-Staggered), which are statistically equivalent to that of free $\mathrm{N}_{2}$; the disparity in implied reduction of $\mathrm{N}_{2}$ from Raman spectroscopy and X-ray diffraction experiments again highlighting that the latter may not be best suited for assigning reduction in these systems.

\subsection{Complexes Resulting from $\mathrm{N}_{2}$ Cleavage}

Tetra-calix-pyrrole ligands bound to $\mathrm{Sm}^{\mathrm{II}}$ centres have been demonstrated to activate $\mathrm{N}_{2}$ by Gambarotta and co-workers [101]. With $\mathrm{U}^{\mathrm{III}}$, an unprecedented example of $\mathrm{N}-\mathrm{N}$ bond cleavage using an molecular f-element complex was observed; when $[\mathrm{K}(\mathrm{dme})]\left[\left({ }^{\mathrm{E} t 2}\right.\right.$ calix $[4]$ pyrrole $\left.) \mathrm{U}(\mathrm{dme})\right]$ is treated with potassium naphthalenide under an atmosphere of $\mathrm{N}_{2}, \mathrm{~N}-\mathrm{N}$ bond cleavage occurs to afford $[\mathrm{K}(\mathrm{dme}) 4]\left[\left\{\mathrm{K}(\mathrm{dme})\left({ }^{\mathrm{Et} 2} \text { calix[4]pyrrole }\right) \mathrm{U}\right\}_{2}(\mu-\mathrm{NK})_{2}\right]$ (77) (Scheme 2) [119]. 77 contains two bridging nitrides (U-N: 2.076(6) and 2.099(5) $\AA$ ) which have contacts with potassium ions (N-K: 2.554(6) $\AA$ ) that bridge two pyrrolide units on separate ligands. It was postulated that 77 is a Class $1 \mathrm{U}^{\mathrm{IV}}-\mathrm{U}^{\mathrm{V}}$ mixed valence complex on the basis of an absorption at $1247 \mathrm{~nm}$ in the near-IR spectrum which is characteristic of $\mathrm{U}^{\mathrm{V}}$. The paramagnetism of 77 resulted in NMR silence in both ${ }^{15} \mathrm{~N}$ - and ${ }^{14} \mathrm{~N}-\mathrm{NMR}$ spectra. 


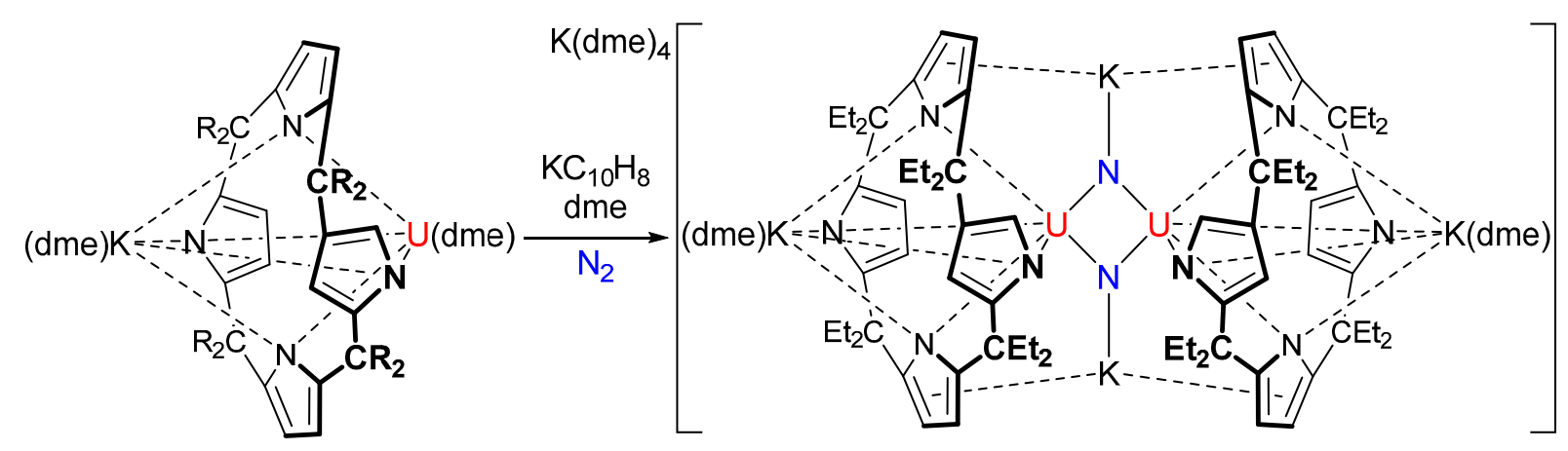

(77)

Scheme 2. N-N bond cleavage using a $\mathrm{U}^{\mathrm{III}}$ complex to form $\mathbf{7 1 .}$

Reduction of the thorium bisphenolate complex $\left[\mathrm{K}(\mathrm{dme})_{2}\right]\left[\left\{\left(2-{ }^{t} \mathrm{Bu}-4-\mathrm{Me}-\mathrm{C}_{6} \mathrm{H}_{2} \mathrm{O}\right)_{2}-6-\right.\right.$ $\left.\left.\mathrm{CH}_{2}\right\}_{2} \mathrm{ThCl}(\mathrm{dme})\right]$ (A) with potassium naphthalenide under an atmosphere of dinitrogen unexpectedly resulted in the amide complex $[\mathrm{K}(\mathrm{dme}) 4]\left[\left\{\left(2-{ }^{t} \mathrm{Bu}-4-\mathrm{Me}-\mathrm{C}_{6} \mathrm{H}_{2} \mathrm{O}\right)_{2}-6-\mathrm{CH}_{2}\right\}_{2} \mathrm{Th}\left(\mathrm{NH}_{2}\right)(\mathrm{dme})\right] 78$ which is the first example of $\mathrm{N}_{2}$ functionalisation using an f-element complex (Scheme 3). The parent $\left[\mathrm{NH}_{2}\right]^{-}$ amide ligand is confirmed through ${ }^{15} \mathrm{~N}-\mathrm{NMR}$ spectroscopy which shows a triplet at $155.01 \mathrm{ppm}$ $\left({ }^{1} J_{\mathrm{NH}}=57.2 \mathrm{~Hz}\right)[120]$. The proposed mechanism of this transformation involves formation of a formally zero-valent thorium intermediate which contains two bound $\left[\mathrm{C}_{10} \mathrm{H}_{8} \mathrm{~K}(18 \mathrm{c} 6)\right]$ fragments (identified through a single crystal X-ray diffraction experiment). This intermediate can then react with the starting material A leading to $\mathrm{N}_{2}$ activation, cleavage and hydrogenation as a result of $\mathrm{H}$ atom abstraction from solvent molecules.

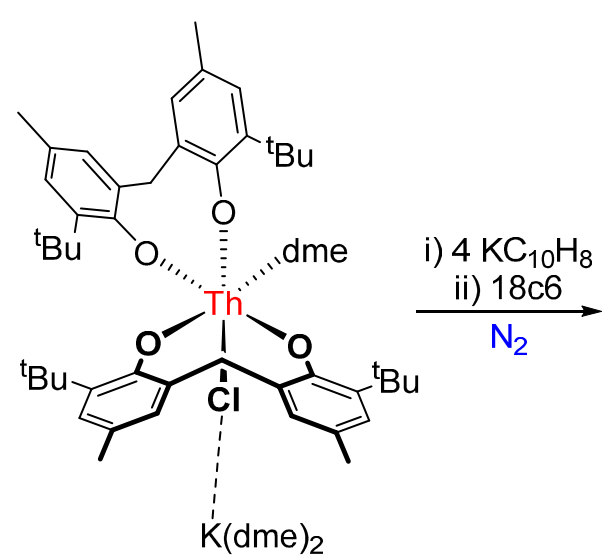

A

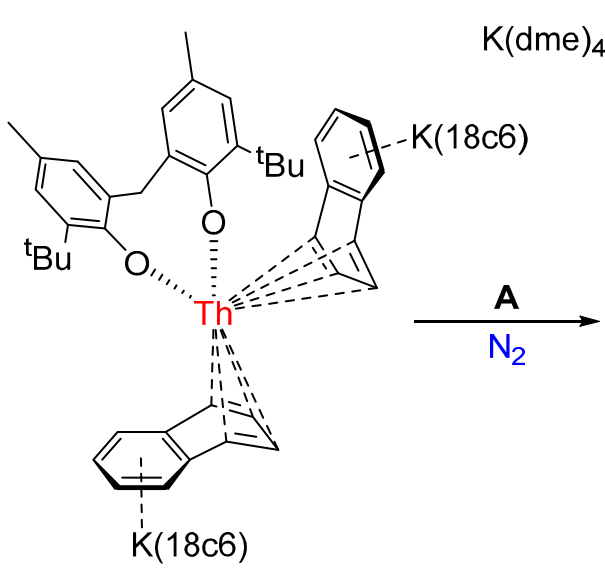

$\mathrm{K}(18 \mathrm{c} 6)$

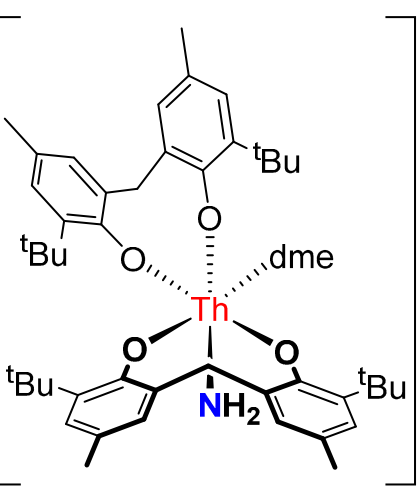

(78)

$\mathrm{KC}_{10} \mathrm{H}_{18}$

Scheme 3. $\mathrm{N}_{2}$ cleavage and hydrogenation by a thorium complex.

\section{White Phosphorus Activation by Rare Earth Complexes}

Rare earth complexes resulting from $\mathrm{P}_{4}$ activation are illustrated in Figure 12. For reference, average P-P and Ln-P bond lengths obtained from single crystal X-ray diffraction experiments, and ${ }^{31} \mathrm{P}-\mathrm{NMR}$ spectroscopic resonances are summarised in Table 5. The structural cores of complexes 79-84 are shown in Figure 13 for clarity. 


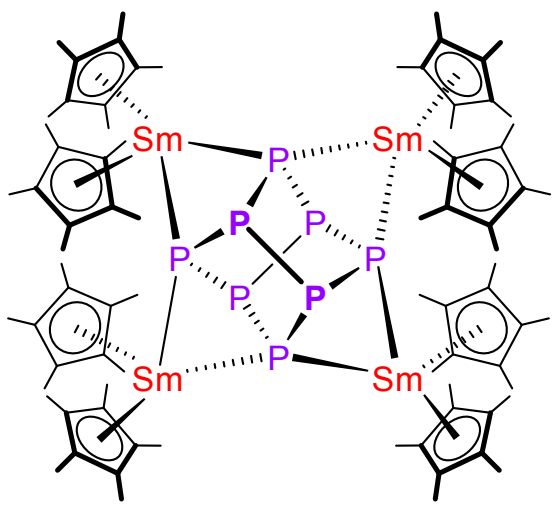

(79)

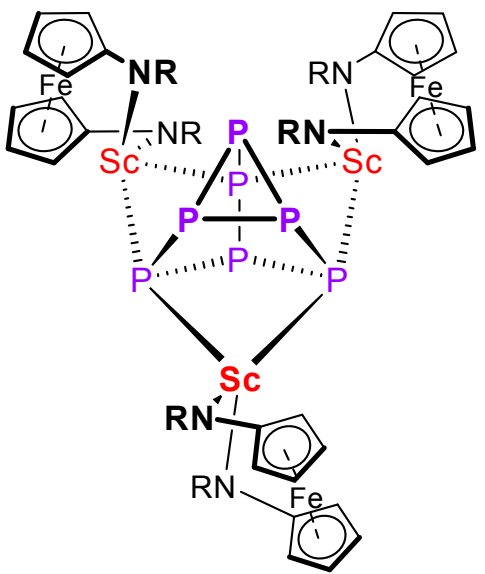

(81)

$\mathrm{R}=\mathrm{Si}^{\mathrm{t} B u M e_{2}}$

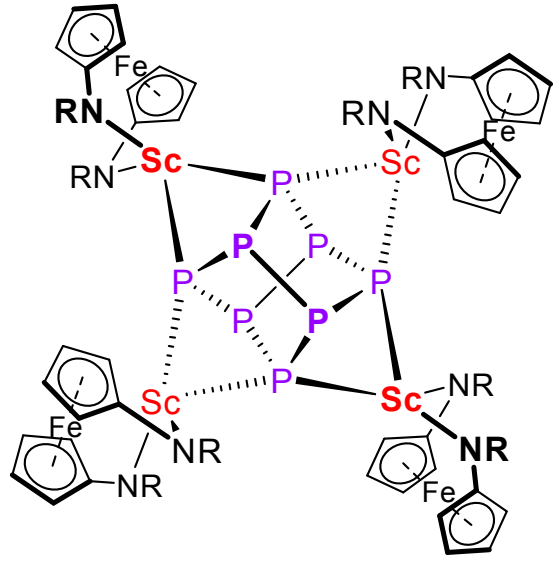

(80)

$\mathrm{R}=\mathrm{Si}^{\mathrm{t}} \mathrm{BuMe}_{2}$

Labelling scheme
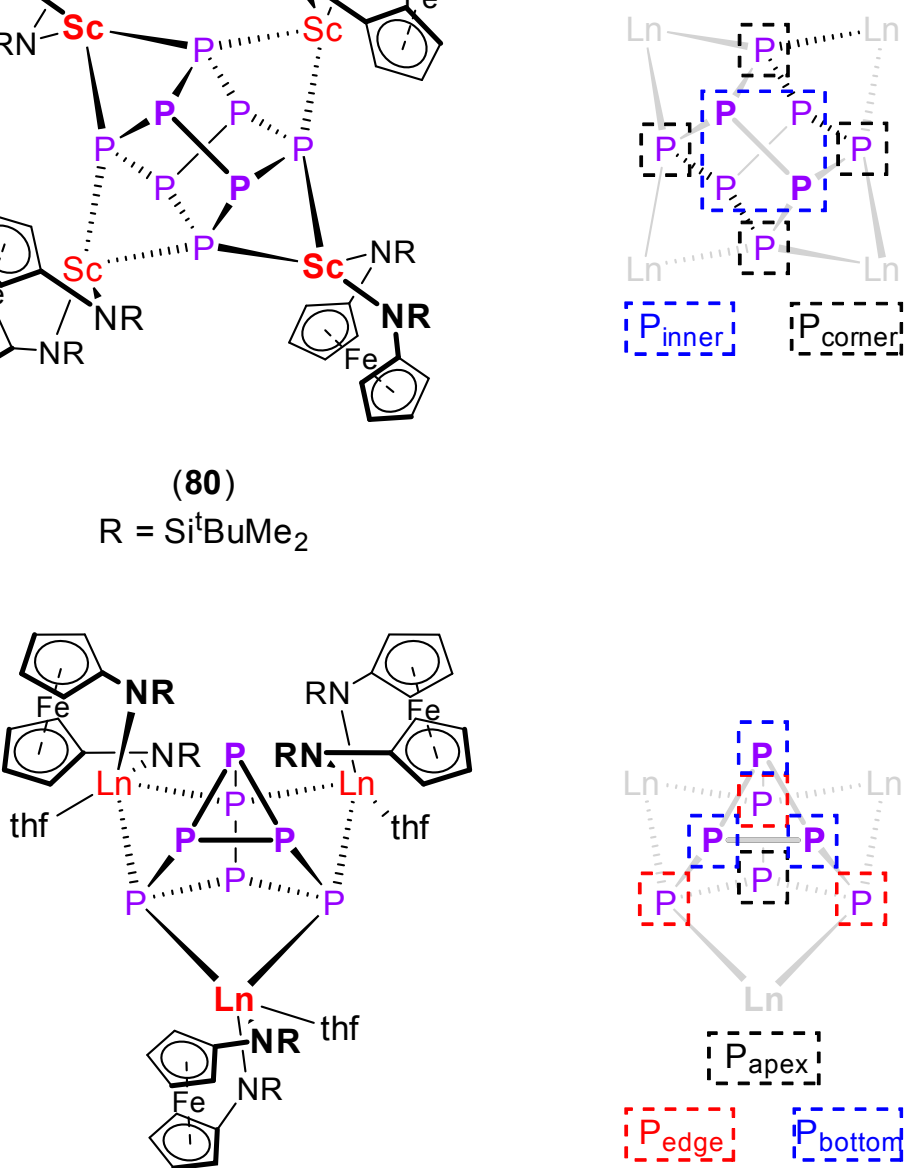

$$
\mathrm{R}=\mathrm{Si}^{\mathrm{t} B u M e_{2}} \quad \mathrm{Ln}=\mathrm{Y}(\mathbf{8 2})
$$

Figure 12. Rare earth complexes resulting from $\mathrm{P}_{4}$ activation.

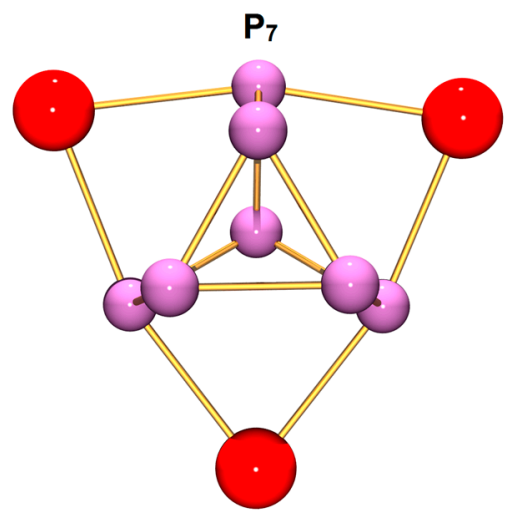

(81), (82)

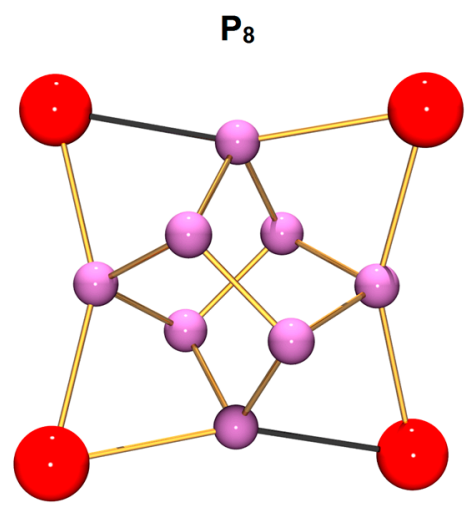

(79), (80)

Figure 13. Overview of the $\operatorname{Ln}_{x} \mathrm{P}_{n}$ structural cores resulting from $\mathrm{P}_{4}$ activation by rare earth complexes. 
Table 5. Summary of rare earth $\mathrm{P}_{4}$ activation complexes.

\begin{tabular}{|c|c|c|c|}
\hline Complex (\#) [Reference] & $\begin{array}{l}\text { Average P-P Bond } \\
\text { Lengths }(\AA)\end{array}$ & $\begin{array}{c}\text { Average } M-P \\
\text { Bond Length } \\
(\AA)\end{array}$ & $\begin{array}{c}{ }^{31} \text { P-NMR } \\
\text { Spectroscopy } \\
\left(298 \text { K) }(\mathrm{ppm}){ }^{\mathrm{a}}\right.\end{array}$ \\
\hline $\mathrm{P}_{4}$ & $2.21[121]$ & - & -488 to $-527[122]$ \\
\hline$\left[\left(\eta^{5}-\mathrm{C}_{5} \mathrm{Me}_{5}\right)_{2} \mathrm{Sm}\right]_{4}\left(\mu_{4}-\eta^{2}: \eta^{2}: \eta^{2}: \eta^{2}-\mathrm{P}_{8}\right)(79)[123]$ & $\begin{array}{l}2.195\left(\mathrm{P}_{\text {corner }}-\mathrm{P}_{\text {inner }}\right) \\
2.291\left(\mathrm{P}_{\text {inner }}-\mathrm{P}_{\text {inner }}\right)\end{array}$ & 3.047 & - \\
\hline $\begin{array}{c}{\left[\left\{\mathrm{Fe}\left(1-\mathrm{NSi}^{\prime} \mathrm{BuMe}_{2}-\mathrm{C}_{5} \mathrm{H}_{4}\right)_{2}\right\} \mathrm{Sc}\right]_{4}\left(\mu_{4}-\eta^{2}: \eta^{2}: \eta^{2}: \eta^{2}-\mathrm{P}_{8}\right)} \\
(\mathbf{8 0})[124]\end{array}$ & $\begin{array}{c}2.204\left(\mathrm{P}_{\text {corner }}-\mathrm{P}_{\text {inner }}\right) \\
2.308\left(\mathrm{P}_{\text {inner }}-\mathrm{P}_{\text {inner }}\right) \\
\end{array}$ & 2.768 & $\begin{array}{r}+45.7 \\
+96.2 \\
\end{array}$ \\
\hline $\begin{array}{c}{\left[\left\{\mathrm{Fe}\left(1-\mathrm{NSi}^{\prime} \mathrm{BuMe}_{2}-\mathrm{C}_{5} \mathrm{H}_{4}\right)_{2}\right\} \mathrm{Sc}\right]_{3}\left(\mu_{3}-\eta^{2}: \eta^{2}: \eta^{2}-\mathrm{P}_{7}\right)(\mathbf{8 1})} \\
{[124]}\end{array}$ & $\begin{array}{c}2.229\left(\mathrm{P}_{\text {bottom }}-\mathrm{P}_{\text {bottom }}\right) \\
2.197\left(\mathrm{P}_{\text {edge }}-\mathrm{P}_{\text {bottom }}\right) \\
2.201\left(\mathrm{P}_{\text {apex }}-\mathrm{P}_{\text {edge }}\right)\end{array}$ & 2.750 & $\begin{array}{c}+23.1 \\
-118.9 \\
-131.4 \\
\end{array}$ \\
\hline $\begin{array}{c}{\left[\left\{\mathrm{Fe}\left(1-\mathrm{NSi}^{t} \mathrm{BuMe}_{2}-\mathrm{C}_{5} \mathrm{H}_{4}\right)_{2}\right\} \mathrm{Y}(\mathrm{thf})\right]_{3}\left(\mu_{3}-\eta^{2}: \eta^{2}: \eta^{2}-\mathrm{P}_{7}\right)} \\
(\mathbf{8 2})[124]\end{array}$ & $\begin{array}{c}2.238\left(\mathrm{P}_{\text {bottom }}-\mathrm{P}_{\text {bottom }}\right) \\
2.176\left(\mathrm{P}_{\text {edge }}-\mathrm{P}_{\text {bottom }}\right) \\
2.188\left(\mathrm{P}_{\text {apex }}-\mathrm{P}_{\text {edge }}\right)\end{array}$ & 2.950 & $\begin{array}{r}-21.1 \\
-82.4 \\
-130.3 \\
\end{array}$ \\
\hline $\begin{array}{c}{\left[\left\{\mathrm{Fe}\left(1-\mathrm{NSi}^{\prime} \mathrm{BuMe}_{2}-\mathrm{C}_{5} \mathrm{H}_{4}\right)_{2}\right\} \mathrm{La}(\mathrm{thf})\right]_{3}\left(\mu_{3}-\eta^{2}: \eta^{2}: \eta^{2}-\mathrm{P}_{7}\right)} \\
(\mathbf{8 3})[125]\end{array}$ & $\begin{array}{c}2.258\left(\mathrm{P}_{\text {bottom }}-\mathrm{P}_{\text {bottom }}\right) \\
2.161\left(\mathrm{P}_{\text {edge }}-\mathrm{P}_{\text {bottom }}\right) \\
2.191\left(\mathrm{P}_{\text {apex }}-\mathrm{P}_{\text {edge }}\right)\end{array}$ & 3.120 & -75 \\
\hline $\begin{array}{c}{\left[\left\{\mathrm{Fe}\left(1-\mathrm{NSi}^{t} \mathrm{BuMe}_{2}-\mathrm{C}_{5} \mathrm{H}_{4}\right)_{2}\right\} \mathrm{Lu}(\mathrm{thf})\right]_{3}\left(\mu_{3}-\eta^{2}: \eta^{2}: \eta^{2}-\mathrm{P}_{7}\right)} \\
(\mathbf{8 4})[125]\end{array}$ & $\begin{array}{c}2.233\left(\mathrm{P}_{\text {bottom }}-\mathrm{P}_{\text {bottom }}\right) \\
2.181\left(\mathrm{P}_{\text {edge }}-\mathrm{P}_{\text {bottom }}\right) \\
2.183\left(\mathrm{P}_{\text {apex }}-\mathrm{P}_{\text {edge }}\right)\end{array}$ & 2.893 & $\begin{array}{c}+0.8 \\
-96.8 \\
-133.3\end{array}$ \\
\hline
\end{tabular}

${ }^{a}$ Referenced to $85 \% \mathrm{H}_{3} \mathrm{PO}_{4}$.

Roesky and co-workers reported the first example of a molecular polyphosphide of the rare earth elements, $\left[\left(\eta^{5}-\mathrm{C}_{5} \mathrm{Me}_{5}\right)_{2} \mathrm{Sm}\right]_{4}\left(\mu 4-\eta^{2}: \eta^{2}: \eta^{2}: \eta^{2}-\mathrm{P}_{8}\right)\left(\right.$ (79) [123]. The samarocene $\left(\eta^{5}-\mathrm{C}_{5} \mathrm{Me}_{5}\right)_{2} \mathrm{Sm}$ activates $\mathrm{P}_{4}$ to yield a $\mathrm{P}_{8}{ }^{4-}$ fragment with a realgar-type structure, a process proposed to be driven by the one-electron oxidation of the divalent samarium metal centre. 79 has molecular $D_{2 d}$ symmetry and the [Cp* $\left.{ }_{2} \mathrm{Sm}\right]$ units bridge the $\mathrm{P}_{8}{ }^{4-}$ cage with $\mathrm{Sm}-\mathrm{P}$ distances in the range of 2.997(2) to 3.100(2) $\AA$. DFT calculations support the strongly ionic character of the Sm-P bonds.

Activation of $\mathrm{P}_{4}$ by group 3 metal centres was first reported by Diaconescu and co-workers [124]. Reaction of the scandium arene inverse-sandwich complexes $\left[\left\{\mathrm{Fe}\left(1-\mathrm{NSi}^{t} \mathrm{BuMe}_{2}-\mathrm{C}_{5} \mathrm{H}_{4}\right)_{2}\right\} \mathrm{Sc}\right]_{2}(\mu$-arene $)$ (arene $=\mathrm{C}_{10} \mathrm{H}_{8}$ or $\mathrm{C}_{14} \mathrm{H}_{10}$ ) with $\mathrm{P}_{4}$ resulted in displacement of the neutral arene and formation of a mixture of the tetrametallic $\left[\left\{\mathrm{Fe}\left(1-\mathrm{NSi}^{t} \mathrm{BuMe}_{2}-\mathrm{C}_{5} \mathrm{H}_{4}\right)_{2}\right\} \mathrm{Sc}\right] 4\left(\mu_{4}-\eta^{2}: \eta^{2}: \eta^{2}: \eta^{2}-\mathrm{P}_{8}\right)$ (80) and trimetallic $\left[\left\{\mathrm{Fe}\left(1-\mathrm{NSi}^{t} \mathrm{BuMe}_{2}-\mathrm{C}_{5} \mathrm{H}_{4}\right)_{2}\right\} \mathrm{Sc}\right]_{3}\left(\mu_{3}-\eta^{2}: \eta^{2}: \eta^{2}-\mathrm{P}_{7}\right)(\mathbf{8 1})$. The mixtures were readily separated and the product distribution could be controlled by the stoichiometry of $\mathrm{P}_{4}$ and the nature of the arene starting material. 80 possesses a realgar-type $\mathrm{P}_{8}{ }^{4-}$ unit whereas 81 contains a Zintl-type $\mathrm{P}_{7}{ }^{3-}$ unit [126], the first example of its formation in the absence of strong alkali metal reducing agents. Solution phase ${ }^{31} \mathrm{P}-\mathrm{NMR}$ spectroscopy demonstrates a diagnostic $\mathrm{AA}^{\prime} \mathrm{A}^{\prime \prime} \mathrm{MM}^{\prime} \mathrm{M}^{\prime \prime} \mathrm{X}$ spin system. The analogous yttrium arene inverse-sandwich complex $\left[\left\{\mathrm{Fe}\left(1-\mathrm{NSi}^{t} \mathrm{BuMe}_{2}-\mathrm{C}_{5} \mathrm{H}_{4}\right)_{2}\right\} \mathrm{Y}(\text { thf })\right]_{2}\left(\mu-\mathrm{C}_{10} \mathrm{H}_{8}\right)$ activates $\mathrm{P}_{4}$ to yield $\left[\left\{\mathrm{Fe}\left(1-\mathrm{NSi}^{t} \mathrm{BuMe}_{2}-\mathrm{C}_{5} \mathrm{H}_{4}\right)_{2}\right\} \mathrm{Y}(\text { thf })\right]_{3}\left(\mu_{3}-\eta^{2}: \eta^{2}: \eta^{2}-\mathrm{P}_{7}\right)(\mathbf{8 2})$ as the sole product where the larger coordination sphere of yttrium is saturated with an additional thf molecule [127]. Importantly, in the context of functionalisation of white phosphorus to organophosphorus compounds, both $\mathbf{8 1}$ and 82 were shown to react with 3 equivalents of $\mathrm{Me} 3 \mathrm{SiI}_{3}$ to yield $\mathrm{P}_{7}\left(\mathrm{SiMe}_{3}\right)_{3}$ and $\left\{\mathrm{Fe}\left(1-\mathrm{NSi}^{t} \mathrm{BuMe}_{2}-\right.\right.$ $\left.\left.\mathrm{C}_{5} \mathrm{H}_{4}\right)_{2}\right\} \mathrm{MI}$ (Scheme 4). 


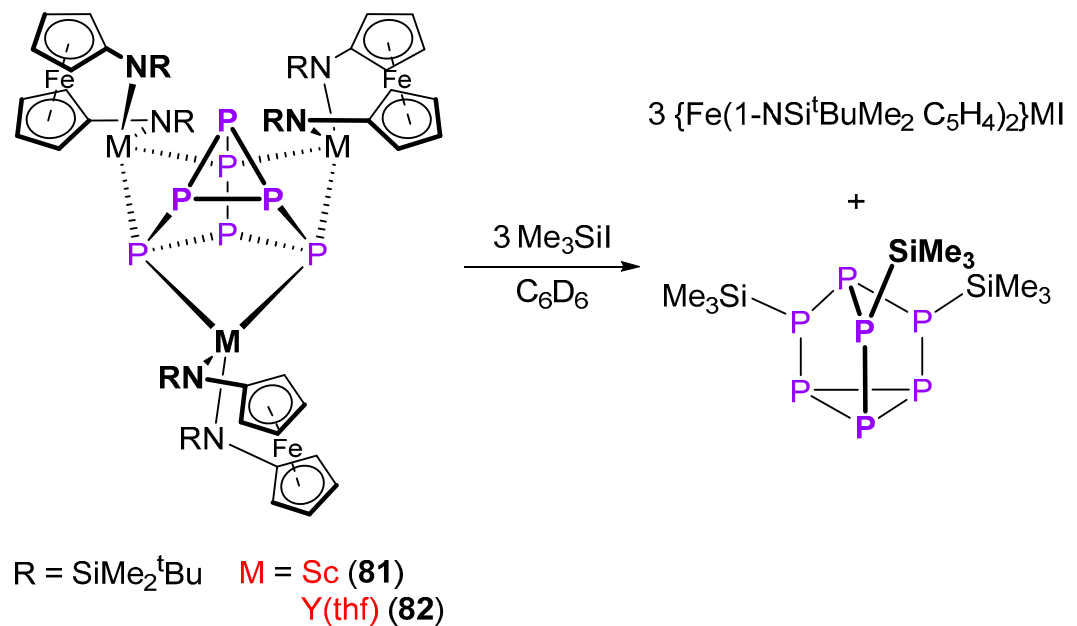

Scheme 4. $\mathrm{P}_{7}^{3-}$ functionalisation with $\mathrm{Me}_{3} \mathrm{SiI}$.

This chemistry was later extended to lanthanum and lutetium using the same methodology, forming $\left[\left\{\mathrm{Fe}\left(1-\mathrm{NSi}^{t} \mathrm{BuMe}_{2}-\mathrm{C}_{5} \mathrm{H}_{4}\right)_{2}\right\} \operatorname{Ln}(\mathrm{thf})\right]_{3}\left(\mu_{3}-\eta^{2}: \eta^{2}: \eta^{2}-\mathrm{P}_{7}\right)(\mathrm{Ln}=\mathrm{La}(\mathbf{8 3}), \mathrm{Lu}(\mathbf{8 4}))$ [125]. The valence tautomerisation of $\mathrm{P}_{7}{ }^{3-}$ in $\mathbf{8 3}$, which occurs at a similar temperature to $\mathrm{Li}_{3} \mathrm{P}_{7}$ but does not require donor solvents, was proposed to take place by a lanthanum-assisted mechanism involving simultaneous formation and breaking of $4 \mathrm{La}-\mathrm{P}$ bonds.

\section{White Phosphorus Activation by Actinide Complexes}

Actinide complexes resulting from $\mathrm{P}_{4}$ activation are illustrated in Figure 14. For reference, average $\mathrm{P}-\mathrm{P}$ and An-P bond lengths obtained from single crystal X-ray diffraction experiments, and ${ }^{31} \mathrm{P}-\mathrm{NMR}$ spectroscopic resonances are summarised in Table 6. The structural cores of complexes $\mathbf{8 5 - 9 1}$ are shown in Figure 15 for clarity.

Table 6. Summary of actinide $\mathrm{P}_{4}$ activation complexes.

\begin{tabular}{|c|c|c|c|}
\hline Complex (\#) [Reference] & $\begin{array}{l}\text { Average P-P Bond } \\
\text { Lengths ( }(\AA)\end{array}$ & $\begin{array}{c}\text { Average An-P } \\
\text { Bond Length } \\
(\AA ̊) \\
\end{array}$ & $\begin{array}{c}{ }^{31} \mathrm{P}-\mathrm{NMR} \\
\text { Spectroscopy } \\
(\mathrm{ppm})^{\mathrm{a}} \\
\end{array}$ \\
\hline $\mathrm{P}_{4}$ & $2.21[121]$ & - & -488 to $-527[122]$ \\
\hline $\begin{array}{c}{\left[\left(\eta^{5}-1,3-{ }^{t} \mathrm{Bu}-\mathrm{C}_{5} \mathrm{H}_{3}\right)_{2} \mathrm{Th}\right]\left(\mu-\eta^{3}: \eta^{3}-c y c l o-\mathrm{P}_{3}\right)\left[\left(\eta^{5}-1,3-{ }^{t} \mathrm{Bu}-\right.\right.} \\
\left.\left.\mathrm{C}_{5} \mathrm{H}_{3}\right)_{2} \mathrm{ThCl}\right](\mathbf{8 5})[128]\end{array}$ & 2.185 & 2.913 & $-75.7(293 \mathrm{~K})$ \\
\hline$\left[\left(\eta^{5}-1,3-{ }^{t} \mathrm{Bu}-\mathrm{C}_{5} \mathrm{H}_{3}\right)_{2} \mathrm{Th}\right]_{2}\left(\mu-\eta^{3}: \eta^{3}-\mathrm{P}_{6}\right)(\mathbf{8 6})[128]$ & 2.234 & $\begin{array}{l}2.904\left(\text { Th- } \eta^{2}-\mathrm{P}\right) \\
2.844\left(\text { Th- }-\eta^{1}-\mathrm{P}\right)\end{array}$ & $\begin{array}{l}+125.4(293 \mathrm{~K}) \\
-41.9(293 \mathrm{~K})\end{array}$ \\
\hline$\left[\left\{\left(3,5-\mathrm{Me}-\mathrm{C}_{6} \mathrm{H}_{3}\right)\left({ }^{t} \mathrm{Bu}\right) \mathrm{N}\right\}_{3} \mathrm{U}\right]\left(\mu-\eta^{4}: \eta^{4}-\right.$ cyclo- $\left.\mathrm{P}_{4}\right)(87)[129]$ & 2.160 & 3.127 & +794 \\
\hline$\left[\left\{\left(3,5-\mathrm{Me}-\mathrm{C}_{6} \mathrm{H}_{3}\right)(\mathrm{Ad}) \mathrm{N}\right\}_{3} \mathrm{U}\right]\left(\mu-\eta^{4}: \eta^{4}-c y c l o-\mathrm{P}_{4}\right)(\mathbf{8 8})[129]$ & 2.159 & 3.124 & +803 \\
\hline $\begin{array}{c}{\left[\left(\eta^{5}-\mathrm{C}_{5} \mathrm{Me}_{5}\right)\left(\eta^{8}-1,4-\mathrm{Si}^{i} \mathrm{Pr}_{3}-\mathrm{C}_{8} \mathrm{H}_{6}\right) \mathrm{U}\right]_{2}\left(\mu-\eta^{2}: \eta^{2}-c y c l o-\mathrm{P}_{4}\right)(\mathbf{8 9})} \\
{[130]}\end{array}$ & 2.150 & 2.977 & +718 \\
\hline$\left[\mathrm{HC}\left(\mathrm{SiMe}_{2} \mathrm{~N}-4-\mathrm{Me}-\mathrm{C}_{6} \mathrm{H}_{4}\right)_{3} \mathrm{U}\right]_{3}\left(\mu_{3}-\eta^{2}: \eta^{2}: \eta^{2}-\mathrm{P}_{7}\right)(\mathbf{9 0})[130]$ & $\begin{array}{c}2.249\left(\mathrm{P}_{\text {bottom}}-\mathrm{P}_{\text {bottom }}\right) \\
2.187\left(\mathrm{P}_{\text {edge }}-\mathrm{P}_{\text {bottom }}\right) \\
2.209\left(\mathrm{P}_{\text {apex }}-\mathrm{P}_{\text {edge }}\right) \\
\end{array}$ & 2.990 & - \\
\hline$\left[\left\{\mathrm{N}\left(\mathrm{CH}_{2} \mathrm{CH}_{2} \mathrm{NSi}^{i} \mathrm{Pr}_{3}\right)_{3}\right\} \mathrm{U}_{2}\left(\mu-\eta^{5}: \eta^{5}-\right.\right.$ cyclo- $\left.\mathrm{P}_{5}\right)(\mathbf{9 1})[131]$ & 2.006 & 3.280 & - \\
\hline
\end{tabular}

${ }^{\text {a }}$ Referenced to $85 \% \mathrm{H}_{3} \mathrm{PO}_{4}$. 


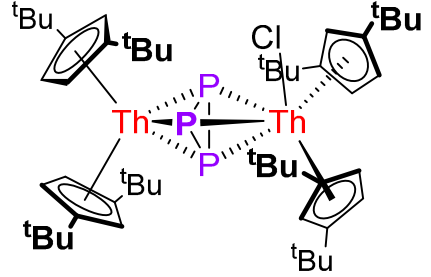

(85)

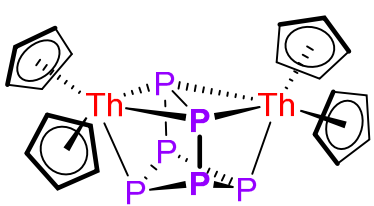

(86)

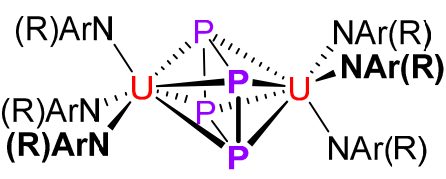

$\mathrm{Ar}=3,5-\mathrm{Me}-\mathrm{C}_{6} \mathrm{H}_{3} \quad \mathrm{R}={ }^{\mathrm{t}} \mathrm{Bu}(87)$ Ad (88)

1,3- ${ }^{\mathrm{t}} \mathrm{Bu}$ groups on $\mathrm{Cp}$ rings omitted for clarity

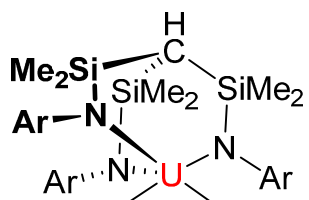

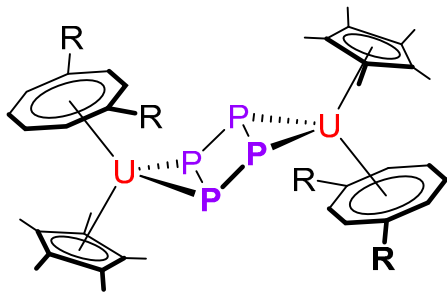

(89)

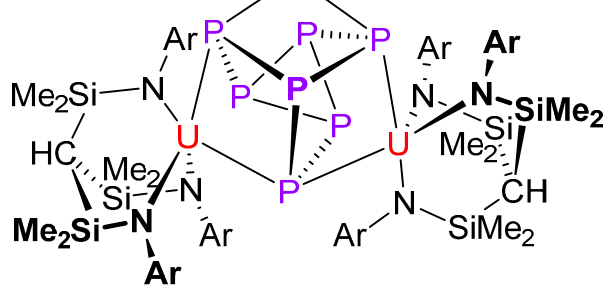

(90)

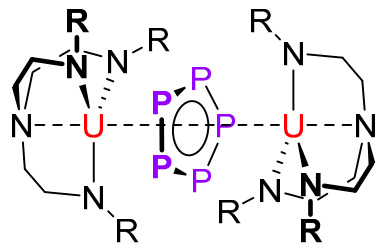

(91)

Figure 14. Actinide complexes resulting from $\mathrm{P}_{4}$ activation.

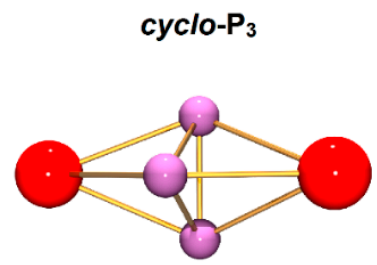

(85)

cyclo- $\mathrm{P}_{5}$

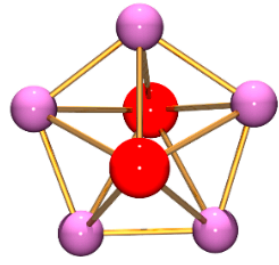

(91)

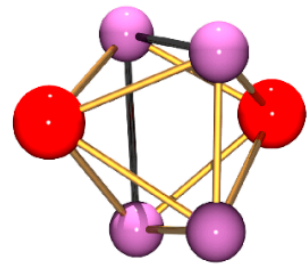

(87), (88)

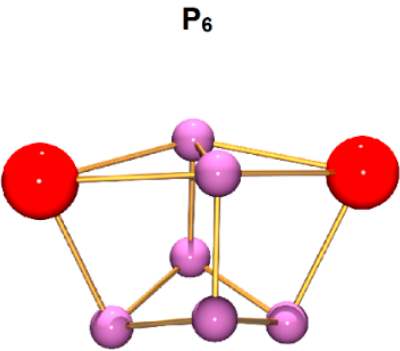

(86)

cyclo- $\mathrm{P}_{4}$

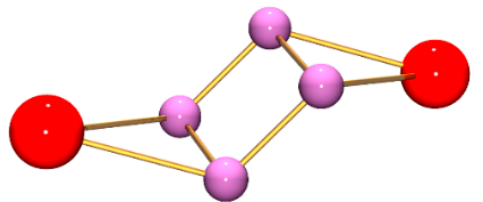

(89)

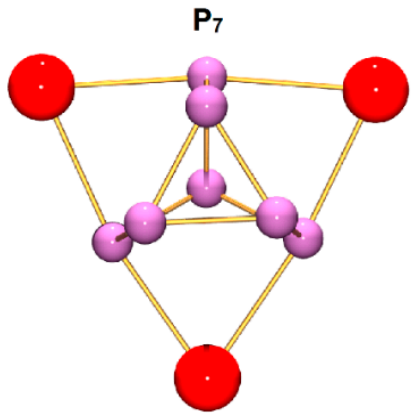

(90)

Figure 15. Overview of the $\mathrm{An}_{x} \mathrm{P}_{n}$ structural cores resulting from $\mathrm{P}_{4}$ activation by actinide complexes.

The first report of $\mathrm{P}_{4}$ activation by an actinide complex came from Scherer et al., using thorium [128]. The butadiene complex $\left(\eta^{5}-1,3-{ }^{t} \mathrm{Bu}-\mathrm{C}_{5} \mathrm{H}_{3}\right)_{2} \mathrm{Th}\left(\eta^{4}-\mathrm{C}_{4} \mathrm{H}_{6}\right)$ reacts with $\mathrm{P}_{4}$ at $100{ }^{\circ} \mathrm{C}$ in the presence of 
$\mathrm{MgCl}_{2}\left(\mathrm{OEt}_{2}\right)$ to yield $\left[\left(\eta^{5}-1,3-{ }^{t} \mathrm{Bu}-\mathrm{C}_{5} \mathrm{H}_{3}\right)_{2} \mathrm{Th}\right]\left(\mu-\eta^{3}: \eta^{3}-\right.$ cyclo $\left.-\mathrm{P}_{3}\right)\left[\left(\eta^{5}-1,3-{ }^{t} \mathrm{Bu}-\mathrm{C}_{5} \mathrm{H}_{3}\right)_{2} \mathrm{ThCl}\right](\mathbf{8 5})$. In the absence of $\mathrm{MgCl}_{2}\left(\mathrm{OEt}_{2}\right),\left[\left(\eta^{5}-1,3-{ }^{t} \mathrm{Bu}-\mathrm{C}_{5} \mathrm{H}_{3}\right)_{2} \mathrm{Th}\right]_{2}\left(\mu-\eta^{3}: \eta^{3}-\mathrm{P}_{6}\right)(\mathbf{8 6})$ was afforded as a consequence of $\mathrm{P}_{4}$ fragmentation and subsequent catenation. 85 features a cyclo- $\mathrm{P}_{3}{ }^{3-}$ unit and formal $\mathrm{Th}^{\mathrm{IV}}$ centres. In the solid state structure, the coordination environment about the thorium centres is trigonal planar and tetrahedral ( $\mathrm{Cl}$ bound) with Th-P distances ranging from 2.809(6) to 2.974(8) $\AA$. The P-P distances are 2.171(9), 2.192(9) and 2.192(8) $\AA$. Only a single broad resonance is observed in the ${ }^{31} \mathrm{P}-\mathrm{NMR}$ spectrum at $293 \mathrm{~K}$ but cooling to $193 \mathrm{~K}$ leads to splitting and the observation of an $\mathrm{A}_{2} \mathrm{~B}$ system; the barrier to rotation of the cyclo- $\mathrm{P}_{3}$ unit was estimated to be $c a .44 \mathrm{~kJ} \cdot \mathrm{mol}^{-1} .86$ contains a $\mathrm{P}_{6}{ }^{4-}$ bicycle with thorium metal centres capping the five-membered rings. Th- $\mathrm{P}$ bond lengths in the solid state structure range from 2.840(7) to 2.919(7) $\AA$.

The following report of $\mathrm{P}_{4}$ activation came over a decade later and was the first using a uranium complex [129]. The $\mathrm{U}^{\mathrm{III}}$ tris(amide) $\mathrm{U}\{\mathrm{N}(\mathrm{R}) \mathrm{Ar}\}_{3}(\mathrm{thf})\left(\mathrm{R}={ }^{t} \mathrm{Bu}\right.$ or $\left.\mathrm{Ad}, \mathrm{Ar}=3,5-\mathrm{Me}-\mathrm{C}_{6} \mathrm{H}_{3}\right)$ reacts with 0.5 equivalents of $\mathrm{P}_{4}$ to yield $\left[\{\operatorname{Ar}(\mathrm{R}) \mathrm{N}\}{ }_{3} \mathrm{U}\right]\left(\mu-\eta^{4}: \eta^{4}-c y c l o-\mathrm{P}_{4}\right)\left(\mathrm{R}={ }^{t} \mathrm{Bu}(\mathbf{8 7}), \mathrm{Ad}(\mathbf{8 8})\right)$ which contains a cyclo- $\mathrm{P}_{4}{ }^{2-}$ unit and where the metal centres have been formally oxidised to $\mathrm{U}^{\mathrm{IV}}$. In the solid state structures, the average P-P bond distance is $2.159 \AA$ and the $\mathrm{P}-\mathrm{P}-\mathrm{P}$ angle is $90^{\circ}$; both statistically equivalent across the two structures. Resonances at 794 and 803 ppm were observed for 87 and 88 respectively in the ${ }^{31} \mathrm{P}-\mathrm{NMR}$ spectrum. Computational studies implied that the U-P bonding character is largely ionic with the presence of a weak $\delta$-bonding interaction between filled $U$ df hybrid orbitals and the $\mathrm{P}_{4}{ }^{2-}$ LUMO.

Cloke and co-workers described the related cyclo- $\mathrm{P}_{4}$ example $\left[\left(\eta^{5}-\mathrm{C}_{5} \mathrm{Me}_{5}\right)\left(\eta^{8}-1,4-\mathrm{Si}^{i} \mathrm{Pr}_{3}-\mathrm{C}_{8} \mathrm{H}_{6}\right) \mathrm{U}_{2}\right.$ $\left(\mu-\eta^{2}: \eta^{2}-\right.$ cyclo- $\left.\mathrm{P}_{4}\right)$ (89) which was prepared from $\left(\eta^{5}-\mathrm{C}_{5} \mathrm{Me}_{5}\right)\left(\eta^{8}-1,4-\mathrm{Si}^{i} \mathrm{Pr}_{3}-\mathrm{C}_{8} \mathrm{H}_{6}\right) \mathrm{U}($ thf $)$ and 0.5 equivalents of $\mathrm{P}_{4}$ [130]. This was the first example of the $\mu-\eta^{2}: \eta^{2}-\mathrm{P}_{4}$ coordination mode [132,133] and DFT studies on a model system $\left[\left(\eta^{5}-\mathrm{C}_{5} \mathrm{H}_{5}\right)\left(\eta^{8}-\mathrm{C}_{8} \mathrm{H}_{8}\right) \mathrm{U}\right]\left(\mu-\eta^{2}: \eta^{2}-c y c l o-\mathrm{P}_{4}\right)$ support the formulation of the dimer with $\mathrm{P}_{4}{ }^{2-}$ and $\mathrm{U}^{\mathrm{IV}}$ oxidation states. The tilted cyclo- $\mathrm{P}_{4}{ }^{2-}$ unit leads to $\mathrm{U}-\mathrm{P}$ bonding interactions involving both $\sigma$ and $\pi$ orbitals. The wedge shaped nature of the sterically demanding $\left(\eta^{5}-\mathrm{C}_{5} \mathrm{Me}_{5}\right)\left(\eta^{8}-1,4-\mathrm{Si}^{i} \mathrm{Pr}_{3}-\mathrm{C}_{8} \mathrm{H}_{6}\right) \mathrm{U}$ fragment likely results in the slipped $\mu-\eta^{2}: \eta^{2}$ coordination mode.

Following on from the rare earth inverse sandwich complexes that resulted in $\mathrm{P}_{8}{ }^{4-}$ and $\mathrm{P}_{7}{ }^{3-}$ clusters, and $\mathrm{P}_{7}^{3-}$ functionalisation [124,125], Liddle and co-workers reported the reaction of $\left[\mathrm{HC}\left(\mathrm{SiMe}_{2} \mathrm{NAr}\right)_{3} \mathrm{U}_{2}\left(\mu-\eta^{6}-\eta^{6}-\mathrm{C}_{6} \mathrm{H}_{5} \mathrm{CH}_{3}\right)\left(\mathrm{Ar}=4-\mathrm{Me}-\mathrm{C}_{6} \mathrm{H}_{4}\right)\right.$ with 1.1 equivalents of $\mathrm{P}_{4}$ which afforded the first actinide Zintl complex [HC( $\left.\operatorname{SiMe}_{2} \mathrm{NAr}\right)_{3} \mathrm{U}_{3}\left(\mu_{3}-\eta^{2}: \eta^{2}: \eta^{2}-\mathrm{P}_{7}\right)(\mathbf{9 0})$ [134]. U-P bonding was determined to be essentially ionic. Interestingly, reaction of $\mathbf{9 0}$ with a number of electrophiles under ambient conditions led to functionalisation of the $\mathrm{P}_{7}{ }^{3-}$ unit and liberation of $\mathrm{P}_{7} \mathrm{R}_{3}\left(\mathrm{R}=\mathrm{SiMe}_{3}, \mathrm{Me}, \mathrm{Ph}\right.$, $\mathrm{Li}($ tmeda)) after $\mathrm{P}-\mathrm{Si}, \mathrm{P}-\mathrm{C}$ or $\mathrm{P}-\mathrm{Li}$ bond formation. Though not catalytic, 90 could be regenerated from this reaction mixture and two turnovers achieved demonstrating a significant step towards controlled $\mathrm{P}_{4}$ activation under mild conditions.

Very recently, Liddle and co-workers described the first example of a cyclo- $\mathrm{P}_{5}$ complex resulting from activation of $\mathrm{P}_{4}$ by an f-block complex [131]. $\left[\left\{\mathrm{N}\left(\mathrm{CH}_{2} \mathrm{CH}_{2} \mathrm{NSi}^{i} \mathrm{Pr}_{3}\right)_{3}\right\} \mathrm{U}_{2}\left(\mu-\eta^{5}: \eta^{5}-c y c l o-\mathrm{P}_{5}\right)(\mathbf{9 1})\right.$ was prepared by reaction of $\left\{\mathrm{N}\left(\mathrm{CH}_{2} \mathrm{CH}_{2} \mathrm{NSi}^{i} \mathrm{Pr}_{3}\right)_{3}\right\} \mathrm{U}$ with 0.25 equivalents of $\mathrm{P}_{4}$. Spectroscopic and magnetic measurements support oxidation to afford $\mathrm{U}^{\mathrm{IV}}$ centres and charge transfer resulting in a formal $\mathrm{P}_{5}{ }^{2-}$ ligand in this inverse sandwich complex. Despite the isolobal analogy of cyclo- $\mathrm{P}_{5}$ with the cyclopentadienyl anion, which bonds to metal centres using primarily $\sigma$ - and $\pi$-bonding, calculations 
on 91 suggest that the principal U-P interactions involve polarised $\delta$-bonding and this can be attributed to the energetically available uranium $5 f$ orbitals of correct $\delta$-symmetry.

Compared to both rare earth and actinide complexes, the activation of $\mathrm{P}_{4}$ by transition metal complexes have proven to result in a wide variety of activation products [58,59]; with notable examples including fragmentation resulting in terminal and bridging $\mathrm{P}_{1}$ ligands [135-137], $\mathrm{P}_{2}$ ligands [138], cyclo- $\mathrm{P}_{3}$ ligands [56,139], fragmentation to other $\mathrm{P}_{4}$ ligands [140], coordination of $\mathrm{P}_{4}$ tetrahedra [141,142], and expansion to $\mathrm{P}_{n}(n=5-14)$ ligands [143-146]. More significantly, functionalisation of these phosphorus units has also been observed.

\section{Arsenic, Antimony and Bismuth Activation by Rare Earth and Actinide Complexes}

There is only a single example of molecular arsenic activation by a rare earth or actinide metal complex. Reaction of the thorium butadiene complex $\left(\eta^{5}-1,3-{ }^{t} \mathrm{Bu}-\mathrm{C}_{5} \mathrm{H}_{3}\right)_{2} \mathrm{Th}\left(\eta^{4}-\mathrm{C}_{4} \mathrm{H}_{6}\right)$ with As4 (yellow arsenic) in boiling xylene affords $\left[\left(\eta^{5}-1,3-{ }^{t} \mathrm{Bu}-\mathrm{C}_{5} \mathrm{H}_{3}\right)_{2} \mathrm{Th}\right]_{2}\left(\mu-\eta^{3}: \eta^{3}-\mathrm{As} 6\right)$ in analogy to the previously reported $\mathrm{P}_{4}$ chemistry of Scherer et al. (Figure 16) [147]. In the solid state molecular structure; the average Th- $\left(\eta^{2}-A_{6}\right)$ bonds are $3.027 \AA$, average Th- $\left(\eta^{1}-\mathrm{As}\right)$ bonds are $2.922 \AA$ and average As-As bonds are $2.459 \AA$.

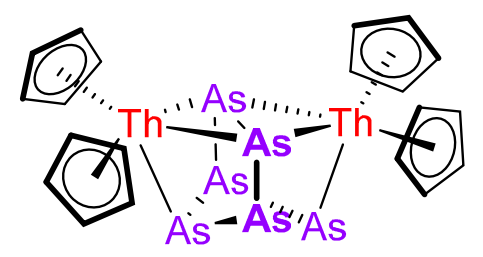

(92)

1,3-'Bu groups on $\mathrm{Cp}$

rings omitted for clarity

Figure 16. Actinide complexes resulting from As4 activation.

In contrast, activation of molecular arsenic by homogeneous transition metal complexes is considerably more diverse; formation of cyclo-As $n$ ligands $(n=3-6,8)$ [54,148], metal arsenic clusters [149], coordination of intact As4 tetrahedra to metal ions [133,150,151], fragmentation into As2 and other As4 ligands [152-154], catenation to $\mathrm{As}_{10}$ and $\mathrm{As}_{12}$ ligands [155], reactions to form $\mathrm{P}_{n} \mathrm{As}_{m}$ ligands [156], and full As4 fragmentation resulting in terminal $\mathrm{M} \equiv \mathrm{As}$ arsenide bonds [157] have all been reported.

Beyond arsenic in group 15 are antimony and bismuth. While activation of molecular forms of these elements is unlikely, it is worth noting that Scheer and co-workers reported a tungsten terminal stibido

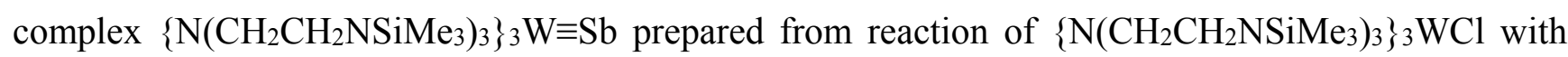
$\mathrm{LiSb}(\mathrm{H})\left\{\mathrm{CH}\left(\mathrm{SiMe}_{3}\right)_{2}\right\}$ [158], while Breunig et al., have reported $\left[\left(\eta^{5}-\mathrm{C}_{5} \mathrm{Me}\right)_{2} \mathrm{Mo}(\mathrm{CO})_{2}\right]\left(\right.$ cyclo-Sb$\left._{3}\right)$ and $\left[\left(\eta^{5}-\mathrm{C}_{5} \mathrm{H}_{5}\right)_{2} \mathrm{Mo}(\mathrm{CO})_{2}\right]\left(\right.$ cyclo-Sb3), which are a result of reaction of $\left[\left(\eta^{5}-\mathrm{Cp}^{\mathrm{R}}\right)_{2} \mathrm{Mo}(\mathrm{CO})_{3}\right]_{2}$ with $\left.{ }^{t} \mathrm{BuSb}\right)_{4}$ [2]. In terms of rare earth complexes; [( $\left.\left.\eta^{5}-\mathrm{C}_{5} \mathrm{Me}_{5}\right)_{2} \mathrm{Sm}\right]_{2}\left(\mu_{3}-\eta^{1}: \eta^{2}: \eta^{2}-\mathrm{Sb}_{3}\right)\left\{\left(\eta^{5}-\right.\right.$ $\left.\left.\mathrm{C}_{5} \mathrm{Me}_{5}\right)_{2}(\mathrm{thf}) \mathrm{Sm}\right\}$ and $\left[\left(\eta^{5}-\mathrm{C}_{5} \mathrm{Me}_{5}\right)_{2} \mathrm{Sm}\right]_{2}\left(\eta^{2}: \eta^{2}-\mathrm{Bi}_{2}\right)$ were prepared by reaction of $\left[\left(\eta^{5}-\mathrm{C}_{5} \mathrm{Me}_{5}\right)_{2} \mathrm{Sm}\right]_{2}$ with $\mathrm{SbPh}_{3}$ and $\mathrm{BiPh}_{3}$ respectively $[3,4]$. 


\section{Conclusions and Perspectives}

To date, a wide range of rare earth dinitrogen complexes have been prepared (1-68), including group 3 metal ions and $4 \mathrm{f}$ elements at both ends of the periodic table, despite the limited radial extension of the $4 \mathrm{f}$ orbitals and the trivalent oxidation state being the most prevalent. In fact, apart from the very first f-element dinitrogen complex (4), all of the other complexes are air-sensitive but stable to $\mathrm{N}_{2}$ dissociation in vacuo. Reduction of $\mathrm{N}_{2}$ to $\mathrm{N}_{2}{ }^{2-}$ has most commonly been achieved with the $\left[\mathrm{A}_{2}(\mathrm{thf})_{x} \mathrm{Ln}\right]_{2}\left(\mu-\eta^{2}: \eta^{2}-\mathrm{N}_{2}\right)$ structural motif (1-38) whereas examples of reduction to $\mathrm{N}_{2}{ }^{4-}$ have all involved more complex multidentate ligands (61-68). The nature of the bonding in complexes of the form $\left[\mathrm{A}_{2}(\mathrm{thf})_{x} \mathrm{Ln}\right]_{2}\left(\mu-\eta^{2}: \eta^{2}-\mathrm{N}_{2}\right)$ has been extensively studied for both group 3, and closed and open shell $4 \mathrm{f}^{n}$ metal ions demonstrating that the $\mathrm{Ln}-\mathrm{N}\left(\mathrm{N}_{2}\right)$ bonding is based on a $\mathrm{Ln}$ nd- $\mathrm{N}_{2} \pi^{*}$ interaction. These systems have allowed for the first definitive characterisation of the $\mathrm{N}_{2}{ }^{3-}$ radical reduction product of dinitrogen $(\mathbf{4 4}, \mathbf{4 7})$ and this has now been extended to many of the rare earth elements $(\mathbf{4 5}$, 46, 48-60). Isolation of the $\mathrm{N}_{2}{ }^{3-}$ radical in homogeneous complexes is significant since it is likely to be a transient species in other transition metal systems and may also have a role in biological $\mathrm{N}_{2}$ fixation.

In terms of reactivity, the rare earth $\mathrm{N}_{2}$ complexes prepared thus far react with $\mathrm{N}_{2}$ dissociation rather than $\mathrm{N}_{2}$ cleavage and functionalisation [77,159-161]. Related to this, the understanding of the nature of bonding of $\mathrm{Ln}-\mathrm{N}$ multiple bonds is of fundamental interest and the isolation of terminal imido $(\mathrm{Ln}=\mathrm{NR})$ complexes has only recently been reported [162-164]. It is also significant to consider the reactivity of $\mathrm{Ln}-\mathrm{N}_{2}$ complexes in the context of other low electron count early transition metal complexes; here, $\mathrm{N}_{2}$ cleavage or functionalisation can be achieved through ligand induced reductive cleavage [165-173], and more generally, $\mathrm{N}-\mathrm{N}$ bond scission of reduced $\mathrm{N}_{2}$ derivatives can be attained through metal-ligand cooperativity [174,175].

Actinide dinitrogen complexes are much rarer, with just 8 examples of well-defined molecular uranium complexes (69-76). Of these, only the heterobimetallic U-Mo end-on $\mathrm{N}_{2}$ complexes (70, 71) and recently prepared $\mathrm{U}^{\mathrm{IV}}$ aryloxide and siloxide side-on $\mathrm{N}_{2}$ complexes $(\mathbf{7 5}, \mathbf{7 6})$ are thermally robust and stable when exposed to vacuum. Key steps forward have been made in the understanding of U-N $\left(\mathrm{N}_{2}\right)$ bonding in the side-on $\mathrm{N}_{2}$ complexes as a polar covalent $\mathrm{U} 5 \mathrm{f}-\mathrm{N}_{2} \pi^{*}$ interaction, and in the rationalisation of the differences between solid state $\mathrm{N}-\mathrm{N}$ bond lengths and the overall electronic structure of these complexes. This understanding, in combination with well-designed ligand sets may lead the way in the preparation of other isolable actinide dinitrogen complexes for further study.

Though the isolated actinide $\mathrm{N}_{2}$ complexes, like the rare earth $\mathrm{N}_{2}$ complexes, tend to react with $\mathrm{N}_{2}$ loss rather than $\mathrm{N}_{2}$ functionalisation or cleavage, there are two reports of such reactivity. Importantly, in both cases, the putative $\mathrm{An}\left(\mathrm{N}_{2}\right)$ complex was not observed. Cleavage of $\mathrm{N}_{2}$ by a uranium tetra-calix-pyrrole complex results in a bimetallic complex with bridging nitrides (78) whereas a thorium bisphenolate complex activates and functionalises $\mathrm{N}_{2}$ to a parent amide ligand $\left[\mathrm{NH}_{2}\right]^{-}($(79) through an unknown mechanism. Both reactions occur in the presence of an external reductant. Examples of isolable terminal uranium nitrides $(\mathrm{U} \equiv \mathrm{N})$, derived from $\mathrm{NaN}_{3}$, have only recently been reported [176-178], but it has already been demonstrated that these systems are capable of nitride functionalisation. 78 and 79 remain standout examples in demonstrating that actinide complexes can both cleave and functionalise $\mathrm{N}_{2}$, but also highlight how much more remains to be understood in this field. 
$\mathrm{P}_{4}$ activation by rare earth complexes has led to both $\mathrm{P}_{8}{ }^{4-}$ ions with realgar-type structures $(\mathbf{7 9}, \mathbf{8 0})$, and $\mathrm{P}_{7}{ }^{3-}$ ions (81-84) using cyclopentadienyl and amido ancillary ligands. Promisingly, functionalisation of the $\mathrm{P}_{7}{ }^{3-}$ unit in $\mathbf{8 1}$ and $\mathbf{8 2}$ was found possible using $\mathrm{Me}_{3} \mathrm{SiI}$ to afford $\mathrm{P}_{7}\left(\mathrm{SiMe}_{3}\right)_{3}$. This is an interesting prospect for the synthesis of organophosphorus compounds from a $\mathrm{P}_{4}$ building block. Actinide $\mathrm{P}_{4}$ activation results in a more diverse array of phosphorus ligands; $\mathrm{P}_{3}{ }^{3-}(\mathbf{8 5}), \mathrm{P}_{6}{ }^{4-}$, (86), cyclo- $\mathrm{P}_{4}{ }^{2-}(\mathbf{8 7 - 8 9}), \mathrm{P}_{7}{ }^{3-}(\mathbf{9 0})$, and cyclo- $\mathrm{P}_{5}{ }^{2-}(\mathbf{9 1})$. Similar to rare earth chemistry, the $\mathrm{P}_{7}{ }^{3-}$ ions in 90 could be functionalised by $\mathrm{P}-\mathrm{Si}, \mathrm{P}-\mathrm{C}$ or $\mathrm{P}-\mathrm{Li}$ bond formation to afford $\mathrm{P}_{7} \mathrm{R}_{3}$ units and this reaction cycle could be completed with two turnovers. 91 is the first example of an f-element being able to fragment and catenate $\mathrm{P}_{4}$ to cyclo- $\mathrm{P}_{5}{ }^{2-}$. Despite the parallels with the cyclopentadienyl ligand, calculations suggest the U-P (cyclo- $\left.\mathrm{P}_{5}\right)$ interaction to be based on polarised $\delta$-bonding and electronic structure in these systems can be described as $\left[\mathrm{U}^{\mathrm{IV}}\right]_{2}\left(\mathrm{P}_{5}{ }^{2-}\right)$. Putting this area into perspective, transition metals have already been shown to activate molecular phosphorus and, in limited examples, to result in further functionalisation.

There remains only a lone example of arsenic activation by a thorium butadiene complex leading to a $\mathrm{P}_{6}$ cage (92) and no examples using rare earth metals. The first examples of crystallographically characterised uranium arsenide $\left(\mathrm{U}-\mathrm{AsH}_{2}\right)$, arsenidene $(\mathrm{U}=\mathrm{AsH})$ and arsenido $(\mathrm{U} \equiv \mathrm{AsK})$ complexes have only recently been reported, using $\mathrm{KAsH}_{2}$ as a source of arsenic [179]. These compounds raise the question of the diverse reactivity that actinide complexes could be expected to show with molecular arsenic and whether the formation of an unsupported, terminal actinide arsenide bond $(\mathrm{M} \equiv \mathrm{As})$ is accessible.

It is clear that molecular pnictogen activation by rare earth and actinide metal complexes is an exciting field of study which remains underdeveloped with respect to transition metals and main group elements. These unique metals offer the potential of new reactivity and functionalisation chemistry with the pnictogen elements, while the fundamental study of M-pnictogen bonds remains important.

\section{Acknowledgments}

Zoë R. Turner thanks Prof. Dermot O’Hare (University of Oxford), SCG Chemicals for financial support and a SCG Research Fellowship, and Trinity College for a Junior Research Fellowship.

\section{Conflicts of Interest}

The author declares no conflict of interest.

\section{References}

1. Kaltsoyannis, N. Does covalency increase or decrease across the actinide series? Implications for minor actinide partitioning. Inorg. Chem. 2013, 52, 3407-3413.

2. Breunig, H.J.; Rösler, R.; Lork, E. Complexes with $\mathrm{Sb}_{2}$ and cyclo-Sb3 ligands: The tetrahedranes $\left[\left\{\mathrm{C}_{5} \mathrm{H}_{5}(\mathrm{CO})_{2} \mathrm{Mo}_{2} \mathrm{Sb}_{2}\right],\left[\mathrm{C}_{5} \mathrm{H}_{5}(\mathrm{CO})_{2} \mathrm{MoSb}_{3}\right]\right.$, and $\left[\mathrm{C}_{5} \mathrm{Me}_{5}(\mathrm{CO})_{2} \mathrm{MoSb}_{3}\right]$. Angew. Chem. Int. Ed. 1997, 36, 2819-2821. 
3. Evans, W.J.; Gonzales, S.L.; Ziller, J.W. The utility of $\left(\mathrm{C}_{5} \mathrm{Me}_{5}\right)_{2} \mathrm{Sm}$ in isolating crystallographically characterizable zintl ions. X-ray crystal structure of a samarium complex of $\left(\mathrm{Sb}_{3}\right)^{3-}$. J. Chem. Soc. Chem. Commun. 1992, 1138-1139.

4. Evans, W.J.; Gonzales, S.L.; Ziller, J.W. Organosamarium-mediated synthesis of bismuthbismuth bonds: X-ray crystal structure of the first dibismuth complex containing a planar $\mathrm{M}_{2}(\mu-$ $\left.\eta^{2}: \eta^{2}-\mathrm{Bi}_{2}\right)$ unit. J. Am. Chem. Soc. 1991, 113, 9880-9882.

5. Hoffman, B.M.; Dean, D.R.; Seefeldt, L.C. Climbing nitrogenase: Toward a mechanism of enzymatic nitrogen fixation. Acc. Chem. Res. 2009, 42, 609-619.

6. $\mathrm{Hu}, \mathrm{Y}$; Ribbe, M.W. Decoding the nitrogenase mechanism: The homologue approach. Acc. Chem. Res. 2010, 43, 475-484.

7. Hellman, A.; Baerends, E.J.; Biczysko, M.; Bligaard, T.; Christensen, C.H.; Clary, D.C.; Dahl, S.; van Harrevelt, R.; Honkala, K.; Jonsson, H.; et al. Predicting catalysis: Understanding ammonia synthesis from first-principles calculations. J. Phys. Chem. B 2006, 110, 17719-17735.

8. Schrock, R.R. Reduction of dinitrogen. Proc. Natl. Acad. Sci. USA 2006, 103, 17087.

9. Smil, V. Enriching the Earth: Fritz Haber, Carl Bosch and the Transformation of World Food Production; Massachusetts Institute of Technology Press: Cambridge, MA, USA, 2001.

10. Erisman, J.W.; Sutton, M.A.; Galloway, J.; Klimont, Z.; Winiwarter, W. How a century of ammonia synthesis changed the world. Nat. GeoSci. 2008, 1, 636-639.

11. U.S. Geographical Survey, Mineral Commodity Surveys; U.S. Department of the Interior: Washington, DC, USA, 2015.

12. Corbridge, D. Phosphorus: An Outline of its Chemistry, Biochemistry, and Technology, 5th ed.; Elsevier: New York, NY, USA, 1994.

13. Quin, L.D. A Guide to Organophosphorus Chemistry; Wiley: New York, NY, USA, 2000.

14. Engel, R. Synthesis of Carbon Phosphorus Bonds, 2nd ed.; CRC Press: Boca Raton, FL, USA, 2004.

15. Withers, P.J.A.; Elser, J.J.; Hilton, J.; Ohtake, H.; Schipper, W.J.; van Dijk, K.C. Greening the global phosphorus cycle: How green chemistry can help achieve planetary $\mathrm{P}$ sustainability. Green Chem. 2015, 17, 2087-2099.

16. Liu, H. Catalytic Ammonia Synthesis; Plenum Press: New York, NY, USA, 1991.

17. Schlögl, R. Catalytic synthesis of ammonia-A "Never-Ending Story"? Angew. Chem. Int. Ed. 2003, 42, 2004-2008.

18. Leigh, G.J. Haber-Bosch and Other Industrial Processes. In Catalysts for Nitrogen Fixation; Smith, B., Richards, R., Newton, W., Eds.; Springer: Houten, The Netherlands, 2004; Volume 1, pp. 33-54.

19. Studt, F.; Tuczek, F. Theoretical, spectroscopic, and mechanistic studies on transition-metal dinitrogen complexes: Implications to reactivity and relevance to the nitrogenase problem. J. Comput. Chem. 2006, 27, 1278-1291.

20. MacKay, B.A.; Fryzuk, M.D. Dinitrogen coordination chemistry: On the biomimetic borderlands. Chem. Rev. 2004, 104, 385-401.

21. Barrière, F. Model Complexes of the Active Site of Nitrogenases: Recent Advances. In Bioinspired Catalysis; Wiley-VCH Verlag GmbH \& Co. KGaA: Weinheim, Germany, 2014; pp. 225-248. 
22. Rolff, M.; Tuczek, F. Nitrogenase and Nitrogen Activation. In Comprehensive Inorganic Chemistry II, 2nd ed.; Poeppelmeier, J.R., Ed.; Elsevier: Amsterdam, The Netherlands, 2013; pp. 593-618.

23. Ribbe, M.W. Nitrogen Fixation: Methods and Protocols; Humana Press: New York, NY, USA, 2011.

24. Barrière, F. Modeling of the molybdenum center in the nitrogenase FeMo-cofactor. Coord. Chem. Rev. 2003, 236, 71-89.

25. Smith, B.E.; Durrant, M.C.; Fairhurst, S.A.; Gormal, C.A.; Grönberg, K.L.C.; Henderson, R.A.; Ibrahim, S.K.; le Gall, T.; Pickett, C.J. Exploring the reactivity of the isolated iron-molybdenum cofactor of nitrogenase. Coord. Chem. Rev. 1999, 185-186, 669-687.

26. Rehder, D. Vanadium nitrogenase. J. Inorg. Biochem. 2000, 80, 133-136.

27. MacLachlan, E.A.; Fryzuk, M.D. Synthesis and reactivity of side-on-bound dinitrogen metal complexes. Organometallics 2006, 25, 1530-1543.

28. Fryzuk, M.D. Side-on end-on bound dinitrogen: An activated bonding mode that facilitates functionalizing molecular nitrogen. Acc. Chem. Res. 2009, 42, 127-133.

29. Poveda, A.; Perilla, I.C.; Pérez, C.R. Some considerations about coordination compounds with end-on dinitrogen. J. Coord. Chem. 2001, 54, 427-440.

30. Gambarotta, S.; Scott, J. Multimetallic cooperative activation of $\mathrm{N}_{2}$. Angew. Chem. Int. Ed. 2004, 43, 5298-5308.

31. McWilliams, S.F.; Holland, P.L. Dinitrogen binding and cleavage by multinuclear iron complexes. Acc. Chem. Res. 2015, 48, 2059-2065.

32. Ballmann, J.; Munha, R.F.; Fryzuk, M.D. The hydride route to the preparation of dinitrogen complexes. Chem. Commun. 2010, 46, 1013-1025.

33. Jia, H.-P.; Quadrelli, E.A. Mechanistic aspects of dinitrogen cleavage and hydrogenation to produce ammonia in catalysis and organometallic chemistry: Relevance of metal hydride bonds and dihydrogen. Chem. Soc. Rev. 2014, 43, 547-564.

34. Sivasankar, C.; Baskaran, S.; Tamizmani, M.; Ramakrishna, K. Lessons learned and lessons to be learned for developing homogeneous transition metal complexes catalyzed reduction of $\mathrm{N}_{2}$ to ammonia. J. Organomet. Chem. 2014, 752, 44-58.

35. Tanabe, Y.; Nishibayashi, Y. Developing more sustainable processes for ammonia synthesis. Coord. Chem. Rev. 2013, 257, 2551-2564.

36. Van der Ham, C.J.M.; Koper, M.T.M.; Hetterscheid, D.G.H. Challenges in reduction of dinitrogen by proton and electron transfer. Chem. Soc. Rev. 2014, 43, 5183-5191.

37. Rebreyend, C.; de Bruin, B. Photolytic $\mathrm{N}_{2}$ splitting: A road to sustainable $\mathrm{NH}_{3}$ production? Angew. Chem. Int. Ed. 2015, 54, 42-44.

38. Himmel, H.-J.; Reiher, M. Intrinsic dinitrogen activation at bare metal atoms. Angew. Chem. Int. Ed. 2006, 45, 6264-6288.

39. Chow, C.; Taoufik, M.; Quadrelli, E.A. Ammonia and dinitrogen activation by surface organometallic chemistry on silica-grafted tantalum hydrides. Eur. J. Inorg. Chem. 2011, 2011, 1349-1359.

40. Ohki, Y.; Fryzuk, M.D. Dinitrogen activation by group 4 metal complexes. Angew. Chem. Int. Ed. 2007, 46, 3180-3183. 
41. Chirik, P.J. Dinitrogen functionalization with bis(cyclopentadienyl) complexes of zirconium and hafnium. Dalton Trans. 2007, 1, 16-25.

42. Kuganathan, N.; Green, J.C.; Himmel, H.-J. Dinitrogen fixation and activation by Ti and $\mathrm{Zr}$ atoms, clusters and complexes. New J. Chem. 2006, 30, 1253-1262.

43. Crossland, J.L.; Tyler, D.R. Iron-dinitrogen coordination chemistry: Dinitrogen activation and reactivity. Coord. Chem. Rev. 2010, 254, 1883-1894.

44. Hazari, N. Homogeneous iron complexes for the conversion of dinitrogen into ammonia and hydrazine. Chem. Soc. Rev. 2010, 39, 4044-4056.

45. Schrock, R.R. Catalytic reduction of dinitrogen to ammonia at a single molybdenum center. Acc. Chem. Res. 2005, 38, 955-962.

46. Nishibayashi, Y. Molybdenum-catalyzed reduction of molecular dinitrogen into ammonia under ambient reaction conditions. Comptes Rendus Chim. 2015, 18, 776-784.

47. Schrock, R.R. Catalytic Reduction of Dinitrogen to Ammonia by Molybdenum. In Catalysis without Precious Metals; Wiley-VCH Verlag GmbH \& Co. KGaA: Weinheim, Germany, 2010; pp. 25-50.

48. Khoenkhoen, N.; de Bruin, B.; Reek, J.N.H.; Dzik, W.I. Reactivity of dinitrogen bound to mid- and late-transition-metal centers. Eur. J. Inorg. Chem. 2015, 2015, 567-598.

49. Evans, W.J.; Lee, D.S. Early developments in lanthanide-based dinitrogen reduction chemistry. Can. J. Chem. 2005, 83, 375-384.

50. Gardiner, M.G.; Stringer, D.N. Dinitrogen and related chemistry of the lanthanides: A review of the reductive capture of dinitrogen, as well as mono- and di-aza containing ligand chemistry of relevance to known and postulated metal mediated dinitrogen derivatives. Materials 2010, 3, $841-862$.

51. Gardner, B.M.; Liddle, S.T. Small-molecule activation at uranium(III). Eur. J. Inorg. Chem. 2013, 2013, 3753-3770.

52. Liddle, S.T. The renaissance of non-aqueous uranium chemistry. Angew. Chem. Int. Ed. 2015, $54,8604-8641$.

53. Scherer, O.J. Complexes with substituent-free acyclic and cyclic phosphorus, arsenic, antimony, and bismuth ligands. Angew. Chem. Int. Ed. 1990, 29, 1104-1122.

54. Scherer, O.J. $\mathrm{P}_{n}$ and As $n$ ligands: A novel chapter in the chemistry of phosphorus and arsenic. Acc. Chem. Res. 1999, 32, 751-762.

55. Peruzzini, M.; Gonsalvi, L.; Romerosa, A. Coordination chemistry and functionalization of white phosphorus via transition metal complexes. Chem. Soc. Rev. 2005, 34, 1038-1047.

56. Vaira, M.D.; Sacconi, L. Transition metal complexes with cyclo-triphosphorus $\left(\eta^{3}-\mathrm{P}_{3}\right)$ and tetrahedro-tetraphosphorus $\left(\eta^{1}-\mathrm{P}_{4}\right)$ ligands. Angew. Chem. Int. Ed. 1982, 21, 330-342.

57. Figueroa, J.S.; Cummins, C.C. A niobaziridine hydride system for white phosphorus or dinitrogen activation and N- or P-atom transfer. Dalton Trans. 2006, 2161-2168.

58. Cossairt, B.M.; Piro, N.A.; Cummins, C.C. Early-transition-metal-mediated activation and transformation of white phosphorus. Chem. Rev. 2010, 110, 4164-4177.

59. Caporali, M.; Gonsalvi, L.; Rossin, A.; Peruzzini, M. P4 activation by late-transition metal complexes. Chem. Rev. 2010, 110, 4178-4235. 
60. Giffin, N.A.; Masuda, J.D. Reactivity of white phosphorus with compounds of the p-block. Coord. Chem. Rev. 2011, 255, 1342-1359.

61. Balázs, G.; Seitz, A.; Scheer, M. Activation of White Phosphorus ( $\left.\mathrm{P}_{4}\right)$ by Main Group Elements and Compounds. In Comprehensive Inorganic Chemistry II, 2nd ed.; Poeppelmeier, J.R., Ed.; Elsevier: Amsterdam, The Netherlands, 2013; pp. 1105-1132.

62. Khan, S.; Sen, S.S.; Roesky, H.W. Activation of phosphorus by group 14 elements in low oxidation states. Chem. Commun. 2012, 48, 2169-2179.

63. Johnson, B.P.; Balázs, G.; Scheer, M. Low-coordinate E1 ligand complexes of group 15 elements-A developing area. Coord. Chem. Rev. 2006, 250, 1178-1195.

64. Scheer, M.; Balázs, G.; Seitz, A. P4 activation by main group elements and compounds. Chem. Rev. 2010, 110, 4236-4256.

65. Fang, M.; Bates, J.E.; Lorenz, S.E.; Lee, D.S.; Rego, D.B.; Ziller, J.W.; Furche, F.; Evans, W.J. $\left(\mathrm{N}_{2}\right)^{3-}$ radical chemistry via trivalent lanthanide salt/alkali metal reduction of dinitrogen: New syntheses and examples of $\left(\mathrm{N}_{2}\right)^{2-}$ and $\left(\mathrm{N}_{2}\right)^{3-}$ complexes and density functional theory comparisons of closed shell $\mathrm{Sc}^{3+}, \mathrm{Y}^{3+}$, and $\mathrm{Lu}^{3+}$ versus $4 \mathrm{f}^{9} \mathrm{Dy}^{3+}$. Inorg. Chem. 2011, 50, 1459-1469.

66. Fryzuk, M.D.; Johnson, S.A. The continuing story of dinitrogen activation. Coord. Chem. Rev. 2000, 200-202, 379-409.

67. Jaroschik, F.; Momin, A.; Nief, F.; LeGoff, X.-F.; Deacon, G.B.; Junk, P.C. Dinitrogen reduction and $\mathrm{C}-\mathrm{H}$ activation by the divalent organoneodymium complex $\left[\left(\mathrm{C}_{5} \mathrm{H}_{2}{ }^{t} \mathrm{Bu}_{3}\right)_{2} \mathrm{Nd}(\mu-\right.$ I)K([18]crown-6)]. Angew. Chem. Int. Ed. 2009, 48, 1117-1121.

68. Evans, W.J.; Rego, D.B.; Ziller, J.W. Synthesis, structure, and ${ }^{15}$ N-NMR studies of paramagnetic lanthanide complexes obtained by reduction of dinitrogen. Inorg. Chem. 2006, 45, 10790-10798.

69. Demir, S.; Lorenz, S.E.; Fang, M.; Furche, F.; Meyer, G.; Ziller, J.W.; Evans, W.J. Synthesis, structure, and density functional theory analysis of a scandium dinitrogen complex, $\left[\left(\mathrm{C}_{5} \mathrm{Me}_{4} \mathrm{H}\right)_{2} \mathrm{Sc}\right]_{2}\left(\mu-\eta^{2}: \eta^{2}-\mathrm{N}_{2}\right)$. J. Am. Chem. Soc. 2010, 132, 11151-11158.

70. Demir, S.; Siladke, N.A.; Ziller, J.W.; Evans, W.J. Scandium and yttrium metallocene borohydride complexes: Comparisons of $\left(\mathrm{BH}_{4}\right)^{1-}$ vs. $\left(\mathrm{BPh}_{4}\right)^{1-}$ coordination and reactivity. Dalton Trans. 2012, 41, 9659-9666.

71. Schmiege, B.M.; Ziller, J.W.; Evans, W.J. Reduction of dinitrogen with an yttrium metallocene hydride precursor, [(C5 $\left.\left.\mathrm{Ce}_{5}\right)_{2} \mathrm{YH}\right]_{2}$. Inorg. Chem. 2010, 49, 10506-10511.

72. Evans, W.J.; Ulibarri, T.A.; Ziller, J.W. Isolation and X-ray crystal structure of the first dinitrogen complex of an f-element metal, $\left[\left(\mathrm{C}_{5} \mathrm{Me}_{5}\right)_{2} \mathrm{Sm}\right]_{2} \mathrm{~N}_{2}$. J. Am. Chem. Soc. 1988, 110, 6877-6879.

73. Fieser, M.E.; Bates, J.E.; Ziller, J.W.; Furche, F.; Evans, W.J. Dinitrogen reduction via photochemical activation of heteroleptic tris(cyclopentadienyl) rare-earth complexes. $\mathrm{J}$. Am. Chem. Soc. 2013, 135, 3804-3807.

74. Evans, W.J.; Allen, N.T.; Ziller, J.W. Expanding divalent organolanthanide chemistry: The first organothulium(II) complex and the in situ organodysprosium(II) reduction of dinitrogen. Angew. Chem. Int. Ed. 2002, 41, 359-361.

75. Evans, W.J.; Allen, N.T.; Ziller, J.W. Facile dinitrogen reduction via organometallic Tm(II) chemistry. J. Am. Chem. Soc. 2001, 123, 7927-7928. 
76. Mueller, T.J.; Fieser, M.E.; Ziller, J.W.; Evans, W.J. $\left(\mathrm{C}_{5} \mathrm{Me}_{4} \mathrm{H}\right)^{1-}$-based reduction of dinitrogen by the mixed ligand tris(polyalkylcyclopentadienyl) lutetium and yttrium complexes, $\left(\mathrm{C}_{5} \mathrm{Me}_{5}\right)_{3-x}\left(\mathrm{C}_{5} \mathrm{Me}_{4} \mathrm{H}\right)_{x}$ Ln. Chem. Sci. 2011, 2, 1992-1996.

77. Lorenz, S.E.; Schmiege, B.M.; Lee, D.S.; Ziller, J.W.; Evans, W.J. Synthesis and reactivity of bis(tetramethylcyclopentadienyl) yttrium metallocenes including the reduction of $\mathrm{Me}_{3} \mathrm{SiN}_{3}$ to $\left[\left(\mathrm{Me}_{3} \mathrm{Si}\right)_{2} \mathrm{~N}\right]^{-}$with $\left[\left(\mathrm{C}_{5} \mathrm{Me}_{4} \mathrm{H}\right)_{2} \mathrm{Y}(\mathrm{THF})\right]_{2}\left(\mu-\eta^{2}: \eta^{2}-\mathrm{N}_{2}\right)$. Inorg. Chem. 2010, 49, 6655-6663.

78. MacDonald, M.R.; Ziller, J.W.; Evans, W.J. Synthesis of a crystalline molecular complex of $\mathrm{Y}^{2+}$, [(18-crown-6)K][(C5 $\left.\left.\mathrm{H}_{4} \mathrm{SiMe}_{3}\right)_{3} \mathrm{Y}\right]$. J. Am. Chem. Soc. 2011, 133, 15914-15917.

79. Evans, W.J.; Lee, D.S.; Lie, C.; Ziller, J.W. Expanding the LnZ 3 /alkali-metal reduction system to organometallic and heteroleptic precursors: Formation of dinitrogen derivatives of lanthanum. Angew. Chem. Int. Ed. 2004, 43, 5517-5519.

80. Evans, W.J.; Lee, D.S.; Johnston, M.A.; Ziller, J.W. The elusive $\left(\mathrm{C}_{5} \mathrm{Me}_{4} \mathrm{H}\right)_{3} \mathrm{Lu}$ : Its synthesis and $\mathrm{LnZ}_{3} / \mathrm{K} / \mathrm{N}_{2}$ reactivity. Organometallics 2005, 24, 6393-6397.

81. Evans, W.J.; Lee, D.S.; Rego, D.B.; Perotti, J.M.; Kozimor, S.A.; Moore, E.K.; Ziller, J.W. Expanding dinitrogen reduction chemistry to trivalent lanthanides via the LnZ $3 /$ Alkali metal reduction system: Evaluation of the generality of forming $\operatorname{Ln}_{2}\left(\mu-\eta^{2}: \eta^{2}-N_{2}\right)$ complexes via $\mathrm{LnZ}_{3} / \mathrm{K}$. J. Am. Chem. Soc. 2004, 126, 14574-14582.

82. Evans, W.J.; Zucchi, G.; Ziller, J.W. Dinitrogen reduction by Tm(II), Dy(II), and Nd(II) with simple amide and aryloxide ligands. J. Am. Chem. Soc. 2003, 125, 10-11.

83. Evans, W.J.; Lee, D.S.; Ziller, J.W. Reduction of dinitrogen to planar bimetallic $\mathrm{M}_{2}\left(\mu-\eta^{2}: \eta^{2}-\mathrm{N}_{2}\right)$ complexes of $\mathrm{Y}, \mathrm{Ho}, \mathrm{Tm}$, and $\mathrm{Lu}$ using the $\mathrm{K} / \mathrm{Ln}\left[\mathrm{N}\left(\mathrm{SiMe}_{3}\right)_{2}\right]_{3}$ reduction system. J. Am. Chem. Soc. 2004, 126, 454-455.

84. Corbey, J.F.; Farnaby, J.H.; Bates, J.E.; Ziller, J.W.; Furche, F.; Evans, W.J. Varying the Lewis base coordination of the $\mathrm{Y}_{2} \mathrm{~N}_{2}$ core in the reduced dinitrogen complexes $\left\{\left[\left(\mathrm{Me} \mathrm{Si}_{2} \mathrm{~N}\right]_{2}(\mathrm{~L}) \mathrm{Y}\right\}_{2}\right.$ $\left(\mu-\eta^{2}: \eta^{2}-\mathrm{N}_{2}\right) \quad(\mathrm{L}=$ benzonitrile, pyridines, triphenylphosphine oxide, and trimethylamine N-oxide). Inorg. Chem. 2012, 51, 7867-7874.

85. Campazzi, E.; Solari, E.; Floriani, C.; Scopelliti, R. The fixation and reduction of dinitrogen using lanthanides: Praseodymium and neodymium meso-octaethylporphyrinogen-dinitrogen complexes. Chem. Commun. 1998, 2603-2604.

86. Cheng, J.; Takats, J.; Ferguson, M.J.; McDonald, R. Heteroleptic Tm(II) complexes: One more success for Trofimenko's scorpionates. J. Am. Chem. Soc. 2008, 130, 1544-1545.

87. Mansell, S.M.; Farnaby, J.H.; Germeroth, A.I.; Arnold, P.L. Thermally stable uranium dinitrogen complex with siloxide supporting ligands. Organometallics 2013, 32, 4214-4222.

88. Mansell, S.M.; Kaltsoyannis, N.; Arnold, P.L. Small molecule activation by uranium tris(aryloxides): Experimental and computational studies of binding of $\mathrm{N}_{2}$, coupling of $\mathrm{CO}$, and deoxygenation insertion of $\mathrm{CO}_{2}$ under ambient conditions. J. Am. Chem. Soc. 2011, 133, 9036-9051.

89. Perrin, L.; Maron, L.; Eisenstein, O.; Schwartz, D.J.; Burns, C.J.; Andersen, R.A. Bonding of H2, $\mathrm{N}_{2}$, ethylene, and acetylene to bivalent lanthanide metallocenes: Trends from DFT calculations on $\mathrm{Cp}_{2} \mathrm{M}$ and $\mathrm{Cp}_{2}{ }_{2} \mathrm{M}(\mathrm{M}=\mathrm{Sm}, \mathrm{Eu}, \mathrm{Yb})$ and experiments with $\mathrm{Cp}_{2}{ }_{2} \mathrm{Yb}$. Organometallics 2003, 22, 5447-5453. 
90. Hamaed, H.; Lo, A.Y.; Lee, D.S.; Evans, W.J.; Schurko, R.W. Solid-state ${ }^{139}$ La- and ${ }^{15}$ N-NMR spectroscopy of lanthanum-containing metallocenes. J. Am. Chem. Soc. 2006, 128, 12638-12639.

91. Evans, W.J.; Davis, B.L. Chemistry of tris(pentamethylcyclopentadienyl) f-element complexes, (C5 $\left.\mathrm{Me}_{5}\right)_{3} \mathrm{M}$. Chem. Rev. 2002, 102, 2119-2136.

92. Fieser, M.E.; Johnson, C.W.; Bates, J.E.; Ziller, J.W.; Furche, F.; Evans, W.J. Dinitrogen reduction, sulfur reduction, and isoprene polymerization via photochemical activation of trivalent bis(cyclopentadienyl) rare-earth-metal allyl complexes. Organometallics 2015, 34, 4387-4393.

93. Evans, W.J.; Fang, M.; Zucchi, G.; Furche, F.; Ziller, J.W.; Hoekstra, R.M.; Zink, J.I. Isolation of dysprosium and yttrium complexes of a three-electron reduction product in the activation of dinitrogen, the $\left(\mathrm{N}_{2}\right)^{3-}$ radical. J. Am. Chem. Soc. 2009, 131, 11195-11202.

94. Rinehart, J.D.; Fang, M.; Evans, W.J.; Long, J.R. Strong exchange and magnetic blocking in $\mathrm{N}_{2}{ }^{3-}$-radical-bridged lanthanide complexes. Nat. Chem. 2011, 3, 538-542.

95. Rinehart, J.D.; Fang, M.; Evans, W.J.; Long, J.R. A $\mathrm{N}_{2}{ }^{3-}$ radical-bridged terbium complex exhibiting magnetic hysteresis at 14 K. J. Am. Chem. Soc. 2011, 133, 14236-14239.

96. Roy, L.E.; Hughbanks, T. Magnetic coupling in dinuclear Gd complexes. J. Am. Chem. Soc. 2006, 128, 568-575.

97. Meihaus, K.R.; Corbey, J.F.; Fang, M.; Ziller, J.W.; Long, J.R.; Evans, W.J. Influence of an inner-sphere $\mathrm{K}^{+}$ion on the magnetic behavior of $\mathrm{N}_{2}{ }^{3-}$ radical-bridged dilanthanide complexes isolated using an external magnetic field. Inorg. Chem. 2014, 53, 3099-3107.

98. Allen, F.H.; Kennard, O.; Watson, D.G.; Brammer, L.; Orpen, A.G.; Taylor, R. Tables of bond lengths determined by X-ray and neutron diffraction. Part 1. Bond lengths in organic compounds. J. Chem. Soc. Perkin Trans. 1987, 2, S1-S19.

99. Zhang, Y.-Q.; Luo, C.-L.; Wang, B.-W.; Gao, S. Understanding the magnetic anisotropy in a family of $\mathrm{N}_{2}{ }^{3-}$ radical-bridged lanthanide complexes: Density functional theory and ab initio calculations. J. Phys. Chem. A 2013, 117, 10873-10880.

100. Rajeshkumar, T.; Rajaraman, G. Is a radical bridge a route to strong exchange interactions in lanthanide complexes? A computational examination. Chem. Commun. 2012, 48, 7856-7858.

101. Jubb, J.; Gambarotta, S. Dinitrogen reduction operated by a samarium macrocyclic complex. Encapsulation of dinitrogen into a $\mathrm{Sm}_{2} \mathrm{Li}_{4}$ metallic cage. J. Am. Chem. Soc. 1994, 116, 4477-4478.

102. Dubé, T.; Conoci, S.; Gambarotta, S.; Yap, G.P.A.; Vasapollo, G. Tetrametallic reduction of dinitrogen: Formation of a tetranuclear samarium dinitrogen complex. Angew. Chem. Int. Ed. 1999, 38, 3657-3659.

103. Dubé, T.; Ganesan, M.; Conoci, S.; Gambarotta, S.; Yap, G.P.A. Tetrametallic divalent samarium cluster hydride and dinitrogen complexes. Organometallics 2000, 19, 3716-3721.

104. Bérubé, C.D.; Yazdanbakhsh, M.; Gambarotta, S.; Yap, G.P.A. Serendipitous isolation of the first example of a mixed-valence samarium tripyrrole complex. Organometallics 2003, 22, 3742-3747.

105. Guan, J.; Dubé, T.; Gambarotta, S.; Yap, G.P.A. Dinitrogen labile coordination versus fourelectron reduction, THF cleavage, and fragmentation promoted by a (calix-tetrapyrrole)Sm(II) complex. Organometallics 2000, 19, 4820-4827. 
106. Ganesan, M.; Gambarotta, S.; Yap, G.P.A. Highly reactive $\mathrm{Sm}^{\mathrm{II}}$ macrocyclic clusters: Precursors to $\mathrm{N}_{2}$ reduction. Angew. Chem. Int. Ed. 2001, 40, 766-769.

107. Fox, A.R.; Bart, S.C.; Meyer, K.; Cummins, C.C. Towards uranium catalysts. Nature 2008, 455, 341-349.

108. Huang, Q.-R.; Kingham, J.R.; Kaltsoyannis, N. The strength of actinide-element bonds from the quantum theory of atoms-in-molecules. Dalton Trans. 2015, 44, 2554-2566.

109. Roussel, P.; Scott, P. Complex of dinitrogen with trivalent uranium. J. Am. Chem. Soc. 1998, 120, 1070-1071.

110. Odom, A.L.; Arnold, P.L.; Cummins, C.C. Heterodinuclear uranium/molybdenum dinitrogen complexes. J. Am. Chem. Soc. 1998, 120, 5836-5837.

111. Cloke, F.G.N.; Hitchcock, P.B. Reversible binding and reduction of dinitrogen by a uranium(III) pentalene complex. J. Am. Chem. Soc. 2002, 124, 9352-9353.

112. Evans, W.J.; Kozimor, S.A.; Ziller, J.W. A monometallic f element complex of dinitrogen: $\left(\mathrm{C}_{5} \mathrm{Me}_{5}\right)_{3} \mathrm{U}\left(\eta^{1}-\mathrm{N}_{2}\right)$. J. Am. Chem. Soc. 2003, 125, 14264-14265.

113. Laplaza, C.E.; Cummins, C.C. Dinitrogen cleavage by a three-coordinate molybdenum(III) complex. Science 1995, 268, 861-863.

114. Mindiola, D.J.; Meyer, K.; Cherry, J.-P.F.; Baker, T.A.; Cummins, C.C. Dinitrogen cleavage stemming from a heterodinuclear niobium/molybdenum $\mathrm{N}_{2}$ complex: New nitridoniobium systems including a niobazene cyclic trimer. Organometallics 2000, 19, 1622-1624.

115. Curley, J.J.; Cook, T.R.; Reece, S.Y.; Müller, P.; Cummins, C.C. Shining light on dinitrogen cleavage: Structural features, redox chemistry, and photochemistry of the key intermediate bridging dinitrogen complex. J. Am. Chem. Soc. 2008, 130, 9394-9405.

116. Kaltsoyannis, N.; Scott, P. Evidence for actinide metal to ligand $\pi$ backbonding. Density functional investigations of the electronic structure of $\left[\left\{\left(\mathrm{NH}_{2}\right)_{3}\left(\mathrm{NH}_{3}\right) \mathrm{U}_{2}\left(\mu^{2}-\eta^{2}: \eta^{2}-\mathrm{N}_{2}\right)\right]\right.$. Chem. Commun. 1998, 1665-1666.

117. Roussel, P.; Errington, W.; Kaltsoyannis, N.; Scott, P. Back bonding without $\sigma$-bonding: A unique $\pi$-complex of dinitrogen with uranium. J. Organomet. Chem. 2001, 635, 69-74.

118. Cloke, F.G.N.; Green, J.C.; Kaltsoyannis, N. Electronic structure of $\left[\mathrm{U}_{2}\left(\mu^{2}-\mathrm{N}_{2}\right)\left(\eta^{5}-\mathrm{C}_{5} \mathrm{Me}_{5}\right)_{2}\right.$ $\left.\left(\eta^{8}-\mathrm{C}_{8} \mathrm{H}_{4}\left(\mathrm{SiPr}_{3}{ }_{3}\right)_{2}\right)_{2}\right]$. Organometallics 2004, 23, 832-835.

119. Korobkov, I.; Gambarotta, S.; Yap, G.P.A. A highly reactive uranium complex supported by the calix[4]tetrapyrrole tetraanion affording dinitrogen cleavage, solvent deoxygenation, and polysilanol depolymerization. Angew. Chem. Int. Ed. 2002, 41, 3433-3436.

120. Korobkov, I.; Gambarotta, S.; Yap, G.P.A. Amide from dinitrogen by in situ cleavage and partial hydrogenation promoted by a transient zero-valent thorium synthon. Angew. Chem. Int. Ed. 2003, 42, 4958-4961.

121. Corbridge, D.E.C.; Lowe, E.J. Structure of white phosphorus: Single crystal X-ray examination. Nature 1952, 170, 629-629.

122. Kühl, O. Phosphorus-31 NMR Spectroscopy: A Concise Introduction for the Synthetic Organic and Organometallic Chemist; Springer-Verlag GmbH: Berlin, Germany; Heidelberg, Germany, 2008.

123. Konchenko, S.N.; Pushkarevsky, N.A.; Gamer, M.T.; Köppe, R.; Schnöckel, H.; Roesky, P.W. $\left[\left\{\left(\eta^{5}-\mathrm{C}_{5} \mathrm{Me}_{5}\right)_{2} \mathrm{Sm}\right\}_{4} \mathrm{P}_{8}\right]:$ A molecular polyphosphide of the rare-earth elements. J. Am. Chem. Soc. 2009, 131, 5740-5741. 
124. Huang, W.; Diaconescu, P.L. P4 activation by group 3 metal arene complexes. Chem. Commun. 2012, 48, 2216-2218.

125. Huang, W.; Diaconescu, P.L. P4 Activation by lanthanum and lutetium naphthalene complexes supported by a ferrocene diamide ligand. Eur. J. Inorg. Chem. 2013, 2013, 4090-4096.

126. Turbervill, R.S.P.; Goicoechea, J.M. From clusters to unorthodox pnictogen sources: Solutionphase reactivity of $\left[\mathrm{E}_{7}\right]^{3-}(\mathrm{E}=\mathrm{P}-\mathrm{Sb})$ anions. Chem. Rev. 2014, 114, 10807-10828.

127. Shannon, R. Revised effective ionic radii and systematic studies of interatomic distances in halides and chalcogenides. Acta Crystallogr. Sect. A Found. Crystallogr. 1976, 32, 751-767.

128. Scherer, O.J.; Werner, B.; Heckmann, G.; Wolmershäuser, G. Bicyclic $\mathrm{P}_{6}$ as complex ligand. Angew. Chem. Int. Ed. 1991, 30, 553-555.

129. Stephens, F.H. Activation of White Phosphorus by Molybdenum and Uranium tris-Amides. Ph.D. Thesis, Massachusetts Institute of Technology, Cambridge, MA, USA, May 2004.

130. Frey, A.S.P.; Cloke, F.G.N.; Hitchcock, P.B.; Green, J.C. Activation of $\mathrm{P}_{4}$ by $\mathrm{U}\left(\eta^{5}-\mathrm{C}_{5} \mathrm{Me}_{5}\right)$ $\left(\eta^{8}-\mathrm{C}_{8} \mathrm{H}_{6}\left(\mathrm{Si}^{i} \mathrm{Pr}_{3}\right)_{2}-1,4\right)(\mathrm{THF})$; the X-ray structure of $\left[\mathrm{U}\left(\eta^{5}-\mathrm{C}_{5} \mathrm{Me}_{5}\right)\left(\eta^{8}-\mathrm{C}_{8} \mathrm{H}_{6}\left(\mathrm{Si}^{i} \mathrm{Pr}_{3}\right)_{2}-1,4\right)\right]_{2}(\mu-$ $\left.\eta^{2}: \eta^{2}-\mathrm{P}_{4}\right)$. New J. Chem. 2011, 35, 2022-2026.

131. Gardner, B.M.; Tuna, F.; McInnes, E.J.L.; McMaster, J.; Lewis, W.; Blake, A.J.; Liddle, S.T. An inverted-sandwich diuranium $\mu-\eta^{5}: \eta^{5}$-cyclo- $\mathrm{P}_{5}$ complex supported by $\mathrm{U}_{-} \mathrm{P}_{5} \delta$-bonding. Angew. Chem. Int. Ed. 2015, 54, 7068-7072.

132. Forfar, L.C.; Clark, T.J.; Green, M.; Mansell, S.M.; Russell, C.A.; Sanguramath, R.A.; Slattery, J.M. White phosphorus as a ligand for the coinage metals. Chem. Commun. 2012, 48, 1970-1972.

133. Spitzer, F.; Sierka, M.; Latronico, M.; Mastrorilli, P.; Virovets, A.V.; Scheer, M. Fixation and release of intact $\mathrm{E}_{4}$ tetrahedra (E = P, As). Angew. Chem. Int. Ed. 2015, 54, 4392-4396.

134. Patel, D.; Tuna, F.; McInnes, E.J.L.; Lewis, W.; Blake, A.J.; Liddle, S.T. An actinide zintl cluster: A tris(triamidouranium) $\mu_{3}-\eta^{2}: \eta^{2}: \eta^{2}$-heptaphosphanortricyclane and its diverse synthetic utility. Angew. Chem. Int. Ed. 2013, 52, 13334-13337.

135. Laplaza, C.E.; Davis, W.M.; Cummins, C.C. A molybdenum-phosphorus triple bond: Synthesis, structure, and reactivity of the terminal phosphido $\left(\mathrm{P}^{3-}\right)$ complex $\left[\mathrm{Mo}(\mathrm{P})(\mathrm{NRAr})_{3}\right]$. Angew. Chem. Int. Ed. 1995, 34, 2042-2044.

136. Cherry, J.-P.F.; Stephens, F.H.; Johnson, M.J.A.; Diaconescu, P.L.; Cummins, C.C. Terminal phosphide and dinitrogen molybdenum compounds obtained from pnictide-bridged precursors. Inorg. Chem. 2001, 40, 6860-6862.

137. Chisholm, M.H.; Folting, K.; Pasterczyk, J.W. A phosphido-capped tritungsten alkoxide cluster: $\mathrm{W}_{3}\left(\mu_{3}-\mathrm{P}\right)\left(\mu-\mathrm{OCH}_{2}-t-\mathrm{Bu}\right)_{3}\left(\mathrm{OCH}_{2}-t-\mathrm{Bu}\right)_{6}$ and speculation upon the existence of a reactive $\left(t-\mathrm{BuCH}_{2} \mathrm{O}\right)_{3} \mathrm{~W} \equiv \mathrm{P}$ intermediate. Inorg. Chem. 1988, 27, 3057-3058.

138. Scherer, O.J.; Sitzmann, H.; Wolmershäuser, G. Umsetzung von $\mathrm{P}_{4}$ mit $\left(\eta^{5}-\mathrm{C}_{5} \mathrm{H}_{5}\right)(\mathrm{CO})_{2} \mathrm{Mo} \equiv \mathrm{Mo}$ $(\mathrm{CO})_{2}\left(\eta^{5}-\mathrm{C}_{5} \mathrm{H}_{5}\right)$ zu den tetraedrischen molybdänkomplexen $\mathrm{P}_{n}\left[\mathrm{Mo}(\mathrm{CO})_{2}\left(\eta^{5}-\mathrm{C}_{5} \mathrm{H}_{5}\right)\right]_{4-n}(n=2,3)$. J. Organomet. Chem. 1984, 268, C9-C12.

139. Di Vaira, M.; Ghilardi, C.A.; Midollini, S.; Sacconi, L. cyclo-Triphosphorus $\left(\delta-\mathrm{P}_{3}\right)$ as a ligand in cobalt and nickel complexes with 1,1,1-tris(diphenylphosphinomethyl)ethane. Formation and structures. J. Am. Chem. Soc. 1978, 100, 2550-2551.

140. Chirik, P.J.; Pool, J.A.; Lobkovsky, E. Functionalization of elemental phosphorus with $\left[\operatorname{Zr}\left(\eta^{5}-\right.\right.$ $\left.\left.\mathrm{C}_{5} \mathrm{Me}_{5}\right)\left(\eta^{5}-\mathrm{C}_{5} \mathrm{H}_{4}{ }^{t} \mathrm{Bu}\right) \mathrm{H}_{2}\right]_{2}$. Angew. Chem. Int. Ed. 2002, 41, 3463-3465. 
141. Gröer, T.; Baum, G.; Scheer, M. Complexes with a monohapto bound phosphorus tetrahedron and phosphaalkyne. Organometallics 1998, 17, 5916-5919.

142. Peruzzini, M.; Marvelli, L.; Romerosa, A.; Rossi, R.; Vizza, F.; Zanobini, F. Synthesis and characterisation of tetrahedro-tetraphosphorus complexes of rhenium-Evidence for the first bridging complex of white phosphorus. Eur. J. Inorg. Chem. 1999, 1999, 931-933.

143. Urnėžius, E.; Brennessel, W.W.; Cramer, C.J.; Ellis, J.E.; Schleyer, P.V.R. A carbon-free sandwich complex $\left[\left(\mathrm{P}_{5}\right)_{2} \mathrm{Ti}\right]^{2-}$. Science 2002, 295, 832-834.

144. Scherer, O.J.; Sitzmann, H.; Wolmershäuser, G. Hexaphosphabenzene as complex ligand. Angew. Chem. Int. Ed. 1985, 24, 351-353.

145. Scherer, O.J.; Berg, G.; Wolmershäuser, G. $\mathrm{P}_{8}$ and $\mathrm{P}_{12}$ as complex ligands. Chem. Ber. 1996, $129,53-58$.

146. Scheer, M.; Deng, S.; Scherer, O.J.; Sierka, M. Tetraphosphacyclopentadienyl and triphosphaallyl ligands in iron complexes. Angew. Chem. Int. Ed. 2005, 44, 3755-3758.

147. Scherer, O.J.; Schulze, J.; Wolmershäuser, G. Bicyclisches As6 als komplexligand. J. Organomet. Chem. 1994, 484, C5-C7.

148. Di Vaira, M.; Midollini, S.; Sacconi, L.; Zanobini, F. cyclo-Triarsenic as $\mu, \eta$-ligand in transition-metal complexes. Angew. Chem. Int. Ed. 1978, 17, 676-677.

149. Scherer, O.J.; Kemény, G.; Wolmershäuser, G. $\left[\mathrm{Cp}_{4} \mathrm{Fe}_{4}\left(\mathrm{E}_{2}\right)_{2}\right]$ clusters with triangulated dodecahedral $\mathrm{Fe}_{4} \mathrm{E}_{4}$ skeletons (E = P, As). Chem. Ber. 1995, 128, 1145-1148.

150. Schwarzmaier, C.; Timoshkin, A.Y.; Scheer, M. An end-on-coordinated As4 tetrahedron. Angew. Chem. Int. Ed. 2013, 52, 7600-7603.

151. Schwarzmaier, C.; Sierka, M.; Scheer, M. Intact As4 tetrahedra coordinated side-on to metal cations. Angew. Chem. Int. Ed. 2013, 52, 858-861.

152. Scherer, O.J.; Sitzmann, H.; Wolmershäuser, G. $\left(\mathrm{E}_{2}\right)_{2}$-einheiten $(\mathrm{E}=\mathrm{P}, \mathrm{As})$ als clusterbausteine. J. Organomet. Chem. 1986, 309, 77-86.

153. Spinney, H.A.; Piro, N.A.; Cummins, C.C. Triple-bond reactivity of an AsP complex intermediate: Synthesis stemming from molecular arsenic, As4. J. Am. Chem. Soc. 2009, 131, 16233-16243.

154. Heinl, S.; Scheer, M. Activation of group 15 based cage compounds by $\left[\mathrm{Cp}^{\mathrm{BIG}} \mathrm{Fe}(\mathrm{CO})_{2}\right]$ radicals. Chem. Sci. 2014, 5, 3221-3225.

155. Gra; Bodensteiner, M.; Zabel, M.; Scheer, M. Synthesis of arsenic-rich As $n$ ligand complexes from yellow arsenic. Chem. Sci. 2015, 6, 1379-1382.

156. Schwarzmaier, C.; Bodensteiner, M.; Timoshkin, A.Y.; Scheer, M. An approach to mixed $\mathrm{P}_{n} \mathrm{As}_{m}$ ligand complexes. Angew. Chem. Int. Ed. 2014, 53, 290-293.

157. Curley, J.J.; Piro, N.A.; Cummins, C.C. A terminal molybdenum arsenide complex synthesized from yellow arsenic. Inorg. Chem. 2009, 48, 9599-9601.

158. Balázs, G.; Sierka, M.; Scheer, M. Antimony-tungsten triple bond: A stable complex with a terminal antimony ligand. Angew. Chem. Int. Ed. 2005, 44, 4920-4924.

159. Evans, W.J.; Fang, M.; Bates, J.E.; Furche, F.; Ziller, J.W.; Kiesz, M.D.; Zink, J.I. Isolation of a radical dianion of nitrogen oxide $(\mathrm{NO})^{2-}$. Nat. Chem. 2010, 2, 644-647. 
160. Corbey, J.F.; Fang, M.; Ziller, J.W.; Evans, W.J. Cocrystallization of $\left(\mu-\mathrm{S}_{2}\right)^{2-}$ and $(\mu-\mathrm{S})^{2-}$ and formation of an $\left[\eta^{2}-\mathrm{S}_{3} \mathrm{~N}\left(\mathrm{SiMe}_{3}\right)_{2}\right]$ ligand from chalcogen reduction by $\left(\mathrm{N}_{2}\right)^{2-}$ in a bimetallic yttrium amide complex. Inorg. Chem. 2015, 54, 801-807.

161. Evans, W.J.; Lee, D.S.; Ziller, J.W.; Kaltsoyannis, N. Trivalent $\left[\left(\mathrm{C}_{5} \mathrm{Me}_{5}\right)_{2}(\mathrm{THF}) \mathrm{Ln}\right]_{2}\left(\mu-\eta^{2}: \eta^{2}-\mathrm{N}_{2}\right)$ complexes as reducing agents including the reductive homologation of $\mathrm{CO}$ to a ketene carboxylate, $\left(\mu-\eta^{4}-\mathrm{O}_{2} \mathrm{CCCO}\right)_{2}$. J. Am. Chem. Soc. 2006, 128, 14176-14184.

162. Lu, E.; Li, Y.; Chen, Y. A scandium terminal imido complex: Synthesis, structure and DFT studies. Chem. Commun. 2010, 46, 4469-4471.

163. Rong, W.; Cheng, J.; Mou, Z.; Xie, H.; Cui, D. Facile preparation of a scandium terminal imido complex supported by a phosphazene ligand. Organometallics 2013, 32, 5523-5529.

164. Schädle, D.; Meermann-Zimmermann, M.; Schädle, C.; Maichle-Mössmer, C.; Anwander, R. Rare-earth metal complexes with terminal imido ligands. Eur. J. Inorg. Chem. 2015, 2015, 1334-1339.

165. Sobota, P.; Janas, Z. Formation of a nitrogen-carbon bond from $\mathrm{N}_{2}$ and CO. Influence of $\mathrm{MgCl}_{2}$ on the $\mathrm{N}_{2}$ reduction process in the system $\mathrm{TiCl}_{4} / \mathrm{Mg}$. J. Organomet. Chem. 1984, 276, 171-176.

166. Semproni, S.P.; Margulieux, G.W.; Chirik, P.J. Di- and tetrametallic hafnocene oxamidides prepared from $\mathrm{CO}$-induced $\mathrm{N}_{2}$ bond cleavage and thermal rearrangement to hafnocene cyanide derivatives. Organometallics 2012, 31, 6278-6287.

167. Knobloch, D.J.; Lobkovsky, E.; Chirik, P.J. Carbon monoxide-induced dinitrogen cleavage with group 4 metallocenes: Reaction scope and coupling to $\mathrm{N}-\mathrm{H}$ bond formation and $\mathrm{CO}$ deoxygenation. J. Am. Chem. Soc. 2010, 132, 10553-10564.

168. Semproni, S.P.; Milsmann, C.; Chirik, P.J. Structure and reactivity of a hafnocene $\mu$-nitrido prepared from dinitrogen cleavage. Angew. Chem. Int. Ed. 2012, 51, 5213-5216.

169. Semproni, S.P.; Chirik, P.J. Synthesis of a base-free hafnium nitride from $\mathrm{N}_{2}$ cleavage: A versatile platform for dinitrogen functionalization. J. Am. Chem. Soc. 2013, 135, 11373-11383.

170. MacKay, B.A.; Johnson, S.A.; Patrick, B.O.; Fryzuk, M.D. Functionalization and cleavage of coordinated dinitrogen via hydroboration using primary and secondary boranes. Can. J. Chem. 2005, 83, 315-323.

171. Fryzuk, M.D.; MacKay, B.A.; Johnson, S.A.; Patrick, B.O. Hydroboration of coordinated dinitrogen: A new reaction for the $\mathrm{N}_{2}$ ligand that results in its functionalization and cleavage. Angew. Chem. Int. Ed. 2002, 41, 3709-3712.

172. Fryzuk, M.D.; MacKay, B.A.; Patrick, B.O. Hydrosilylation of a dinuclear tantalum dinitrogen complex: Cleavage of $\mathrm{N}_{2}$ and functionalization of both nitrogen atoms. J. Am. Chem. Soc. 2003, 125, 3234-3235.

173. Spencer, L.P.; MacKay, B.A.; Patrick, B.O.; Fryzuk, M.D. Inner-sphere two-electron reduction leads to cleavage and functionalization of coordinated dinitrogen. Proc. Natl. Acad. Sci. USA 2006, 103, 17094-17098.

174. Margulieux, G.W.; Turner, Z.R.; Chirik, P.J. Synthesis and ligand modification chemistry of a molybdenum dinitrogen complex: Redox and chemical activity of a bis(imino)pyridine ligand. Angew. Chem. Int. Ed. 2014, 53, 14211-14215. 
175. Milsmann, C.; Turner, Z.R.; Semproni, S.P.; Chirik, P.J. Azo N=N bond cleavage with a redox-active vanadium compound involving metal-ligand cooperativity. Angew. Chem. Int. Ed. 2012, 51, 5386-5390.

176. King, D.M.; Tuna, F.; McInnes, E.J.L.; McMaster, J.; Lewis, W.; Blake, A.J.; Liddle, S.T. Synthesis and structure of a terminal uranium nitride complex. Science 2012, 337, 717-720.

177. King, D.M.; Tuna, F.; McInnes, E.J.L.; McMaster, J.; Lewis, W.; Blake, A.J.; Liddle, S.T. Isolation and characterization of a uranium(VI)-nitride triple bond. Nat. Chem. 2013, 5, 482-488.

178. Cleaves, P.A.; King, D.M.; Kefalidis, C.E.; Maron, L.; Tuna, F.; McInnes, E.J.L.; McMaster, J.; Lewis, W.; Blake, A.J.; Liddle, S.T. Two-electron reductive carbonylation of terminal uranium(V) and uranium(VI) nitrides to cyanate by carbon monoxide. Angew. Chem. Int. Ed. 2014, 53, 10412-10415.

179. Gardner, B.M.; Balázs, G.; Scheer, M.; Tuna, F.; McInnes, E.J.L.; McMaster, J.; Lewis, W.; Blake, A.J.; Liddle, S.T. Triamidoamine uranium(IV)-arsenic complexes containing one-, two- and threefold U-As bonding interactions. Nat. Chem. 2015, 7, 582-590.

(C) 2015 by the authors; licensee MDPI, Basel, Switzerland. This article is an open access article distributed under the terms and conditions of the Creative Commons Attribution license (http://creativecommons.org/licenses/by/4.0/). 